T. Murai

Nagoya Math. J.

Vol. 125 (1992), 151-216

\title{
THE ARC-LENGTH VARIATION OF ANALYTIC CAPACITY AND A CONFORMAL GEOMETRY
}

\author{
TAKAFUMI MURAI
}

\section{§1. Introduction}

For a domain $\Omega$ in the extended complex plane $\mathbf{C} \cup\{\infty\}, H^{\infty}(\Omega)$ denotes the Banach space of bounded analytic functions in $\Omega$ with supremum norm $\|\cdot\|_{H^{\infty}}$. For $\zeta \in \Omega$, we put

$$
c(\zeta ; \Omega)=\sup \left\{\left|f^{\prime}(\zeta)\right| ; f \in H^{\infty}(\Omega),\|f\|_{H^{\infty}} \leq 1\right\},
$$

where $f^{\prime}(\infty)=\lim _{z \rightarrow \infty} z\{f(\infty)-f(z)\}$ if $\zeta=\infty$. The analytic capacity of a compact set $E$ in $\mathbf{C}$ is defined by

$$
\gamma(E)=c\left(\infty ; \Omega_{E}\right)
$$

where $\Omega_{E}$ is the component of $E^{c}=\mathbf{C} \cup\{\infty\}-E$ containing $\infty$. Ahlfors [1] shows that, for a domain $\Omega$ and a compact set $E \subset \Omega$, the equality $\gamma(E)=0$ holds if and only if any function in $H^{\infty}\left(\Omega \cap \Omega_{E}\right)$ has an analytic extension to $\Omega$. Thus a compact set $E$ satisfying Riemann's theorem of removable singularities is characterized by $r(E)=0$. Garabedian [8] studies analytic capacity from the point of view of the dual extremum problem. The quantitative properties of analytic capacity are important in the study of conformal mappings, the 2-dimensional fluid dynamics and singular integrals ([16], [17], [23]). Vitushkin [34], Gamelin [7], Garnett [11], Zalcman [35] show that $\gamma(\cdot)$ is applicable to study approximation problems, and Ahlfors-Beurling [2], Pommerenke [22], Suita [30], [31], [32] study $\gamma(\cdot)$ from the point of view of a conformal invariant. The author studied $\gamma(\cdot)$ in terms of integral geometry [17], [18] and fluid dynamics [19].

In this paper, we are concerned with the variation of $\gamma(E)$ for a small change of $E$. The approach in this direction is given by Havinson [13], Garabedian-Schiffer [10], Schiffer-Hawley [27], Schiffer-Spencer [28], Smith [29]. We shall discuss, in this paper, a variation of $\gamma(E)$ for a

\footnotetext{
Received July 14, 1990.
} 
finite union $E$ of arcs. Here are two motivations to deal with such a set. Given a compact set $F \subset \mathbf{C}$ with a smooth boundary, we can study the Hadamard variation and the Schiffer variation [25]. Then we obtain a finite union $E$ of $\operatorname{arcs}$ such that $\gamma(F)$ is expressed as a perturbation from $\gamma(E)$ and the number of components of $E$ is less than or equal to that of $F$. Thus, in order to get global properties, it is necessary to study a variation of $r(E)$. Another motivation is as follows. Given a compact set $F$, we can find a finite union $G$ of closed disks so that $|\gamma(F)-\gamma(G)|$ is arbitrarily small. Removing some arcs from $G$, we obtain a finite union $E$ of arcs such that $|\gamma(G)-\gamma(F)|$ is arbitrarily small and the number of components of $E$ is less than or equal to that of $F$. Thus a variation of $\gamma(E)$ is necessary. As is seen in the second motivation, for our variation, we may restrict our attention to compact sets $E$ such that $E$ consists of a finite number of mutually disjoint arcs $\left\{C_{j}\right\}_{j=1}^{n}$ and each $\operatorname{arc} C_{j}$ is expressed as a finite union of closed analytic arcs, where a closed arc is analytic if it is contained in an open analytic arc. Let $\mathscr{A}$ denote the totality of such compact sets and let $\mathscr{F}$ denote the totality of domains $\Omega$ such that $\Omega^{c} \in \mathscr{A}$. For $E \in \mathscr{A}$, it is natural to study $\gamma(E)$ in terms of the arc-length $|d z|$. From this point of view, we shall focus on the variation of $\gamma(E)$ with respect to $|d z|$, and, as application of our results, we shall study the structure of $\gamma(A \cup B)$ for two bounded continua $A$ and $B$. For $f \in H^{\infty}\left(\Omega_{E}\right)$, $-f^{\prime}(\infty)$ is the $1 / z$-coefficient of $f$ at $\infty$ and, in the case where $E$ is connected, the Ahlfors function (i.e., the function attaining $\gamma(E)$ ) is nothing but Riemann's mapping from $\Omega_{E}$ onto the unit disk $\mathbf{D}$. Thus our principle is related to Löwner's differential equation for multiply-connected domains (cf. [15, p. 116]). In fact, we shall deduce, in $\S 9$, Löwner's D. E. for simplyconnected domains [33, p. 387] from our formula in $\S 3$. For $\Omega \in \mathscr{F}, \partial \Omega$ denotes the boundary of $\Omega$ having two sides; to each $z \in F$ ( $F=\Omega^{\mathrm{c}}-$ \{endpoints\}), there correspond two points $z_{ \pm} \in \partial \Omega$. Let $H^{2}(\Omega)$ denote the $H^{2}$-space of analytic functions in $\Omega$ such that

$$
\begin{aligned}
& \text { (1) } \lim _{\delta \downarrow 0} \int_{S(\zeta, \delta)}|f(z)|^{2}|d z|=0(\zeta \in\{\text { endpoints of } F\}), \\
& \text { (2) }\|f\|_{H^{2}}=\left(\int_{F}\left\{\left|f\left(z_{+}\right)\right|^{2}+\left|f\left(z_{-}\right)\right|^{2}\right\}|d z|\right)^{1 / 2}<\infty
\end{aligned}
$$

where $f\left(z_{\varepsilon}\right)$ is the non-tangential limit of $f$ at $z_{\varepsilon} \in \partial \Omega(\varepsilon= \pm)$ and $S(\zeta, \delta)$ is the circle of center $\zeta$ and radius $\delta$. For $\Omega \in \mathscr{F}$, there uniquely exists a pair $(g(\cdot ; \Omega), \phi(\cdot ; \Omega))$ of functions in $H^{2}(\Omega)$ such that $g(\infty ; \Omega)=0$, 
$\phi(\infty ; \Omega)=1$ and

( 3 ) $\frac{1}{i} \phi(z ; \Omega) d z=\overline{g(z ; \Omega)}|d z|$ almost everywhere (a.e.) on $\partial \Omega$,

where the orientation of $d z$ is chosen so that $\Omega$ lies to the left [8]. For any $\zeta \in \Omega-\{\infty\}$, there exists uniquely a pair $(K(\cdot, \zeta ; \Omega), L(\cdot, \zeta ; \Omega))$ of functions such that $K(\cdot, \bar{\zeta} ; \Omega),(\cdot-\zeta) L(\cdot, \zeta ; \Omega) \in H^{2}(\Omega), K(\infty, \bar{\zeta} ; \Omega)=L(\infty, \zeta ; \Omega)=0$, $\lim _{z \rightarrow \zeta}(z-\zeta) L(z, \zeta ; \Omega)=1$ and

(4) $\frac{1}{i} L(z, \zeta ; \Omega) d z=\overline{K(z, \bar{\zeta} ; \Omega)}|d z|$ a.e. on $\partial \Omega[4]$.

The kernel $K(z, \bar{\zeta} ; \Omega)$ is called the Szegö kernel and $L(z, \zeta ; \Omega)$ is called the $L$-kernel [4]. (The Szegö kernel is written by $\hat{K}$ in [4]. In this note, we use the notation $K$.) For $z, \zeta \in \Omega-\{\infty\}, z \neq \zeta$, we define

$$
\begin{aligned}
& D c(z, \infty ; \Omega)=|\phi(z ; \Omega)|^{2}-|g(z ; \Omega)|^{2}, \\
& D^{2} c(z, \zeta, \infty ; \Omega)=2 \operatorname{Re}[\{L(z, \zeta ; \Omega) \overline{g(z ; \Omega)}-\overline{K(z, \bar{\zeta} ; \Omega)} \phi(z ; \Omega)\} \overline{\phi(\zeta ; \Omega)} \\
& -\{L(z, \zeta ; \Omega) \overline{\phi(z ; \Omega)}-\overline{K(z, \bar{\zeta} ; \Omega)} g(z ; \Omega)\} \overline{g(\zeta ; \Omega)}] \text {, }
\end{aligned}
$$

where Re denotes the real part. For a closed analytic arc $\Gamma$, a continuous function $w_{t} \in \Gamma$ on an interval $[0,|\Gamma|](|\Gamma|$ is the length of $\Gamma)$ is called the arc-length representation of $\Gamma$, if $w_{0}, w_{|\Gamma|}$ are endpoints of $\Gamma$ and $\left|\Gamma_{t}\right|=t$ $(0 \leq t \leq|\Gamma|)$, where $\Gamma_{t}=\left\{w_{s} ; 0 \leq s \leq t\right\}$. For $\Omega \in \mathscr{F}$ such that $\Omega-\Gamma \in \mathscr{F}$ and $\Omega^{c} \cap \Gamma$ is at most a singleton, we write $\Omega_{t}=\Omega-\Gamma_{t}(0 \leq t \leq|\Gamma|)$; we assume that $\Omega^{c} \cap \Gamma=\left\{w_{0}\right\}$ if $\Omega^{c} \cap \Gamma \neq \emptyset$. We shall show

\section{THEOREM 1.}

(5) For any $0<t \leq|\Gamma|$, the derivative $\partial c\left(\infty ; \Omega_{t}\right) / \partial t$, the limit $\lim _{u \downarrow t} D c\left(w_{u}, \infty ; \Omega_{t}\right)\left(=D c\left(w_{t}, \infty ; \Omega_{t}\right)\right.$, say $)$ exist and $\partial c\left(\infty ; \Omega_{t}\right) / \partial t$ $=D c\left(w_{t}, \infty ; \Omega_{t}\right) / 4$. The right-derivative $\partial c\left(\infty ; \Omega_{0}\right) / \partial t$ at $t=0$ exists and $\partial c\left(\infty ; \Omega_{t}\right) / \partial t$ is continuous on $[0,|\Gamma|]$.

(6) For any $0<t \leq|\Gamma|$ and any $z \in \Omega-(\Gamma \cup\{\infty\})$, the derivative $\partial D c\left(z, \infty ; \Omega_{t}\right) / \partial t$, the limit $\lim _{u \downarrow t} D^{2} c\left(w_{u}, z, \infty ; \Omega_{t}\right)\left(=D^{2} c\left(w_{t}, z, \infty ; \Omega_{t}\right)\right.$, say) exist and $\partial D c\left(z, \infty ; \Omega_{t}\right) / \partial t=D^{2} c\left(w_{t}, z, \infty ; \Omega_{t}\right) / 4$. For any $z \in \Omega$ $-(\Gamma \cup\{\infty\})$, the right-derivative $\partial D\left(z, \infty ; \Omega_{0}\right) / \partial t$ at $t=0$ exists and $\partial D c\left(z, \infty ; \Omega_{t}\right) / \partial t$ is continuous on $[0,|\Gamma|]$.

In this theorem, the derivative and the limit at $t=|\Gamma|$ are defined by the arc-length representation of an open analytic arc containing $\Gamma$. The right-derivative $\partial c\left(\infty ; \Omega_{0}\right) / \partial t$ is not, in general, equal to $D c\left(w_{0}, \infty ; \Omega_{0}\right) / 4$. 
(See Remark 19.) To study $D c(z, \infty ; \Omega)$ and $D^{2} c(z, \zeta, \infty ; \Omega)$, we introduce a wider class of domains. Let $\mathscr{G}$ denote the totality of domains $\Omega$ with the following property: $\Omega$ is expressed as $\Omega=\Omega^{*}-E$ with $E \in \mathscr{A}$ and a domain $\Omega^{*}, \Omega^{*} \supset E$ bounded by a finite number of Jordan curves $\left\{C_{j}\right\}_{j=1}^{n}$ such that each $C_{j}$ is a finite union of closed analytic arcs. For $\Omega \in \mathscr{G}$, the Szegö kernel and the $L$-kernel exist; the condition at $\infty$ is removed if $\Omega \nexists \infty$. For three distinct numbers $w, z, \zeta \in \Omega$, we define

$$
\begin{aligned}
& D c(z, \zeta ; \Omega)=|L(z, \zeta ; \Omega)|^{2}-|K(z, \zeta ; \Omega)|^{2}, \\
& D^{2} c(w, z, \zeta ; \Omega)= 2 \operatorname{Re}\{D L(w, z, \zeta ; \Omega) \overline{L(z, \zeta ; \Omega)} \\
& \quad-D K(w, z, \zeta ; \Omega) \overline{K(z, \zeta ; \Omega)\}}
\end{aligned}
$$

where

$$
\begin{aligned}
& D K(w, z, \zeta ; \Omega)=L(w, z ; \Omega) \overline{L(w, \zeta ; \Omega)}-\overline{K(w, \bar{z} ; \Omega)} K(w, \bar{\zeta} ; \Omega), \\
& D L(w, z, \zeta ; \Omega)=L(w, z ; \Omega) \overline{K(w, \zeta ; \Omega)}-\overline{K(w, \bar{z} ; \Omega)} L(w, \zeta ; \Omega) .
\end{aligned}
$$

In the above definition, we replace $K(\cdot, \bar{\infty} ; \Omega)=\overline{K(\infty, \cdot ; \Omega)}$ by $-g(\cdot ; \Omega)$, and replace $L(\cdot, \infty ; \Omega)=-L(\infty, \cdot ; \Omega)$ by $-\phi(\cdot ; \Omega)$ if $\Omega \ni \infty$ and one of $w, z, \zeta$ is $\infty$. The following proposition plays an important role in our conformal geometry.

Proposition 2. The differential forms $D c(z, \zeta ; \Omega)|d z||d \zeta|$ and $D^{2} c(w, z, \zeta ; \Omega)|d w\|d z\| d \zeta|$ are conformally invariant, $D c(z, \zeta ; \Omega)=D c(\zeta, z ; \Omega)$ and $D^{2} c(w, z, \zeta ; \Omega)$ is invariant for any permutation of a triple $(w, z, \zeta)$.

In this proposition, $D c(z, \zeta ; \Omega)|d z||d \zeta|$ is conformally invariant in the following sense: $D c(z, \zeta ; \Omega)=D c(h(z), h(\zeta) ; h(\Omega))\left|h^{\prime}(z) h^{\prime}(\zeta)\right|$, if $h$ is conformal, where

$$
h^{\prime}(z)=\left\{\begin{array}{l}
\lim _{\zeta \rightarrow \infty} \zeta\{h(\zeta)-h(\infty)\} \quad \text { if } z=\infty, h(\infty) \neq \infty \\
\lim _{\zeta \rightarrow \infty} h(\zeta) / \zeta \quad \text { if } z=\infty, h(\infty)=\infty \\
\lim _{\zeta \rightarrow z}(z-\zeta) h(\zeta) \text { if } z \neq \infty, h(z)=\infty .
\end{array}\right.
$$

The meaning of the conformal invariance of $D^{2} c(w, z, \zeta ; \Omega)|d w\|d z\| d \zeta|$ is analogous. Our results are applied as follows. Given $E \in \mathscr{A}$, we can write $E=C_{1} \cup \cdots \cup C_{n}$ with mutually disjoint ares $\left\{C_{j}\right\}_{j=1}^{n}$. Using the arc-length representations of $C_{j}(1 \leq j \leq n)$, we define a right-continuous arc-length representation $W_{t}(0 \leq t \leq|E|)$ of $E$. Let $\Omega_{t}=\left\{W_{s} ; 0 \leq s \leq t\right\}^{c}$ $(0 \leq t \leq|E|)$. Then $c\left(\infty ; \Omega_{t}\right)$ is continuous on $[0,|E|]$. Theorem 1 shows that 


$$
\text { ( 7) } \quad \gamma(E)=c\left(\infty ; E^{c}\right)=\frac{1}{4} \int_{0}^{|E|} D c\left(W_{\iota}, \infty ; \Omega_{t}\right) d t .
$$

The quantity $D c\left(W_{\iota}, \infty ; \Omega_{t}\right)$ is defined by the limit $\lim _{u \downarrow t} D c\left(W_{u}, \infty ; \Omega_{t}\right)$. Thus, to study $D c\left(W_{t}, \infty ; \Omega_{t}\right)$, it is sufficient to investigate $D c\left(W_{u}, \infty ; \Omega_{t}\right)$ for $u>t$ sufficiently near to $t$. Given $0<t<|E|$ and $z \in \Omega_{t}-\{\infty\}$, we take a right-continuous arc-length representation $W_{s}^{*}(0 \leq s \leq t)$ of $\Omega_{t}^{c} ; W_{s}^{*}$ may not be equal to $W_{s}$, in general. Since $D c\left(z, \infty ; \Omega_{s}^{*}\right)\left(\Omega_{s}^{*}=\left\{W_{u}^{*}\right.\right.$; $\left.0 \leq u \leq s\}^{c}\right)$ is continuous on $[0, t]$, we have

$$
\begin{array}{r}
D c\left(z, \infty ; \Omega_{t}\right)=D c\left(z, \infty ; \Omega_{s}^{*}\right)+\frac{1}{4} \int_{s}^{t} D^{2} c\left(W_{u}^{*}, z, \infty ; \Omega_{u}^{*}\right) d u \\
(0 \leq s \leq t) .
\end{array}
$$

Using Proposition 2, we can study $D^{2} c(w, z, \infty ; \Omega)$ by taking conformal mappings from $\Omega$ onto canonical domains. We shall show

THeOREM 3. If $\Omega$ is simply or doubly connected, then $D^{2} c(w, z, \zeta ; \Omega) \leq 0$.

As application of this theorem, we here deduce Suita's subadditivity [32]: $\gamma(A \cup B) \leq \gamma(A)+\gamma(B)$, if $A$ and $B$ are disjoint two continua. For the sake of simplicity, we work only with $A, B \in \mathscr{A}$. Using (7) with $E=$ $A \cup B$ and a right-continuous arc-length representation $W_{t}(0 \leq t \leq|A \cup B|)$ such that $W_{0} \in A$, we have

$$
r(A \cup B)=r(A)+\frac{1}{4} \int_{|A|}^{|A \cup B|} D c\left(W_{\iota}, \infty ; \Omega_{\iota}\right) d t .
$$

For $|A|<t \leq|A \cup B|$, we take an arc-length representation $W_{s}^{*}(0 \leq s \leq t)$ of $\left\{W_{s} ; 0 \leq s \leq t\right\}$ so that $W_{0}^{*}=W_{|A|}(\in B)$. Then $W_{t-|A|}^{*}=W_{\iota}$. Using (8), we have, with $\Omega_{s}^{*}=\left\{W_{u}^{*} ; 0 \leq u \leq s\right\}^{c}$,

$$
\begin{aligned}
D c\left(z, \infty ; \Omega_{t}\right) & =D c\left(z, \infty ; \Omega_{t-|A|}^{*}\right)+\frac{1}{4} \int_{t-|A|}^{t} D^{2} c\left(W_{s}^{*}, z, \infty ; \Omega_{s}^{*}\right) d s \\
& \leq D c\left(z, \infty ; \Omega_{t-|A|}^{*}\right) \quad\left(z \in \Omega_{t}-\{\infty\}\right) .
\end{aligned}
$$

Letting $z$ tend to $W_{t-|A|}^{*}=W_{t}$ along $B$, we obtain $D c\left(W_{t}, \infty ; \Omega_{t}\right) \leq$ $D c\left(W_{t-|A|}^{*}, \infty ; \Omega_{t-|A|}^{*}\right)$. Since

$$
\frac{1}{4} \int_{|A|}^{|A \cup B|} D c\left(W_{t}, \infty ; \Omega_{t}\right) d t \leq \frac{1}{4} \int_{|A|}^{|A \cup B|} D c\left(W_{t-|A|}^{*}, \infty ; \Omega_{t-|A|}^{*}\right) d t=\gamma(B),
$$


we obtain $\gamma(A \cup B) \leq \gamma(A)+\gamma(B)$. Suita's method is based on Rengel's inequality [33, p. 393] which is an application of Bieberbach's area method. On the other hand, our method is based on a differential equation for arcs and yields a quantitative estimate of $\gamma(A)+\gamma(B)-\gamma(A \cup B)$. The inequality $D^{2} c \leq 0$ for simply-connected domains is closely related to the triangle inequality with respect to the hyperbolic distance. The study of $D^{2} c$ for doubly-connected domains is related to the theory of elliptic functions [12] and the proof of $D^{2} c \leq 0$ consequently reduces to an inequality of Möbius type in elementary geometry:

$$
\begin{aligned}
\frac{Q Q^{\prime} r^{\prime}}{R R^{\prime} q q^{\prime}}+\frac{R R^{\prime} q q^{\prime}}{Q Q^{\prime} r r^{\prime}} \leq & 2 \cos \left(\alpha+\alpha^{\prime}\right) \cos \left(\beta+\beta^{\prime}\right) \\
& +\frac{s^{2}+t^{2}}{s t} \sin \left(\alpha+\alpha^{\prime}\right) \sin \left(\beta+\beta^{\prime}\right) .
\end{aligned}
$$

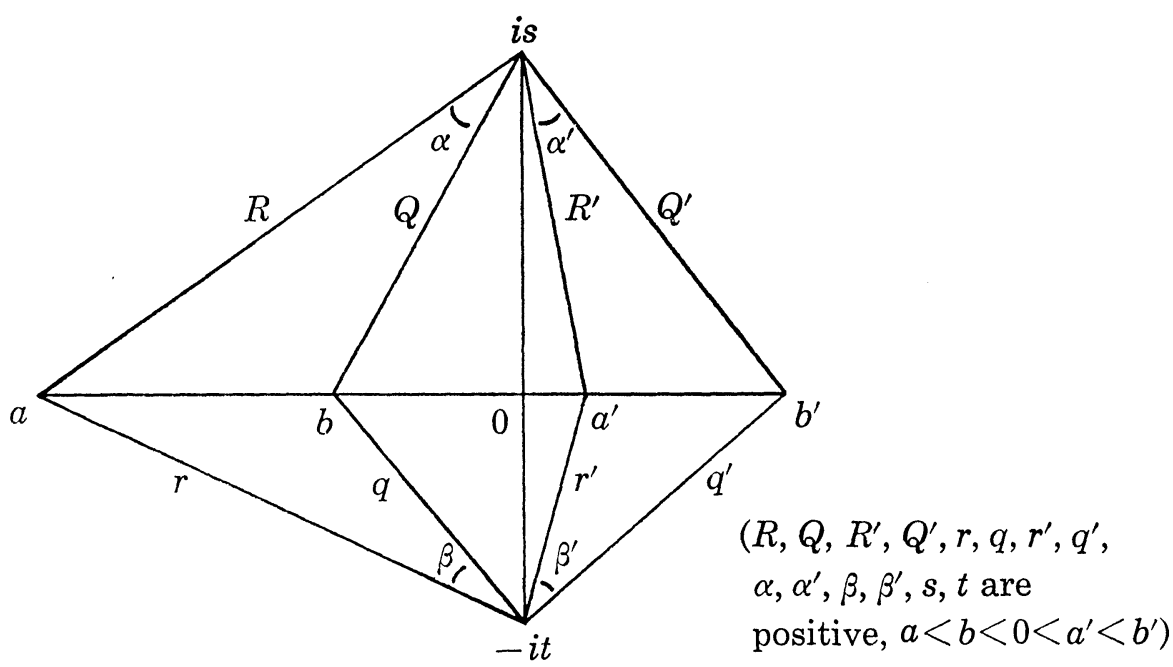

In $\S \S 8-9$, we shall study the structure of $D^{2} c$ for simply and doubly connected domains. For general multiply-connected domains, the behaviour of $D^{2} c$ is very complicated, and we do not refer to the general case in this paper. In $\S 2$, we shall prepare some elementary properties which will be used later. In $\S 3$, we shall show a formula for Szegö kernels and shall deduce Theorem 1 from this formula. The proof of the formula will be given in $\S \S 4-7$. The proof of Theorem 3 will be given in $\S \S 8-9$. 


\section{§2. Elementary properties}

In this section, we show some elementary properties which are used later. For $p \geq 1$ and $\Omega \in \mathscr{G}, H^{p}(\Omega)$ denotes the $H^{p}$-space of functions in $\Omega$ defined by (1) and (2) with the power 2 replaced by $p ; \partial \Omega$ has two sides on the components of $\Omega^{c}$ corresponding to arcs, $\partial \Omega$ is single on the components of $\Omega^{c}$ corresponding to Jordan curves, and the integral is taken over all $\partial \Omega$ with respect to $|d z|$. We put

$$
\begin{gathered}
c^{*}(\zeta ; \Omega)=\inf \left\{\frac{1}{2 \pi} \int_{\partial \Omega}\left|\frac{\psi(w)}{w-\zeta}\right|^{2}|d w| ; \psi \in H^{2}(\Omega), \psi(\zeta)=1\right\} \quad(\zeta \neq \infty), \\
c^{*}(\infty ; \Omega)=\inf \left\{\frac{1}{2 \pi}\|\phi\|_{H^{2}}^{2} ; \phi \in H^{2}(\Omega), \phi(\infty)=1\right\} \quad(\Omega \ni \infty) .
\end{gathered}
$$

Then $c^{*}(\zeta ; \Omega)=c(\zeta ; \Omega)(\zeta \in \Omega)$ [8]. The pair $(K(z, \bar{\zeta} ; \Omega), L(z, \zeta ; \Omega))$ of the Szegö kernel and the $L$-kernel is unique and satisfies

$$
K(z, \bar{\zeta} ; \Omega)=\overline{K(\zeta, \bar{z} ; \Omega)}, \quad L(z, \zeta ; \Omega)=-L(\zeta, z ; \Omega) .
$$

The Szegö kernel $K(z, \bar{\zeta} ; \Omega)$ is a reproducing kernel in the following sense:

$$
f(\zeta)=\frac{1}{2 \pi} \int_{\partial \Omega} \overline{K(z, \bar{\zeta} ; \Omega)} f(z)|d z| \quad\left(f \in H^{2}(\Omega), f(\infty)=0\right)[4]
$$

Making a double of $\Omega$, we can treat the pair as a kernel on the Riemann surface. When no confusion can arise, we write simply $K(z, \bar{\zeta})$ and $L(z, \zeta)$. Let $D(z, r)$ denote the open disk of center $z$ and radius $r$, and let $\mathbf{D}=$ $D(0,1)$. We remark

Lemma 4. Let $\Omega \ni \infty$. Then $\phi(z ; \Omega)$ is the function attaining $c^{*}(\infty ; \Omega)$ and

$$
g(z ; \Omega)=\frac{1}{2 \pi} \int_{\partial \Omega} \frac{1}{w-z} \overline{\phi(} \overline{w ; \Omega)}|d w|
$$

Let $\zeta \neq \infty$. Then

(9) $K(z, \bar{\zeta})=\frac{1}{2 \pi} \int_{\partial \Omega} \frac{1}{(w-z) \overline{(w-\zeta)}} \overline{\psi_{\zeta}(w)}|d w|$,

$$
L(z, \zeta)=\psi_{\zeta}(z) /(z-\zeta),
$$

where $\psi_{\zeta}$ is the function attaining $c^{*}(\zeta ; \Omega)$.

This lemma is essentially known in [4], [8]. Only (9) is not evident 
in the case of $\Omega \ni \infty$. Let $\zeta \in \Omega-\{\infty\}$. Since $\psi_{\zeta}$ is the solution of the minimum problem $c^{*}(\zeta ; \Omega)$, a measure $|w-\zeta|^{-2} \overline{\psi_{\zeta}(w)}|d w|$ on $\partial \Omega$ annihilates all $g \in H^{2}(\Omega), g(\zeta)=0$. Thus

$$
\frac{1}{|w-\zeta|^{2}} \overline{\psi_{\zeta}(w)}|d w|-\frac{c}{2 \pi i(w-\zeta)} d w \quad\left(c=\int_{\partial \Omega} \frac{1}{|w-\zeta|^{2}} \overline{\psi_{\zeta}(w)}|d w|\right)
$$

annihilates all $g \in H^{2}(\Omega), g(\infty)=0$. By Riesz's theorem, we can write

$$
\frac{1}{|w-\zeta|^{2}} \overline{\psi_{\zeta}(w)}|d w|=\left\{\psi_{\zeta}^{*}(w)+\frac{c}{2 \pi i(w-\zeta)}\right\} d w
$$

on $\partial \Omega$ with some $\psi_{\zeta}^{*} \in H^{1}(\Omega), \psi_{\zeta}^{*}(\infty)=0\left[11\right.$, p. 15]. Note that $c=\int_{\partial \Omega} \psi_{\zeta}^{*}(w) d w$. Let $K^{*}(z, \bar{\zeta})$ denote the kernel in the right-hand side of (9). Then, for almost all $z$ on $\partial \Omega$,

$$
\begin{aligned}
K^{*}(z, \bar{\zeta}) & =\frac{1}{2 \pi} \int_{\partial \Omega} \frac{w-\zeta}{w-z} \frac{1}{|w-\zeta|^{2}} \overline{\psi_{\zeta}(w) \mid} d w \mid \\
& =\frac{1}{2 \pi} \int_{\partial \Omega} \frac{w-\zeta}{w-z}\left\{\psi_{\zeta}^{*}(w)+\frac{c}{2 \pi i(w-\zeta)}\right\} d w \\
& =\frac{1}{2 \pi} \int_{\partial \Omega}\left\{\frac{z-\zeta}{w-z}+1\right\} \psi_{\zeta}^{*}(w) d w \\
& =i(z-\zeta)\left\{\psi_{\zeta}^{*}(z)+\frac{c}{2 \pi i(z-\zeta)}\right\},
\end{aligned}
$$

which shows that

$$
\begin{aligned}
& K^{*}(z, \bar{\zeta}) d z=i \overline{\psi_{\zeta}(z) /(z-\zeta)}|d z| \text { a.e. on } \partial \Omega \text {, i.e., } \\
& \frac{1}{i} \psi_{\zeta}(z) /(z-\zeta) d z=\overline{K^{*}(z, \bar{\zeta})}|d z| \text { a.e. on } \partial \Omega \text {. }
\end{aligned}
$$

Thus the pair $\left(K^{*}(z, \bar{\zeta}), \psi_{\xi}(z) /(z-\zeta)\right)$ satisfies (4). From the form of the integrand in (9), we see that $K^{*}(z, \bar{\zeta})$ has no inner function, and hence this pair satisfies all the conditions of the Szegö kernel and the $L$-kernel. Since $(K, L)$ is unique, we have $K(z, \bar{\zeta})=K^{*}(z, \bar{\zeta})$ and $L(z, \zeta)=\psi_{\zeta}(z) /(z-\zeta)$.

Lemma 5. Let $\Omega \ni \infty$ and $z \in \Omega-\{\infty\}$. Then

$$
\begin{aligned}
& g(z ; \Omega)=\frac{1}{2 \pi} \int_{\partial \Omega} L(\zeta, z)|d \zeta|=\frac{1}{2 \pi i} \int_{\partial \Omega} K(\zeta, \bar{z}) d \zeta \\
& \phi(z ; \Omega)=1-\frac{1}{2 \pi} \int_{\partial \Omega} \overline{K(\zeta, \bar{z})}|d \zeta|=1-\frac{1}{2 \pi i} \int_{\partial \Omega} L(\zeta, z) d \zeta
\end{aligned}
$$

In fact, (4) shows that 


$$
\begin{aligned}
& g(z ; \Omega)=\frac{1}{2 \pi i} \int_{\partial \Omega} \frac{1}{\zeta-z} g(\zeta ; \Omega) d \zeta=\frac{1}{2 \pi i} \int_{\partial \Omega} L(\zeta, z) g(\zeta ; \Omega) d \zeta \\
& =\frac{1}{2 \pi} \int_{\partial \Omega} \overline{K(\zeta, \bar{z})} g(\zeta ; \Omega)|d \zeta|=\overline{\frac{1}{2 \pi i} \int_{\partial \Omega} K(\zeta, \bar{z}) \phi(\zeta ; \Omega) d \zeta} \\
& =\overline{\frac{1}{2 \pi i} \int_{\partial \Omega} K(\zeta, \bar{z}) d \zeta}=\frac{1}{2 \pi} \int_{\partial \Omega} L(\zeta, z)|d \zeta| .
\end{aligned}
$$

Since

$$
\begin{aligned}
\frac{1}{2 \pi i} \int_{\partial \Omega} L(\zeta, z) \phi(\zeta ; \Omega) d \zeta & =\frac{1}{2 \pi} \int_{\partial \Omega} \overline{K(\zeta, \bar{z})} \phi(\zeta ; \Omega)|d \zeta| \\
& =\frac{-\overline{1}}{2 \pi i} \int_{\partial \Omega} K(\zeta, \bar{z}) g \overline{(\zeta ; \Omega) d \zeta}=0,
\end{aligned}
$$

we have

$$
\begin{aligned}
\phi(z ; \Omega) & =1+\frac{1}{2 \pi i} \int_{\partial \Omega} \frac{1}{\zeta-z}\{\phi(\zeta ; \Omega)-1\} d \zeta \\
& =1+\frac{1}{2 \pi i} \int_{\partial \Omega} L(\zeta, z)\{\phi(\zeta ; \Omega)-1\} d \zeta \\
& =1-\frac{1}{2 \pi i} \int_{\partial \Omega} L(\zeta, z) d \zeta=1-\frac{1}{2 \pi} \int_{\partial \Omega} \overline{K(\zeta, \bar{z})}|d \zeta|
\end{aligned}
$$

This lemma plays an important role to study $\phi(z ; \Omega) ; \phi(z ; \Omega)^{2}$ is called the Garabedian function of $\Omega^{c}[11$, p. 19]. The Ahlfors function $f(z ; \Omega)$ of $\Omega^{c}$ (i.e., $f(\cdot ; \Omega) \in H^{\infty}(\Omega),\|f(\cdot ; \Omega)\|_{H^{\infty}}=1, f^{\prime}(\infty ; \Omega)=\gamma\left(\Omega^{c}\right)$ ) is expressed as $g(z ; \Omega) / \phi(z ; \Omega)[11$, pp. 18-24]. Thus (10) gives that

$$
\phi(z ; \Omega)=f(z ; \Omega)^{-1} \overline{\frac{1}{2 \pi i} \int_{\partial D(0, r) c} K(\zeta, \bar{z}) d \zeta}
$$

for $r>0$ satisfying $D(0, r)^{c} \subset \Omega$. Using this expression, we can define the Garabedian function of any domain (cf. [29], [30]).

Lemma 6. Let $\Omega \ni 0, \infty$ and $z \in \Omega-\{0, \infty\}$. Then

(12) $g(z ; \Omega)=-K(\tilde{z}, \overline{0} ; \tilde{\Omega}) \tilde{z}, \quad \phi(z ; \Omega)=L(\tilde{z}, 0 ; \tilde{\Omega}) \tilde{z}$,

(13) $g(0 ; \tilde{\Omega})=\overline{g(0 ; \Omega)}, \quad \phi(0 ; \tilde{\Omega})=\phi(0 ; \Omega)$,

where $\tilde{\Omega}=\{\tilde{\zeta}=1 / \zeta ; \zeta \in \Omega\}$.

To see (12), we put

$$
\tilde{g}(\tilde{z})=-g(1 / \tilde{z} ; \Omega) / \tilde{z}, \quad \tilde{\phi}(\tilde{z})=\phi(1 / \tilde{z} ; \Omega) / \tilde{z}(\tilde{z} \in \tilde{\Omega}) .
$$


Then $\tilde{g}, \tilde{\phi} \tilde{z} \in H^{2}(\tilde{\Omega}), \tilde{g}(\infty)=0, \lim _{\tilde{z} \rightarrow 0} \tilde{\phi}(\tilde{z}) \tilde{z}=1$ and

$$
\begin{aligned}
\frac{1}{i} \tilde{\phi}(\tilde{z}) d \tilde{z} & =-\frac{1}{i} \phi(z ; \Omega) z d z / z^{2} \\
& =-\overline{g(z ; \Omega)}|d z||z=\overline{\tilde{g}(\tilde{z})}| d \tilde{z} \mid \text { a.e. on } \partial \Omega .
\end{aligned}
$$

Thus the unicity yields that $\tilde{g}(\tilde{z})=K(\tilde{z}, \overline{0} ; \tilde{\Omega})$ and $\tilde{\phi}(\tilde{z})=L(\tilde{z}, 0 ; \tilde{\Omega})$, i.e., (12). Combined with (10) and (11), it follows that

$$
\begin{aligned}
& g(0 ; \tilde{\Omega})=-\lim _{\tilde{\zeta} \rightarrow \infty} \overline{K(\tilde{\zeta}, \overline{0} ; \tilde{\Omega}) \tilde{\zeta}}=\overline{g(0 ; \Omega)}, \\
& \phi(0 ; \tilde{\Omega})=\lim _{\tilde{\zeta} \rightarrow \infty} L(\tilde{\zeta}, 0 ; \tilde{\Omega}) \tilde{\zeta}=\phi(0 ; \Omega) .
\end{aligned}
$$

Thus (13) holds.

As application of (13), we have $D c(0, \infty ; \tilde{\Omega})=D c(0, \infty ; \Omega) . \quad$ If $\Omega=\tilde{\Omega}$, then $g(0 ; \Omega)$ is real.

Lemma 7. Let $\Omega \ni 0, \infty$. Then

$$
\begin{aligned}
\gamma\left(\Omega^{c}\right) & =c(\infty ; \Omega)=K(0, \overline{0} ; \tilde{\Omega}) \\
& =\lim _{r \rightarrow \infty} \frac{1}{(2 \pi)^{2}} \int_{\partial D(0, r) c} \int_{\partial D(0, r) c} K(z, \bar{\zeta} ; \Omega) d z \overline{d \zeta} .
\end{aligned}
$$

In fact, (10) shows that, for $r>0$ satisfying $D(0, r)^{c} \subset \Omega$,

$$
\begin{aligned}
c(\infty ; \Omega) & =c^{*}(\infty ; \Omega)=\frac{1}{2 \pi} \int_{\partial \Omega}|\phi(w ; \Omega)|^{2}|d w| \\
& =\frac{1}{2 \pi} \int_{\partial \Omega}|g(w ; \Omega)|^{2}|d w|=\frac{1}{2 \pi} \int_{\partial \Omega}\left|\frac{1}{2 \pi} \int_{\partial D(0, r)^{c}} K(z, \bar{w}) d z\right|^{2}|d w| \\
& =\frac{1}{(2 \pi)^{2}} \int_{\partial D(0, r)^{c}} \int_{\partial D(0, r) c}\left\{\frac{1}{2 \pi} \int_{\partial \Omega} K(z, \bar{w}) \overline{K(\zeta, \bar{w}) \mid} d w \mid\right\} d z \overline{d \zeta} \\
& =\frac{1}{(2 \pi)^{2}} \int_{\partial D(0, r)^{c}} \int_{\partial D(0, r) c} K(z, \bar{\zeta}) d z \overline{d \zeta} .
\end{aligned}
$$

It is known that $g^{\prime}(\infty ; \Omega)=c(\infty ; \Omega)[11, \mathrm{pp} .18-24]$. Thus the first equality in (12) shows that $c(\infty ; \Omega)=K(0, \overline{0} ; \tilde{\Omega})$.

Here is a version of the Hadamard variation to Szegö kernels. For a domain $\Omega \ni \infty$ whose boundary consists of a finite number of analytic Jordan curves, we take a non-negative smooth function $\rho$ on $\partial \Omega$. For a sufficiently small number $\varepsilon>0$, let $\Omega_{\varepsilon}$ denote a domain bounded by $\xi_{\varepsilon}=$ $\xi+i \varepsilon \rho(\xi) d \xi /|d \xi|, \quad \xi \in \partial \Omega$ containing $\infty$. The variations of the Szegö kernel and the $L$-kernel with respect to $\rho$ are defined by 


$$
D_{\rho} J(z, \cdot ; \Omega)=\lim _{\varepsilon \downarrow 0} \frac{1}{\varepsilon}\left\{J\left(z, \cdot ; \Omega_{\varepsilon}\right)-J(z, \cdot ; \Omega)\right\} \quad(J=K, L) .
$$

Then

$$
\text { (14) } D_{\rho} J(z, \cdot ; \Omega)=\frac{1}{4 \pi} \int_{\partial \Omega} \frac{\partial}{\partial n_{\rho}} D J(\xi, z, \cdot ; \Omega) \rho(\xi)|d \xi| \quad(J=K, L),
$$

where $\partial / \partial n_{\rho}$ is the inner normal derivative at $\xi \in \partial \Omega$.

This equality plays an important role in the study of the capacity $K(z, \bar{z} ; \Omega)$. Since

$$
D K(\xi, z, z ; \Omega)=D c(\xi, z ; \Omega)>0 \quad(\xi \in \Omega)
$$

and $=0 \quad(\xi \in \partial \Omega)$, we have $\partial D K(\xi, z, z ; \Omega) / \partial n_{\rho} \geq 0 \quad(\xi \in \partial \Omega)$, and hence $D_{\rho} K(z, \bar{z} ; \Omega) \geq 0$, by (14). Thus $K(z, \bar{z} ; \Omega)$ is monotone with respect to $\Omega$. This is a typical example how to use (14). In the following section, we shall show a version of (14) to the arc-length variation. The outline of the proof of (14) is as follows. Functions $J(z, \cdot)=J(z, \cdot ; \Omega), J_{\varepsilon}(z, \cdot)=$ $J\left(z, \cdot ; \Omega_{\varepsilon}\right)(J=K, L ; \varepsilon>0)$ are analytic in a neighborhood of $\partial \Omega$ with respect to $z$. The reproducing property yields that

$$
\begin{aligned}
K_{\varepsilon}(z, \bar{\zeta}) & =\frac{1}{2 \pi} \int_{\partial \Omega} K_{\varepsilon}(\xi, \bar{\zeta}) \overline{K(\xi, \bar{z})}|d \xi| \\
K(z, \bar{\zeta}) & =\frac{1}{2 \pi} \int_{\partial \Omega_{\varepsilon}} K_{\varepsilon}(\xi, \bar{\zeta}) \overline{K(\xi, \bar{z})}|d \xi|
\end{aligned}
$$

Studying the difference, we obtain

$$
\begin{aligned}
D_{\rho} K(z, \bar{\zeta})= & \frac{1}{2 \pi i} \int_{\partial \Omega} \frac{\partial K}{\partial \xi}(\xi, \bar{\zeta}) \overline{K(\xi, \bar{z})} \rho(\xi) d \xi \\
& -\frac{1}{2 \pi i} \int_{\partial \Omega} K(\xi, \bar{\zeta}) \overline{\frac{\partial K}{\partial \xi}(\xi, \bar{z})} \rho(\xi) \overline{d \xi} \\
& +\frac{1}{2 \pi} \int_{\partial \Omega} K(\xi, \bar{\zeta}) \overline{K(\xi, \bar{z})} \rho(\xi) d \arg (d \xi)
\end{aligned}
$$

Using (4), we have

$$
\begin{aligned}
& K(z, \bar{\zeta})=\frac{1}{2 \pi} \int_{\partial \Omega} \overline{L_{\varepsilon}(\xi, \zeta)} L(\xi, z)|d \xi| \\
& K_{\varepsilon}(z, \bar{\zeta})=\frac{1}{2 \pi} \int_{\partial \Omega_{\varepsilon}} \overline{L_{\varepsilon}(\xi, \zeta)} L(\xi, z)|d \xi|
\end{aligned}
$$

Studying the difference, we obtain 
(16)

$$
\begin{aligned}
& D_{\rho} K(z, \bar{\zeta})=-\frac{1}{2 \pi i} \int_{\partial \Omega} \overline{L(\xi, \zeta)} \frac{\partial L}{\partial \xi}(\xi, z) \rho(\xi) d \xi \\
& +\frac{1}{2 \pi i} \int_{\partial \Omega} \overline{\frac{\partial L}{\partial \xi}(\xi, \zeta)} L(\xi, z) \rho(\xi) \overline{d \xi} \\
& -\frac{1}{2 \pi} \int_{\partial \Omega} \overline{L(\xi, \zeta)} L(\xi, z) \rho(\xi) d \arg (d \xi) \text {. }
\end{aligned}
$$

Note that $\overline{L(\xi, \zeta)} L(\xi, z)=K(\xi, \bar{\zeta}) \overline{K(\xi, \bar{z})}(\xi \in \partial \Omega)$. Thus, adding (15), (16), and dividing by 2 , we obtain $(14)(J=K)$. Using

$$
\begin{aligned}
L_{\varepsilon}(z, \zeta)-L(z, \zeta) & =\frac{1}{2 \pi} \int_{\partial \Omega} L_{\varepsilon}(\xi, \zeta) \overline{K(\xi, \bar{z})}|d \xi| \\
& =\frac{1}{2 \pi} \int_{\partial \Omega_{\varepsilon}} \overline{K_{\varepsilon}(\xi, \bar{\zeta})} L(\xi, z)|d \xi|,
\end{aligned}
$$

we obtain (14) $(J=L)$.

Finally we remark that, for $z \in \Omega-\{\infty\}$,

$$
\frac{1}{i} \frac{\partial L}{\partial \xi}(\xi, z)(d \xi)^{2}=\frac{\overline{\partial K}}{\partial \xi}(\xi, \bar{z}) d \xi|d \xi|-i \overline{K(\xi, \bar{z})}|d \xi| d \arg (d \xi) \text { on } \xi \in \partial \Omega .
$$

In fact, let $\xi=\xi(t)\left(0 \leq t \leq t_{0}\right)$ be an arc-length representation of $\partial \Omega$ in a neighborhood of $\xi(0) \in \partial \Omega$, and let $\xi^{\prime}(t)=e^{i \theta(t)}$. Then

$$
\begin{aligned}
\frac{1}{i} \frac{\partial L}{\partial \xi}(\xi(t), z) \xi^{\prime}(t) & =\frac{1}{i} \frac{\partial L}{\partial t}(\xi(t), z)=\frac{\partial}{\partial t}\left\{\overline{K(\xi(t), \bar{z})} e^{-i \theta(t)}\right\} \\
& =\frac{\overline{\partial K}}{\partial \xi}(\xi(t), \bar{z}) \xi^{\prime}(t) e^{-i \theta(t)}-i \overline{K(\xi(t), \bar{z})} e^{-i \theta(t)} \theta^{\prime}(t) \\
& =\frac{\partial \bar{K}(\xi(t), \bar{z}) \xi^{\prime}(t)^{2}}{\partial \xi}-i \overline{K(\xi(t), \bar{z}) \xi^{\prime}(t)} \frac{\partial}{\partial t} \arg \left(\xi^{\prime}(t)\right) .
\end{aligned}
$$

Multiplying this quantity by $\xi^{\prime}(t)$, we obtain the required equality. As application, we have, for $z, \zeta \in \Omega-\{\infty\}, z \neq \zeta$,

$$
\begin{aligned}
& \frac{1}{i} \frac{\partial L}{\partial \xi}(\xi, \zeta) \overline{L(\xi, z)} d \xi=\frac{1}{i} \frac{\overline{\partial K}(\xi, \bar{\zeta})}{\partial \xi}(\xi, \bar{z}) \overline{d \xi} \\
& -\overline{K(\xi, \bar{\zeta})} K(\xi, \bar{z}) d \arg (d \xi) \text { on } \xi \in \partial \Omega \text {, } \\
& \frac{1}{i} \frac{\partial L}{\partial \xi}(\xi, \zeta) \overline{K(\xi, \bar{z})} d \xi=\frac{1}{i} \frac{\partial K}{\partial \xi}(\xi, \bar{\zeta}) L(\xi, z) \overline{d \xi} \\
& -\overline{K(\xi, \bar{\zeta})} L(\xi, z) d \arg (d \xi) \text { on } \xi \in \partial \Omega \text {. }
\end{aligned}
$$

Using these identities, we can rewrite (14) $(J=K, L)$ according as our purpose. 


\section{$\S 3$. A formula and deduction of Theorem 1}

To prove Theorem 1, it is necessary to study the Szegö kernels and the $L$-kernels. For $\Omega \in \mathscr{G}$ and a closed analytic arc $\Gamma$ such that $\Omega-\Gamma$ $\in \mathscr{G}$ and $\Omega^{c} \cap \Gamma$ is at most a singleton, we take the arc-length representation $w_{t}(0 \leq t \leq|\Gamma|)$ of $\Gamma$; we assume that $\Omega^{c} \cap \Gamma=\left\{w_{0}\right\}$ if $\Omega^{c} \cap \Gamma \neq \emptyset$. We write $\Gamma_{t}=\left\{w_{s} ; 0 \leq s \leq t\right\}, \Omega_{t}=\Omega-\Gamma_{t}(0 \leq t \leq|\Gamma|)$ and $X=\{(z, \zeta)$; $z, \zeta \in \Omega-(\Gamma \cup\{\infty\})\}$. Recall the definition of $D J(J=K, L)$. We see that $D J\left(w_{u}, z, \zeta ; \Omega_{t}\right) \quad(J=K, L)$ are defined for $0<t<u \leq|\Gamma|,(z, \zeta) \in X$. We shall show

Theorem 8. For any $0<t \leq|\Gamma|$ and any $(z, \zeta) \in X$, the derivative $\partial K\left(z, \bar{\zeta} ; \Omega_{t}\right) / \partial t$, the limit $\lim _{u ! t} D K\left(w_{u}, z, \zeta ; \Omega_{t}\right)\left(=D K\left(w_{t}, z, \zeta ; \Omega_{t}\right)\right.$, say) exist and

$$
\frac{\partial K}{\partial t}\left(z, \bar{\zeta} ; \Omega_{t}\right)=\frac{1}{4} D K\left(w_{t}, z, \zeta ; \Omega_{t}\right)
$$

For any $(z, \zeta) \in X$, the right-derivative $\partial K\left(z, \bar{\zeta} ; \Omega_{0}\right) / \partial t$ at $t=0$ exists and $\partial K\left(z, \bar{\zeta} ; \Omega_{t}\right) / \partial t$ is continuous on $[0,|\Gamma|]$. These properties hold with $K$ replaced by $L$.

In this theorem, we put $\partial L\left(z, z ; \Omega_{t}\right) / \partial t=0$. From the definition of $D L$, we have $D L\left(w_{t}, z, z ; \Omega_{t}\right)=0$, and hence, in the case of $z=\zeta$, (17) holds with $K$ replaced by $L$. Recall that $w_{t}$ is an endpoint of $\Gamma_{t}(0<t \leq|\Gamma|)$. Thus this theorem is near to Löwner's D.E. than Schiffer's variational formula. In $\S 8$, we shall deduce Löwner's D. E. for simply-connected domains from this theorem.

Once this theorem is established, Theorem 1 is deduced as follows. Note that, for $R \in \mathscr{G}, R \ni 0, \infty$ and $w, z \in R-\{0, \infty\}, w \neq z$,

(18) $D c(w, \infty ; R)=D K(\tilde{w}, 0,0 ; \tilde{R})|\tilde{w}|^{2}$,

(19) $D^{2} c(w, z, \infty ; R)=D^{2} c(\tilde{w}, \tilde{z}, 0 ; \tilde{R})|\tilde{w} \tilde{z}|^{2}$,

where $\tilde{\xi}=1 / \xi(\xi=w, z)$ and $\tilde{R}=\{\xi=1 / \xi ; \xi \in R\}$. In fact, Lemma 7 and (12) yield (18). The unicity of $(K, L)$ shows that $K(w, \bar{z} ; R)=K(\tilde{w}, \overline{\bar{z}} ; \tilde{R}) \tilde{w} \bar{z}$ and $L(w, z ; R)=-L(\tilde{w}, \tilde{z} ; \tilde{R}) \tilde{w} \tilde{z}$, and hence (12) gives that

$$
\begin{aligned}
& D^{2} c(w, z, \infty ; R)=2 \operatorname{Re}[\{L(w, z ; R) \overline{g(w ; R)}-\overline{K(w, \bar{z} ; R)} \phi(w ; R)\} \overline{\phi(z ; R)} \\
& \text { - }\{L(w, z ; R) \overline{\phi(w ; R)}-\overline{K(w, \bar{z} ; R)} g(w, R)\} \overline{g(z ; R)}] \\
& =2 \operatorname{Re}[\{L(\tilde{w}, \tilde{z} ; \tilde{R}) \overline{K(\tilde{w}, \overline{0} ; \tilde{R})}-\overline{K(\tilde{w}, \tilde{z} ; \tilde{R})} L(\tilde{w}, 0 ; \tilde{R})\} \overline{L(\tilde{z}, 0 ; \tilde{R})} \\
& -\{L(\tilde{w}, \tilde{z} ; \tilde{R}) \overline{L(\tilde{w}, 0 ; \tilde{R})}-\overline{K(\tilde{w}, \bar{z} ; \tilde{R})} K(\tilde{w}, \overline{0} ; \tilde{R})\} \overline{K(\tilde{z}, \overline{0} ; \tilde{R})}]|\tilde{w} \tilde{z}|^{2} \\
& =D^{2} c(\tilde{w}, \tilde{z}, 0 ; \tilde{R})|\tilde{w} \tilde{z}|^{2},
\end{aligned}
$$


which implies (19).

Without loss of generality, we may assume that $\Omega-\Gamma \ni 0$. Let $w_{s}^{*}$ $(0 \leq s \leq|\tilde{\Gamma}|)$ be the arc-length representation of $\tilde{\Gamma}=\{\tilde{\xi} ; \xi \in \Gamma\}$ such that $w_{0}^{*}=\tilde{w}_{0}$, and let $s=s(t)$ be the function on $[0,|\Gamma|]$ defined by $w_{s}^{*}=\tilde{w}_{t}$. Then $d s / d t=\left|w_{s}^{*}\right|^{2}$. Using (18) with $R=\Omega_{t}, w=w_{u}(u>t)$, we see, by Theorem 8, that $\lim _{u \downarrow t} D c\left(w_{u}, \infty ; \Omega_{t}\right)\left(=D c\left(w_{t}, \infty ; \Omega_{t}\right)\right)$ exists and

$$
D c\left(w_{t}, \infty ; \Omega_{t}\right)=D K\left(w_{s}^{*}, 0,0 ; \Omega_{s}^{*}\right)\left|w_{s}^{*}\right|^{2}\left(\Omega_{s}^{*}=\tilde{\Omega}_{t}\right) .
$$

Since $s=s(t)$ is continuous on $[0,|\Gamma|], D c\left(w_{t}, \infty ; \Omega_{t}\right)$ is also continuous on $[0,|\Gamma|]$. By Lemma 7 , Theorem 8 and (20), we have

$$
\begin{aligned}
\frac{\partial c}{\partial t}\left(\infty ; \Omega_{t}\right) & =\frac{\partial K}{\partial s}\left(0, \overline{0} ; \Omega_{s}^{*}\right) \frac{d s}{d t} \\
& =\frac{1}{4} D K\left(w_{s}^{*}, 0,0 ; \Omega_{s}^{*}\right)\left|w_{s}^{*}\right|^{2}=\frac{1}{4} D c\left(w_{t}, \infty ; \Omega_{t}\right) .
\end{aligned}
$$

Thus (5) holds. Using (19) with $R=\Omega_{t}, w=w_{u}(u>t)$, we have

$$
D^{2} c\left(w_{t}, z, \infty ; \Omega_{t}\right)=D^{2} c\left(w_{s}^{*}, \tilde{z}, 0 ; \Omega_{s}^{*}\right)\left|w_{s}^{*} \tilde{z}\right|^{2} .
$$

By Theorem 8 and (19), we obtain

$$
\begin{aligned}
\frac{\partial c}{\partial t}\left(z, \infty ; \Omega_{t}\right) & =\frac{\partial}{\partial s} D K\left(\tilde{z}, 0,0 ; \Omega_{s}^{*}\right) \frac{d s}{d t}|\tilde{z}|^{2} \\
& =\left(\frac{\partial}{\partial s}\left\{\left.L\left(\tilde{z}, 0 ; \Omega_{s}^{*}\right)\right|^{2}-\left|K\left(\tilde{z}, \overline{0} ; \Omega_{s}^{*}\right)\right|^{2}\right\}\right)\left|w_{s}^{*} \tilde{z}\right|^{2} \\
& =\frac{1}{4} D^{2} c\left(w_{s}^{*}, \tilde{z}, 0 ; \Omega_{s}^{*}\right)\left|w_{s}^{*} \tilde{z}\right|^{2}=\frac{1}{4} D^{2} c\left(w_{t}, z, \infty ; \Omega_{t}\right) .
\end{aligned}
$$

Thus (6) holds. This completes the deduction of Theorem 1.

Our method of the proof of Theorem 8 requires us to distinguish the following four cases:

Case I. $\Omega$ is bounded by a finite number of analytic Jordan curves,

$$
\Gamma=[0,1], \Omega \supset \Gamma \text { and } t=1 \text {. }
$$

Case II. $0<t \leq|\Gamma|$.

Case III. $\Omega \supset \Gamma$ and $t=0$.

Case IV. $w_{0} \in \partial \Omega$ and $t=0$.

The proof will be given in $\S \S 4-7$. Finally we give the proof of Proposition 2. A simple calculation shows that $D c(z, \zeta ; \Omega)=D c(\zeta, z ; \Omega)$ and $D^{2} c(w, z, \zeta ; \Omega)$ is invariant for any permutation of a triple $(w, z, \zeta)$ of distinct numbers in $\Omega$. Let $h$ be a conformal mapping from $\Omega$ onto $\Omega^{*}$. 
If $\Omega \ni \infty, \Omega^{*} \ni \infty$ and $h(\infty)=\infty$, then the unicity of $(K, L)$ shows that

$$
\begin{aligned}
K(z, \bar{\zeta} ; \Omega) & =K\left(h(z), \overline{h(\zeta)} ; \Omega^{*}\right) \sqrt{h^{\prime}(z)} \sqrt{\sqrt{h^{\prime}(\zeta)}}, \\
L(z, \zeta ; \Omega) & =L\left(h(z), h(\zeta) ; \Omega^{*}\right) \sqrt{h^{\prime}(z)} \sqrt{h^{\prime}(\zeta)}(z, \zeta \in \Omega-\{\infty\}, z \neq \zeta) .
\end{aligned}
$$

Note that $h^{\prime}$ is single-valued and that the values $\sqrt{h^{\prime}(z)} \sqrt{\overline{h^{\prime}(\zeta)}}, \sqrt{h^{\prime}(z)} \sqrt{h^{\prime}(\zeta)}$ are determined independent of the choice of the branch of $\sqrt{ }$. Thus a simple calculation shows that, for three distinct numbers $w, z, \zeta \in \Omega-\{\infty\}$,

$$
D^{2} c(w, z, \zeta ; \Omega)=D^{2} c\left(h(w), h(z), h(\zeta) ; \Omega^{*}\right)\left|h^{\prime}(w) h^{\prime}(z) h^{\prime}(\zeta)\right| .
$$

This equality holds in the case where $\zeta=h(\zeta)=\infty$, and in the case where $\Omega \nexists \infty, \Omega^{*} \nexists \infty$, also. Equality (19) is equivalent to (21) in the case where $\zeta=\infty$ and $h(\xi)=1 / \xi$. Since any conformal mapping $h$ is expressed in the form $h(\xi)=h^{*}((1 / \xi)+a)$ with $a \in \mathbf{C}$ and a conformal mapping $h^{*}$ such that $h^{*}(\infty)=\infty$, we have (21) for any $\zeta \in \Omega$. Since $D c(w, z, \zeta ; \Omega)$ is invariant for any permutation of $(w, z, \zeta)$, we consequently see that (21) holds for any triple $(w, z, \zeta)$ of distinct numbers in $\Omega$. This completes the proof of Proposition 2.

\section{§4. Proof of Case I}

In this section, we prove Theorem 8 in the case $I$. Since $\Gamma=[0,1]$, the function $w_{t}=t$ is the arc-length representation of $\Gamma$. We write $\Omega_{t}=$ $\Omega-[0, t](t \geq 0)$. For a domain $R \ni \infty$, let $q(z ; R)$ denote the conformal mapping from $R$ onto $\overline{\mathbf{D}}^{c}$ such that $q(\infty ; R)=\infty, q^{\prime}(\infty ; R)=\lim _{z \rightarrow \infty} q(z ; R) / z$ $>0$. We divide the proof into three steps.

First step. We show that $\partial K\left(z, \bar{\zeta} ; \Omega_{t}\right) / \partial t$ exists at $t=1$.

Let $d_{0}=\min \{1 / 2$, (the distance between $\partial \Omega$ and $\left.[0,1])\right\}$. Given $1 / 2 \leq$ $u \leq 1+d_{0}$, we take $\iota_{u}(w)=w / u$. Then the boundary of $\iota_{u}\left(\Omega_{u}\right)$ consists of $\partial \iota_{u}(\Omega)$ and $\partial[0,1]^{c}$. We write by $J_{u}(\cdot, \cdot)=J\left(\cdot, \cdot ; \Omega_{u}\right), J_{u}^{*}(\cdot, \cdot)=J\left(\cdot, \cdot ; \iota_{u}\left(\Omega_{u}\right)\right)$ $(J=K, L)$. We write also $J=J_{1}(J=K, L)$. Equality (4) and the reflection principle show that there exists $0<\varepsilon_{0}<d_{0}$ such that $J_{u}(z, \cdot), J_{u}^{*}(z, \cdot)$, $\left(J=K, L ;|u-1|<\varepsilon_{0}\right)$ are analytic in a neighborhood of $\partial \Omega$ as a function of $z$. We have

$$
\begin{array}{r}
K_{u}(z, \bar{\zeta})=K_{u}^{*}\left(\iota_{u}(z), \overline{\iota_{u}(\bar{\zeta})}\right) \sqrt{\iota_{u}^{\prime}(z)} \overline{\sqrt{\iota_{u}^{\prime}(\zeta)}}=K_{u}^{*}(z / u, \overline{\zeta / u}) / u \\
=K_{u}^{*}(z, \bar{\zeta})-(u-1)\left\{K_{u}^{*}(z, \bar{\zeta})+z \frac{\partial K_{u}^{*}}{\partial z}(z, \bar{\zeta})+\bar{\zeta} \frac{\overline{\partial K_{u}^{*}}}{\partial \zeta}(\zeta, \bar{z})\right\} \\
+o(|u-1|)
\end{array}
$$




$$
\begin{aligned}
=K_{u}^{*}(z, \zeta)-(u-1)\left\{K(z, \zeta)+z \frac{\partial K}{\partial z}(z, \bar{\zeta})+\overline{\zeta \frac{\partial K}{\partial \zeta}(\zeta, \bar{z})}\right\} & \\
& +o(|u-1|) .
\end{aligned}
$$

The reproducing property yields that

$$
\begin{aligned}
K_{u}^{*}(z, \bar{\zeta}) & =\frac{1}{2 \pi} \int_{\partial \Omega_{1}} K_{u}^{*}(w, \bar{\zeta}) \overline{K(w, \bar{z})}|d w|=\frac{1}{2 \pi} \int_{\partial \Omega \cup \partial[0,1] c} \\
= & \frac{u}{2 \pi} \int_{\partial \iota_{u}(\Omega)} K_{u}^{*}(u w, \bar{\zeta}) \overline{K(u w, \bar{z})}|d w|+\frac{1}{2 \pi} \int_{\partial[0,1] c} K_{u}^{*}(w, \bar{\zeta}) \overline{K(w, \bar{z})|d w|} \\
= & \frac{1}{2 \pi} \int_{\partial \iota_{u}(\Omega,)} K_{u}^{*}(w, \bar{\zeta}) \overline{K(w, \bar{z}) \mid} d w \mid+\frac{u-1}{2 \pi} \int_{\partial \iota_{u}(\Omega)}\left\{K_{u}^{*}(u w, \bar{\zeta}) \overline{K(u w, \bar{z})}\right. \\
& \left.+w \frac{\partial K_{u}^{*}}{\partial w}(w, \bar{\zeta}) \overline{K(w, \bar{z})}+K_{u}^{*}(w, \bar{\zeta}) \bar{w} \frac{\partial K}{\partial w}(w, \bar{z})\right\}|d w|+o(|u-1|) \\
= & K(z, \bar{\zeta})+\frac{u-1}{2 \pi} \int_{\partial \Omega}\left\{K(w, \bar{\zeta}) \overline{K(w, \bar{z})}+w \frac{\partial K}{\partial w}(w, \bar{\zeta}) \overline{K(w, \bar{z})}\right. \\
& \left.+K(w, \bar{\zeta}) \bar{w} \frac{\overline{\partial K}}{\partial w}(w, \bar{z})\right\}|d w|+o(|u-1|) .
\end{aligned}
$$

Let $\lambda_{\delta}=\partial\left\{[\delta, 1-\delta] \cup \overline{D(0, \delta)} \cup \overline{D(1, \delta)\}^{c}}\left(0<\delta<d_{0}\right)\right.$. Then, combined with (4), this yields that

$$
\begin{aligned}
& \lim _{u \rightarrow 1} \frac{1}{u-1}\left\{K_{u}^{*}(z, \bar{\zeta})-K(z, \bar{\zeta})\right\}=-\frac{1}{2 \pi} \int_{\partial \Omega}\{K(w, \bar{\zeta}) \overline{K(w, \bar{z})} \\
& +w \frac{\partial K}{\partial w}(w, \zeta) \overline{K(w, \bar{z})}+K(w, \zeta) \bar{w} \frac{\overline{\partial K}(w, \bar{z})}{\partial w}|d w| \\
& =\frac{1}{2 \pi i} \int_{\partial \Omega}\left\{K(w, \bar{\zeta}) L(w, z)+w \frac{\partial K}{\partial w}(w, \bar{\zeta}) L(w, z)\right\} d w \\
& +\overline{\frac{1}{2 \pi i} \int_{\partial \Omega} L(w, \zeta) w \frac{\partial K}{\partial w}(w, \bar{z}) d w}=K(z, \bar{\zeta})+z \frac{\partial K}{\partial z}(z, \zeta)
\end{aligned}
$$

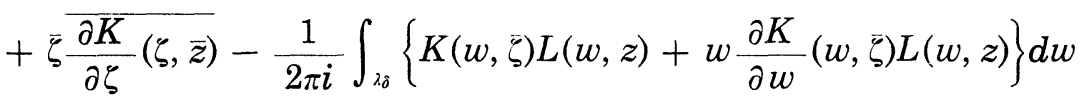

$$
\begin{aligned}
& -\overline{\frac{1}{2 \pi i} \int_{\lambda_{\hat{o}}} L(w, \zeta) w \frac{\partial K}{\partial w}(w, \bar{z}) d w} \quad\left(0<\delta<d_{0}\right)
\end{aligned}
$$

where, for the integrals on $\lambda_{\delta}$, the orientation of $d w$ is chosen so that $\{[\delta, 1-\delta] \cup \overline{D(0, \delta)} \cup \overline{D(1, \delta)}\}^{c}$ lies to the left. By (22), we have

$$
\frac{\partial K}{\partial t}\left(z, \bar{\zeta} ; \Omega_{1}\right)=\left.\frac{\partial K}{\partial t}\left(z, \bar{\zeta} ; \Omega_{t}\right)\right|_{t=1}=T(\delta) \quad\left(0<\delta<d_{0}\right)
$$


with

$$
\begin{aligned}
T(\delta)= & -\frac{1}{2 \pi i} \int_{\lambda_{\delta}}\left\{K(w, \bar{\zeta}) L(w, z)+w \frac{\partial K}{\partial w}(w, \bar{\zeta}) L(w, z)\right\} d w \\
& -\frac{1}{2 \pi i} \int_{\lambda_{\delta}} L(w, \zeta) w \frac{\partial K}{\partial w}(w, \bar{z}) d w
\end{aligned}
$$

Thus $\partial K\left(z, \bar{\zeta} ; \Omega_{1}\right) / \partial t$ exists.

Second step. We show that $D K\left(w_{1}, z, \zeta ; \Omega_{1}\right)$ exists and (17) holds at $t=1$.

Since $\partial K\left(z, \bar{\zeta} ; \Omega_{1}\right) / \partial t$ is independent of $\delta$, we have

$$
\frac{\partial K}{\partial t}\left(z, \bar{\zeta} ; \Omega_{1}\right)=\lim _{\delta \downarrow 0} T(\delta) \text {. }
$$

Express $T(\delta)$ as a summation of $T_{k}(\delta)(k=1, \cdots, 5)$ which are defined by

$$
\begin{aligned}
& T_{1}(\delta)=-\frac{1}{2 \pi i} \int_{\hat{o}}^{1-\delta}\left\{K(x+i 0, \bar{\zeta}) L(x+i 0, z)+x \frac{\partial K}{\partial w}(x+i 0, \bar{\zeta}) L(x+i 0, z)\right\} d x \\
& -\overline{\frac{1}{2 \pi i} \int_{\hat{o}}^{1-\hat{o}} L(x+i 0, \zeta) x \frac{\partial K}{\partial w}(x+i 0, \bar{z}) d x}, \\
& T_{2}(\delta)=\frac{1}{2 \pi i} \int_{\bar{o}}^{1-\delta}\left\{K(x-i 0, \bar{\zeta}) L(x-i 0, z)+x \frac{\partial K}{\partial w}(x-i 0, \bar{\zeta}) L(x-i 0, z)\right\} d x \\
& +\frac{1}{2 \pi i} \int_{j}^{1-\tilde{o}} L(x-i 0, \zeta) x \frac{\partial K}{\partial w}(x-i 0, \bar{z}) d x, \\
& T_{3}(\delta)=-\frac{1}{2 \pi i} \int_{S(1, \hat{o})} w \frac{\partial K}{\partial w}(w, \ddot{\zeta}) L(w, z) d w, \\
& T_{4}(\delta)=-\frac{1}{2 \pi i} \int_{S(1, \bar{o})} L(w, \zeta) w \frac{\partial K}{\partial w}(w, \bar{z}) d w \\
& T_{5}(\delta)=-\frac{1}{2 \pi i} \int_{S(0, \hat{o}) \cup S(1, \bar{o})} K(w, \bar{\zeta}) L(w, z) d w \\
& -\frac{1}{2 \pi i} \int_{S(0, \hat{o})} w \frac{\partial K}{\partial w}(w, \bar{\zeta}) L(w, z) d w \\
& -\overline{\frac{1}{2 \pi i}} \int_{S(0, \hat{o})} L(w, \zeta) w \frac{\partial K}{\partial w}(w, \bar{z}) d w .
\end{aligned}
$$

The behaviour of $K(z, \bar{\zeta}), L(z, \zeta)$ near $z=1$ is as follows. Taking

$$
q(z)=q\left(z ;[0,1]^{c}\right)=2\left\{\left(z-\frac{1}{2}\right)+\sqrt{\left(z-\frac{1}{2}\right)^{2}-\frac{1}{4}}\right\}
$$


we put $R=q\left(\Omega_{1}\right)$. Note that $q(1)=1$ and $K(\xi, \overline{q(\zeta)} ; R), L(\xi, q(\zeta) ; R)$ are analytic across $S(0,1)$ as a function of $\xi$. Since

$$
\begin{aligned}
& q(z)=1+2 \sqrt{z-1}+O(|z-1|), \\
& \sqrt{q^{\prime}(z)}=(z-1)^{-1 / 4}+(z-1)^{1 / 4}+O\left(|z-1|^{3 / 4}\right) \quad(z \rightarrow 1),
\end{aligned}
$$

we have

$$
\begin{aligned}
K(z, \bar{\zeta})=K(q(z), \overline{q(\zeta)} ; R) \sqrt{q^{\prime}(z)} \overline{\sqrt{q^{\prime}(\zeta)}} \\
=\left\{K(1, \overline{q(\zeta)} ; R)+2 \sqrt{z-1} \frac{\partial K}{\partial \xi}(1, \overline{q(\zeta)} ; R)+O(|z-1|)\right\} \\
\left.\quad \times\{z-1)^{-1 / 4}+(z-1)^{1 / 4}+O\left(|z-1|^{3 / 4}\right)\right\} \overline{\sqrt{q^{\prime}(\zeta)}} \\
=\left\{(z-1)^{-1 / 4} K(1, \overline{q(\zeta)} ; R)+(z-1)^{1 / 4} A_{\zeta}+O\left(|z-1|^{3 / 4}\right)\right\} \sqrt{\sqrt{q^{\prime}(\zeta)}} \quad(z \rightarrow 1)
\end{aligned}
$$

and

$$
\begin{aligned}
\frac{\partial K}{\partial z}(z, \bar{\zeta})= & -\frac{1}{4}(z-1)^{-5 / 4} K(1, \overline{q(\zeta)} ; R) \\
& \left.+\frac{1}{4}(z-1)^{-3 / 4} A_{\zeta}+O\left(|z-1|^{-1 / 4}\right)\right\} \sqrt{q^{\prime}(\zeta)} \quad(z \rightarrow 1)
\end{aligned}
$$

where, in general,

$$
\begin{aligned}
& A_{\sigma}=K(1, \overline{q(\sigma)} ; R)+2 \frac{\partial K}{\partial \xi}(1, \overline{q(\sigma)} ; R), \\
& B_{\sigma}=L(1, q(\sigma) ; R)+2 \frac{\partial L}{\partial \xi}(1, q(\sigma) ; R) .
\end{aligned}
$$

In the same manner,

$$
\begin{array}{r}
L(z, \zeta)=\left\{(z-1)^{-1 / 4} L(1, q(\zeta) ; R)+(z-1)^{1 / 4} B_{\zeta}+O\left(|z-1|^{3 / 4}\right)\right\} \sqrt{ } \overline{q^{\prime}(\zeta)} \\
(z \rightarrow 1)
\end{array}
$$

and

$$
\begin{aligned}
\frac{\partial L}{\partial z}(z, \zeta)= & \left\{-\frac{1}{4}(z-1)^{-5 / 4} L(1, q(\zeta) ; R)\right. \\
& \left.+\frac{1}{4}(z-1)^{-3 / 4} B_{\zeta}+O\left(|z-1|^{-1 / 4}\right)\right\} \sqrt{q^{\prime}(\zeta)} \quad(z \rightarrow 1)
\end{aligned}
$$

The behaviour of $K(z, \bar{\zeta}), L(z, \zeta)$ near $z=0$ is analogous. We have

$$
J(z, \cdot)=O\left(|z|^{-1 / 4}\right), \frac{\partial J}{\partial z}(z, \cdot)=O\left(|z|^{-5 / 4}\right) \quad(z \rightarrow 0, J=K, L) .
$$

We now return to the study of $T_{k}(\delta)(k=1, \cdots, 5)$. We have 


$$
\begin{aligned}
& T_{1}(\delta)=-\frac{1}{2 \pi i} \int_{0}^{1-\delta}+o(1) \\
& =-\frac{1}{2 \pi} \int_{0}^{1-\delta}\left\{K(x+i 0, \bar{\zeta}) \overline{K(x+i 0, \bar{z})}+x \frac{\partial K}{\partial x}(x+i 0, \bar{\zeta}) \overline{K(x+i 0, \bar{z})}\right. \\
& \left.+K(x+i 0, \bar{\zeta}) x \overline{\frac{\partial K}{\partial x}(x+i 0, \bar{z})}\right\} d x+o(1) \\
& =-\frac{1}{2 \pi} \int_{0}^{1-\bar{\delta}} \frac{\partial}{\partial x}\{x K(x+i 0, \bar{\zeta}) \overline{K(x+i 0, \bar{z})}\} d x+o(1) \\
& =-\frac{1}{2 \pi} K(1-\delta+i 0, \bar{\zeta}) \overline{K(1-\delta+i 0, \bar{z})}+o(1) \\
& =-\frac{1}{2 \pi}\left\{\delta^{-1 / 4} e^{-i \pi / 4} K(1, \overline{q(\zeta)} ; R)+\delta^{1 / 4} e^{i \pi / 4} A_{\zeta}+O\left(\delta^{3 / 4}\right)\right\} \\
& \times\left\{\delta^{-1 / 4} e^{i \pi / 4} \overline{K(1, \overline{q(z)} ; R)}+\delta^{1 / 4} e^{-i_{\pi / 4}} \bar{A}_{z}+O\left(\delta^{3 / 4}\right)\right\} \sqrt{q^{\prime}(z)} \overline{\sqrt{q^{\prime}(\zeta)}}+o(1) \\
& =\left[-\frac{1}{2 \pi} \delta^{-1 / 2} K(1, \overline{q(\zeta)} ; R) \overline{K(1, \overline{q(z)} ; R)}\right. \\
& \left.+\frac{i}{2 \pi}\left\{K(1, \overline{q(\zeta)} ; R) \bar{A}_{z}-\overline{K(1, \overline{q(z)} ; R)} A_{\zeta}\right\}\right] \sqrt{q^{\prime}(z)} \overline{\sqrt{q^{\prime}(\zeta)}}+o(1) .
\end{aligned}
$$

In the same manner,

$$
\begin{aligned}
T_{2}(\delta)= & {\left[-\frac{1}{2 \pi} \delta^{-1 / 2} K(1, \overline{q(\zeta)} ; R) \overline{K(1, \overline{q(z)} ; R)}\right.} \\
& \left.-\frac{i}{2 \pi}\left\{K(1, \overline{q(\zeta)} ; R) \bar{A}_{z}-\overline{K(1, \overline{q(z)} ; R)} A_{\zeta}\right\}\right] \sqrt{q^{\prime}(z)} \overline{\sqrt{q^{\prime}(\zeta)}}+o(1) .
\end{aligned}
$$

We have

$$
\begin{aligned}
T_{3}(\delta)= & -\frac{1}{2 \pi i} \int_{S(1, \hat{o})} \frac{\partial K}{\partial w}(w, \zeta) L(w, z) d w+o(1) \\
= & -\frac{1}{2 \pi i} \int_{S(0, \delta)}\left\{-\frac{1}{4} w^{-5 / 4} K(1, \overline{q(\zeta)} ; R)+\frac{1}{4} w^{-3 / 4} A_{\zeta}+O\left(|w|^{-1 / 4}\right)\right\} \\
& \times\left\{w^{-1 / 4} L(1, q(z) ; R)+w^{1 / 4} B_{z}+O\left(|w|^{3 / 4}\right)\right\} d w \sqrt{q^{\prime}(z)} \overline{\sqrt{q^{\prime}(\zeta)}}+o(1) \\
= & -\frac{1}{2 \pi i} \int_{S(0, o)}\left[-\frac{1}{4} w^{-3 / 2} K(1, \overline{q(\zeta)} ; R) L(1, q(z) ; R)\right. \\
& \left.-\frac{1}{4 w}\left\{K(1, \overline{q(\zeta)} ; R) B_{z}-L(1, q(z) ; R) A_{\zeta}\right\}\right] d w \sqrt{q^{\prime}(z)} \overline{\sqrt{q^{\prime}(\zeta)}}+o(1) \\
= & -\frac{1}{2 \pi} \delta^{-1 / 2} K(1, \overline{q(\zeta)} ; R) L(1, q(z) ; R) \\
& \left.-\frac{1}{4}\left\{K(1, \overline{q(\zeta)} ; R) B_{z}-L(1, q(z) ; R) A_{\zeta}\right\}\right] \sqrt{q^{\prime}(z)} \overline{\sqrt{q^{\prime}(\zeta)}}+o(1)
\end{aligned}
$$


In the same manner,

$$
\begin{aligned}
T_{4}(\delta)= & {\left[-\frac{1}{2 \pi} \delta^{-1 / 2} \overline{L(1, q(\zeta) ; R) K(1, \overline{q(z)} ; R)}\right.} \\
& \left.-\frac{1}{4}\left\{\overline{K(1, \overline{q(z)} ; R) B_{\zeta}}-\overline{L(1, q(\zeta) ; R) A_{z}}\right\}\right] \sqrt{q^{\prime}(z)} \overline{\sqrt{q^{\prime}(\zeta)}}+o(1) .
\end{aligned}
$$

Evidently, $\quad T_{5}(\delta)=o(1)$. Using $L(1, q(\sigma) ; R)=-\overline{K(1, \overline{q(\sigma)} ; R)} \quad(\sigma=z, \zeta)$, we obtain

$$
\begin{aligned}
& T(\delta)=\sum_{k=1}^{5} T_{k}(\delta)=\left[-\frac{1}{2 \pi} \delta^{-1 / 2} K(1, \overline{q(\zeta)} ; R) \overline{K(1, \overline{q(z)} ; R)}\right. \\
& +\frac{i}{2 \pi}\left\{K(1, \overline{q(\zeta)} ; R) \bar{A}_{z}-\overline{K(1, \overline{q(z)} ; R)} A_{\zeta}\right\} \\
& -\frac{1}{2 \pi} \delta^{-1 / 2} K(1, \overline{q(\zeta)} ; R) \bar{K}(1, \overline{q(z)} ; R) \\
& -\frac{i}{2 \pi}\left\{K(1, \overline{q(\zeta)} ; R) \bar{A}_{z}-\overline{K(1, \overline{q(z)} ; R)} A_{\xi}\right\} \\
& -\frac{1}{2 \pi} \delta^{-1 / 2} K(1, \overline{q(\zeta)} ; R) L(1, q(z) ; R) \\
& -\frac{1}{4}\left\{K(1, \overline{q(\zeta)} ; R) B_{z}-L(1, q(z) ; R) A_{\zeta}\right\} \\
& -\frac{1}{2 \pi} \delta^{-1 / 2} \overline{L(1, q(\zeta) ; R) K(1, \overline{q(z)} ; R)} \\
& \left.-\frac{1}{4}\left\{\overline{K(1, \overline{q(z)} ; R) B_{\zeta}}-\overline{L(1, q(\zeta) ; R) A_{z}}\right\}\right] \sqrt{q^{\prime}(z)} \overline{\sqrt{q^{\prime}(\zeta)}}+o(1) \\
& =\frac{1}{4}\left[-K(1, \overline{q(\zeta)} ; R) B_{z}+L(1, q(z) ; R) A_{\zeta}\right. \\
& \left.-\overline{K(1, \overline{q(z)} ; R) B_{\zeta}}+\overline{L(1, q(\zeta) ; R) A_{z}}\right] \sqrt{q^{\prime}(z)} \sqrt{q^{\prime}(\zeta)}+o(1) \\
& =\frac{1}{4}\left[L(1, q(z) ; R) \bar{B}_{\zeta}+\overline{L(1, q(\zeta) ; R)} B_{z}\right. \\
& \left.-\overline{K(1, \overline{q(z)} ; R)} A_{\zeta}-K(1, \overline{q(\zeta)} ; R) \bar{A}_{z}\right] \sqrt{q^{\prime}(z)} \overline{\sqrt{q^{\prime}(\zeta)}}+o(1) \\
& \text { ( = } T_{0} / 4+o(1), \text { say) }
\end{aligned}
$$

which shows that

$$
\frac{\partial K}{\partial t}\left(z, \bar{\zeta} ; \Omega_{1}\right)=\lim _{\bar{o} \downarrow 0} T(\delta)=T_{0} / 4
$$

By (4), we have 


$$
L(1, q(z) ; R) \overline{L(1, q(\zeta) ; R)}=\overline{K(1, \overline{q(z)} ; R)} K(1, \overline{q(\zeta)} ; R)
$$

and hence

$$
\begin{aligned}
& D K\left(1, z, \zeta ; \Omega_{1}\right)=\lim _{u \downarrow 1}\{L(u, z) \overline{L(u, \zeta)}-\overline{K(u, \bar{z})} K(u, \zeta)\} \\
& =\lim _{u \downarrow 1}\left[\left\{(u-1)^{-1 / 4} L(1, q(z) ; R)+(u-1)^{1 / 4} B_{z}+O\left((u-1)^{3 / 4}\right)\right\}\right. \\
& \times\left\{(u-1)^{-1 / 4} \overline{L(1, q(\zeta) ; R)}+(u-1)^{1 / 4} \bar{B}_{\zeta}+O\left((u-1)^{3 / 4}\right)\right\} \\
& -\left\{(u-1)^{-1 / 4} \bar{K}\left(\overline{1, \overline{q(z)} ; R)}+(u-1)^{1 / 4} \bar{A}_{z}+O\left((u-1)^{3 / 4}\right)\right\}\right. \\
& \times\left\{(u-1)^{-1 / 4} K(1, \overline{q(\zeta)} ; R)+(u-1)^{1 / 4} A_{\zeta}+O\left((u-1)^{3 / 4}\right)\right\} \\
& \left.\times \sqrt{q^{\prime}(z)} \overline{\sqrt{q^{\prime}(\zeta)}}\right] \\
& =\left\{L(1, q(z) ; R) \bar{B}_{\zeta}+\overline{L(1, q(\zeta) ; R)} B_{z}\right. \\
& \left.-\overline{K(1, \overline{q(z)} ; R)} A_{\zeta}-K(1, \overline{q(\zeta)} ; R) \bar{A}_{z}\right\} \sqrt{q^{\prime}(z)} \overline{\sqrt{q^{\prime}(\zeta)}}=T_{0}^{\prime} .
\end{aligned}
$$

Thus $D K\left(1, z, \zeta ; \Omega_{1}\right)$ exists and (17) holds.

Final step. We show that $\partial L\left(z, \zeta ; \Omega_{1}\right) / \partial t, D L\left(w_{1}, z, \zeta ; \Omega_{1}\right)$ exist and (17) holds with $K$ replaced by $L$.

We may assume that $z \neq \zeta$. For $1<u<1+d_{0}$, we have

$$
\begin{aligned}
& L_{u}(z, \zeta)-L(z, \zeta)=\frac{1}{2 \pi i} \int_{\partial \Omega_{u}} L_{u}(w, \zeta) L(w, z) d w \\
& \quad=-\frac{1}{2 \pi i} \int_{\partial \Omega_{1}} K_{u}(w, \bar{\zeta}) K(w, \bar{z}) d w+\frac{1}{2 \pi i} \int_{\partial[1, u] c} L_{u}(w, \zeta) L(w, z) d w \\
& \quad=T_{1}^{*}+T_{2}^{*}, \text { say. }
\end{aligned}
$$

Taking

$$
q_{u}(z)=q\left(z ;[0, u]^{c}\right)=\frac{2}{u}\left\{z-\frac{u}{2}+\sqrt{\left(z-\frac{u}{2}\right)^{2}-\left(\frac{u}{2}\right)^{2}}\right\},
$$

we put $R_{u}=q_{u}\left(\Omega_{u}\right)$. We have

$$
\begin{aligned}
& q_{u}(z)=1+2 \sqrt{z-u}+O(u-1), \\
& \sqrt{q_{u}^{\prime}(\bar{z})}=u^{-1 / 4}(z-u)^{-1 / 4}+(z-u)^{1 / 4}+O\left((u-1)^{3 / 4}\right),
\end{aligned}
$$

where $O(\cdot)$ means the uniform estimate with respect to $z \in \partial[1, u]^{c}$ when $u \downarrow 1$. Thus

$$
\begin{aligned}
K_{u}(z, \bar{\zeta})= & K\left(q_{u}(z), \overline{q_{u}(\zeta)} ; R_{u}\right) \sqrt{q_{u}^{\prime}(z)} \overline{\sqrt{q_{u}^{\prime}(\zeta)}} \\
= & \left\{K\left(1, \overline{q_{u}(\zeta)} ; R_{u}\right)+2 \sqrt{z-u} \frac{\partial K}{\partial \xi}\left(1, \overline{q_{u}(\zeta)} ; R_{u}\right)+O(u-1)\right\} \\
& \times\left\{u^{-1 / 4}(z-u)^{-1 / 4}+(z-u)^{1 / 4}+O\left((u-1)^{3 / 4}\right)\right\} \sqrt{\sqrt{q_{u}^{\prime}(\zeta)}}
\end{aligned}
$$




$$
\begin{aligned}
= & \left\{u^{-1 / 4}(z-u)^{-1 / 4} K\left(1, \overline{q_{u}(\zeta)} ; R_{u}\right)+(z-u)^{1 / 4} A_{u, \zeta}\right. \\
& \left.+O\left((u-1)^{3 / 4}\right)\right\} \sqrt{\overline{q_{u}^{\prime}(\zeta)}}\left(z \in \partial[1, u]^{c}, u \downarrow 1\right),
\end{aligned}
$$

where $\frac{\partial K}{\partial \xi}\left(1, \overline{q_{u}(\zeta)} ; R_{u}\right)=\left.\frac{\partial K}{\partial \xi}\left(\xi, \overline{q_{u}(\zeta)} ; R_{u}\right)\right|_{\xi=1}$, and, in general,

$$
\begin{aligned}
& A_{u, \sigma}=K\left(1, \overline{q_{u}(\sigma)} ; R_{u}\right)+2 u^{-1 / 4} \frac{\partial K}{\partial \xi}\left(1, \overline{q_{u}(\sigma)} ; R_{u}\right), \\
& B_{u, \sigma}=L\left(1, q_{u}(\sigma) ; R_{u}\right)+2 u^{-1 / 4} \frac{\partial L}{\partial \xi}\left(1, q_{u}(\sigma) ; R_{u}\right) .
\end{aligned}
$$

In the same manner,

$$
\begin{aligned}
L_{u}(z, \zeta)= & \left\{u^{-1 / 4}(z-u)^{-1 / 4} L\left(1, q_{u}(z) ; R_{u}\right)+(z-u)^{1 / 4} B_{u, \zeta}\right. \\
& \left.+O\left((u-1)^{3 / 4}\right)\right\} \sqrt{q_{u}^{\prime}(\zeta)} \quad\left(z \in \partial[1, u]^{\mathrm{c}}, u \downarrow 1\right) .
\end{aligned}
$$

Note that

$$
\begin{aligned}
\frac{1}{2 \pi i} \int_{\partial[1, u] c}(w-u)^{-1 / 4}(w-1)^{-1 / 4} d w & =\frac{1}{2 \pi i}\left\{\overline{\left.e^{-i \pi / 4}-e^{i \pi / 4}\right\}}\right. \\
\times \int_{0}^{u-1}(u-1-x)^{-1 / 4} x^{-1 / 4} d x & =\frac{\sqrt{u-1}}{\sqrt{2} \pi} B\left(\frac{3}{4}, \frac{3}{4}\right), \\
\frac{1}{2 \pi i} \overline{\int_{\partial[1, u] c}(w-u)^{-1 / 4}(w-1)^{1 / 4} d w} & =\frac{1}{2 \pi i}\left\{\overline{\left.e^{-i \pi / 4}-e^{i \pi / 4}\right\}}\right. \\
\times \int_{0}^{u-1}(u-1-x)^{-1 / 4} x^{1 / 4} d x & =\frac{u-1}{\sqrt{2} \pi} B\left(\frac{3}{4}, \frac{5}{4}\right)=\frac{u-1}{4}, \\
\frac{1}{2 \pi i} \int_{\hat{o}[1, u] c}(w-u)^{1 / 4}(w-1)^{-1 / 4} d w & =\frac{u-1}{2 \pi i}\left\{\overline{e^{i \pi / 4}-e^{-i \pi / 4}}\right\} B\left(\frac{5}{4}, \frac{3}{4}\right) \\
& =-\frac{u-1}{4},
\end{aligned}
$$

where the orientation of $d w$ is chosen so that $[0, u]^{c}$ lies to the left. Thus

$$
\begin{aligned}
T_{1}^{*}= & \frac{1}{2 \pi i} \int_{\partial[1, u] c} K_{u}(w, \bar{\zeta}) K(w, \bar{z}) d w \\
= & {\left[\frac{1}{2 \pi i} \int_{\partial[1, u]^{c}} \overline{\left\{u^{-1 / 4}(w-u)^{-1 / 4} K\left(1, \overline{q_{u}(\zeta)} ; R_{u}\right)+(w-u)^{1 / 4} A_{u, \zeta}\right.}\right.} \\
& \left.\frac{\left.+O\left((u-1)^{3 / 4}\right)\right\}}{+}+\frac{(w-1)^{1 / 4} A_{z}}{\left.+O\left(|w-1|^{3 / 4}\right)\right\} d w}\right] \sqrt{q^{\prime}(\boldsymbol{z})} \sqrt{q_{u}^{\prime}(\zeta)}
\end{aligned}
$$




$$
\begin{aligned}
= & {\left[u^{-1 / 4} \overline{K\left(1, \overline{q_{u}(\zeta)} ; R_{u}\right) K(1, \overline{q(z)} ; R)} \frac{1}{2 \pi i} \overline{\int_{\partial[1, u]^{c}}(w-u)^{-1 / 4}(w-1)^{-1 / 4}} d w\right.} \\
& +u^{-1 / 4} \overline{K\left(1, \overline{q_{u}(\zeta)} ; R_{u}\right) A_{z}} \frac{1}{2 \pi i} \overline{\int_{\partial[1, u]^{c}}} \overline{(w-u)^{-1 / 4}(w-1)^{1 / 4} d w} \\
& \left.+\overline{K(1, \overline{q(z)} ; R) A_{u, \zeta}} \frac{1}{2 \pi i} \overline{\int_{\partial[1, u] c}(w-u)^{1 / 4}(w-1)^{-1 / 4} d w}\right] \sqrt{q^{\prime}(z)} \sqrt{q_{u}^{\prime}(\zeta)} \\
& +o(u-1) \\
= & {\left[\frac{1}{\sqrt{2} \pi}(u-1)^{1 / 2} u^{-1 / 4} B\left(\frac{3}{4}, \frac{3}{4}\right) \overline{K\left(1, \overline{q_{u}(\zeta)} ; R_{u}\right) K(1, \overline{q(z)} ; R)}\right.} \\
& +\frac{u-1}{4}\left\{u^{-1 / 4} \overline{K\left(1, \overline{q_{u}(\zeta)} ; R_{u}\right) A_{z}}-\overline{\left.K(1, \overline{q(z)} ; R) A_{u, \zeta}\right\}}\right] \sqrt{q^{\prime}(z)} \sqrt{q_{u}^{\prime}(\zeta)} \\
& +o(u-1) .
\end{aligned}
$$

In the same manner,

$$
\begin{aligned}
T_{2}^{*}= & {\left[-\frac{1}{\sqrt{2} \pi}(u-1)^{1 / 2} u^{-1 / 4} B\left(\frac{3}{4}, \frac{3}{4}\right) L\left(1, q_{u}(\zeta) ; R_{u}\right) L(1, q(z) ; R)\right.} \\
& \left.-\frac{u-1}{4}\left\{u^{-1 / 4} L\left(1, q_{u}(\zeta) ; R_{u}\right) B_{z}-L(1, q(z) ; R) B_{u, \zeta}\right\}\right] \sqrt{q^{\prime}(z)} \sqrt{q_{u}^{\prime}(\zeta)} \\
& +o(u-1) .
\end{aligned}
$$

Since

$$
L\left(1, q_{u}(\zeta) ; R_{u}\right) L(1, q(z) ; R)=\overline{K\left(1, \overline{q_{u}(\zeta)} ; R_{u}\right) K(1, \overline{q(z)} ; R)}
$$

we have

$$
\begin{aligned}
& \lim _{u \downarrow 1} \frac{1}{u-1}\left\{L_{u}(z, \zeta)-L(z, \zeta)\right\} \\
&=\lim _{u \downarrow 1} \frac{1}{4}\left[u^{-1 / 4} \overline{K\left(1, \overline{q_{u}(\zeta)} ; R_{u}\right) A_{z}}-\overline{K(1, \overline{q(z)} ; R) A_{u, \zeta}}\right. \\
&\left.-u^{-1 / 4} L\left(1, q_{u}(\zeta) ; R_{u}\right) B_{z}+L(1, q(z) ; R) B_{u, \zeta}\right] \sqrt{q^{\prime}(z)} \sqrt{q_{u}^{\prime}(\zeta)} \\
&= \frac{1}{4} \overline{\left[K(1, \overline{q(\zeta)} ; R) A_{z}\right.}-\overline{K(1, \overline{q(z)} ; R) A_{\zeta}} \\
&\left.\quad-L(1, q(\zeta) ; R) B_{z}+L(1, q(z) ; R) B_{\zeta}\right] \sqrt{q^{\prime}(z)} \sqrt{q^{\prime}(\zeta)}=T_{0}^{*} / 4, \text { say. }
\end{aligned}
$$

Thus the right-derivative $\partial^{+} L\left(z, \zeta ; \Omega_{1}\right) / \partial t$ exists and equals $T_{0}^{*} / 4$. In the same manner, we see that the same property holds for the leftderivative. Consequently, $\partial L\left(z, \zeta ; \Omega_{1}\right) / \partial t$ exists and equals $T_{0}^{*} / 4$. Since 


$$
\begin{aligned}
& D L\left(1, z, \zeta ; \Omega_{1}\right)=\lim _{u ! 1}\{L(u, z) \overline{K(u, \bar{\zeta})}-\overline{K(u, \bar{z})} L(u, \zeta)\} \\
& =\lim _{u \downarrow 1}\left[\left\{(u-1)^{-1 / 4} L(1, q(z) ; R)+(u-1)^{1 / 4} B_{z}+O\left((u-1)^{3 / 4}\right)\right\}\right. \\
& \times\left\{(u-1)^{-1 / 4} \overline{K(1, \overline{q(\zeta)} ; R)}+(u-1)^{1 / 4} \bar{A}_{\zeta}+O\left((u-1)^{3 / 4}\right)\right\} \\
& -\left\{(u-1)^{-1 / 4} \overline{K(1, \overline{q(z)} ; R)}+(u-1)^{1 / 4} \bar{A}_{z}+O\left((u-1)^{3 / 4}\right)\right\} \\
& \left.\times\left\{(u-1)^{-1 / 4} L(1, q(\zeta) ; R)+(u-1)^{1 / 4} B_{\zeta}+O\left((u-1)^{3 / 4}\right)\right\}\right] \\
& \times \sqrt{q^{\prime}(z)} \sqrt{q^{\prime}(\zeta)} \\
& =\left[-L(1, q(\zeta) ; R) \bar{A}_{z}+L(1, q(z) ; R) \bar{A}_{\zeta}\right. \\
& \left.+\overline{K(1, \overline{q(\zeta)} ; R)} B_{z}-\overline{K(1, \overline{q(z)} ; R)} B_{\zeta}\right] \sqrt{q^{\prime}(z)} \sqrt{q^{\prime}(\zeta)} \\
& =\overline{\left[K(1, \overline{q(\zeta)} ; R) A_{z}\right.}-\overline{K(1, \overline{q(z)} ; R) A_{\zeta}} \\
& \text { - } \left.L(1, q(\zeta) ; R) B_{z}+L(1, q(z) ; R) B_{\zeta}\right] \sqrt{q^{\prime}(z)} \sqrt{q^{\prime}(\zeta)}=T_{0}^{*} .
\end{aligned}
$$

Thus $D L\left(1, z, \zeta ; \Omega_{1}\right)$ exists and $\partial L\left(z, \zeta ; \Omega_{1}\right) / \partial t=D L\left(1, z, \zeta ; \Omega_{1}\right) / 4$. This completes the proof of Theorem 8 in the case $I$.

\section{§5. Deduction of Case II from Case I}

In this section, we deduce the case II from the case I. We work only with $K$; the argument for $L$ is analogous. The arc-length representation of a closed analytic arc $\Gamma$ is denoted by $w_{t}(0 \leq t \leq|\Gamma|)$. For $-\pi<\theta \leq \pi$, let $\mathscr{L}_{\theta}$ denote the totality of closed arcs $\Gamma$ such that $\Gamma-\{0\}$ is analytic and $\Gamma$ is expressed as $e^{i \theta} x+x^{3 / 2} P(\sqrt{x}), 0 \leq x<r$ in a neighborhood of 0 with an analytic function $P$ in $D\left(0, r^{2}\right)$. For $\Gamma \in \mathscr{L}_{\theta}$, let $w_{t}(0 \leq t \leq|\Gamma|)$ denote the arc-length representation of $\Gamma$ such that $w_{0}=0$. We put $\Lambda_{t}=[-1,0] \cup \Gamma_{t}(0 \leq t \leq|\Gamma|)$. Here are some lemmas necessary for the proof of the cases II-IV.

Lemma 9. Let $\Gamma$ be a closed analytic arc and let $A$ be an arc in $\mathscr{A}$ with an endpoint $w_{0}$ such that $\Lambda \cap \Gamma=\left\{w_{0}\right\}$. Let $h$ be the conformal mapping from $\Lambda^{c}$ onto $[-1,0]^{c}$ such that $h(\infty)=\infty$ and $h(0)=0$. Then $h^{\prime}(0)$ exists, $h^{\prime}\left(w_{t}\right)$ is continuous on $t \in[0,|\Gamma|]$ and $h(\Gamma) \in \mathscr{L}_{\theta}$ for some $-\pi<\theta \leq \pi$.

To see this lemma, we may assume that $w_{0}=0$ and $\Lambda$ belongs to $\mathscr{L}_{\pi}$ in a neighborhood of 0 . Then $\Gamma \in \mathscr{L}_{\theta}$ for some $-\pi<\theta \leq \pi$. Take an analytic arc $\gamma$ passing through 0 such that $\gamma^{2} \subset \Lambda$. Then $\zeta^{2}$ is a conformal mapping from a domain $U$ bounded by $\gamma$ and a small circle $S(0, r)$ onto $D\left(0, r^{2}\right)-\Lambda$. Form an analytic function

$$
h^{*}(\zeta)=q\left(h\left(\zeta^{2}\right) ;[-1,0]^{c}\right)=2\left\{h\left(\zeta^{2}\right)+\frac{1}{2}+\sqrt{\left\{h\left(\zeta^{2}\right)+\frac{1}{2}\right\}^{2}-\frac{1}{4}}\right\}
$$


in $U$. Since $h^{*}(\gamma)$ is an arc on $S(0,1)$, the reflection principle shows that $h^{*}$ is analytic in a small disk $D\left(0, r^{\prime}\right)\left(0<r^{\prime}<r\right)$. Since $\Lambda \in \mathscr{L}_{0}, h^{*}(0)=1$ and $h^{*}$ is one-to-one, we can write $h^{*}(\zeta)=1+a \zeta \hat{h}(\zeta)$ with $a \in \mathbf{R}, a \neq 0$ and an analytic function $\hat{h}$ in $D\left(0, r^{\prime}\right)$ such that $\hat{h}(0)=1$. This shows that

$$
\begin{aligned}
h\left(\zeta^{2}\right) & =\frac{1}{4}\left\{h^{*}(\zeta)+\frac{1}{h^{*}(\zeta)}-2\right\} \\
& =\frac{1}{4}\left\{1+a \hat{h}(\zeta)+(1+a \hat{h}(\zeta))^{-1}-2\right\} \\
& =4^{-1} a^{2} \zeta^{2} \hat{h}(\zeta)^{2}\{1+a \zeta \hat{h}(\zeta)\}^{-1},
\end{aligned}
$$

and hence we can write

$$
h(z)=4^{-1} a^{2} z \hat{h}(\sqrt{z})^{2}\{1+a \sqrt{z} \hat{h}(\sqrt{z})\}^{-1} \quad\left(z \in D\left(0, r^{\prime}\right)\right)
$$

with a suitable branch of $\sqrt{ }$. Thus $h^{\prime}(0)$ exists and equals $a^{2} / 4$. Since $h^{\prime}(z)=a^{2} / 4+o(1)(z \rightarrow 0), h^{\prime}\left(w_{t}\right)$ is continuous at $t=0$, which yields that $h^{\prime}\left(w_{t}\right)$ is continuous on $[0,|\Gamma|]$. Since $\Gamma \in \mathscr{L}_{\theta}$ and $\Gamma$ is analytic, there exists an analytic function $g$ in a small disk $D\left(0, r^{\prime \prime}\right)\left(0<r^{\prime \prime}<r^{\prime}\right)$ such that $g(0)=0, g^{\prime}(0)=e^{i \theta}$ and $g\left(\left[0, r^{\prime \prime}\right]\right) \subset \Gamma$ in a neighborhood of 0 . Then $h(\Gamma)$ is expressed as

$$
4^{-1} a^{2} e^{i \theta} x\left[e^{-i \theta} \frac{g(x)}{x} \hat{h}(\sqrt{g(x)})^{2}\{1+a \sqrt{g(x)} \hat{h}(\sqrt{g(x)})\}^{-1}\right], \quad 0 \leq x<r^{\prime \prime}
$$

in a neighborhood of 0 . Substituting the variable $x$ by $4^{-1} a^{2} x$, we obtain $h(\Gamma) \in \mathscr{L}_{\theta}$.

Lemma 10. Let $\Gamma$ be a closed analytic arc and let $k_{t}$ be the conformal mapping from $\Gamma_{t}^{c}$ onto $\overline{D\left(0, r_{t}\right)^{c}}$ such that $k_{t}(\infty)=\infty,\left|k_{t}^{\prime}(\infty)\right|=1$ and $k_{t}\left(w_{t}\right)=r_{t} \quad(0<t \leq|\Gamma|)$. Then the limit $\lim _{u \downarrow t}\left\{k_{t}\left(w_{u}\right)-r_{t}\right\}\left|k_{t}^{\prime}\left(w_{u}\right)\right|\left(=a_{t}\right.$, say) exists, $a_{t}$ is continuous in $(0,|\Gamma|], a_{t}>0$ and $\lim _{t \downarrow 0} a_{t} / r_{t}=1 / 2$.

In the same manner as above, we see that $a_{t}$ exists, $a_{t}$ is continuous in $(0,|\Gamma|]$ and $a_{t}>0$. There remains to prove $\lim _{t \downarrow 0} a_{t} / r_{t}=1 / 2$. We can write

$$
\begin{aligned}
& k_{t}\left(w_{u}\right)=k_{t}\left(w_{t}\right)+b_{t} \sqrt{w_{u}-w_{t}}+O(u-t), \\
& k_{t}^{\prime}\left(w_{u}\right)=\frac{1}{2} b_{t}\left(w_{u}-w_{t}\right)^{-1 / 2}+O(1) \quad(u \downarrow t)
\end{aligned}
$$

with $b_{t} \sqrt{d w_{t} / d t}>0$. Then $a_{t}=\left|b_{t}\right|^{2} / 2$. Thus it is sufficient to show that 
$\lim _{t \downarrow 0}\left|b_{t}\right| / \sqrt{r_{t}}=1$. To do this, we may assume that $w_{0}=0$. Then $\Gamma \in \mathscr{L}_{\theta}$ for some $-\pi<\theta \leq \pi$. Let $g$ be the conformal mapping in the proof of Lemma 9 and, for a small number $t>0$, let $s=s_{0}(t)$ be the number defined by $g(s)=w_{t}$. Form a conformal mapping

$$
k_{t}^{*}(z)=k_{t} \circ g\left(z+\frac{s^{2}}{16 z}+\frac{s}{2}\right)
$$

in a small ring $D\left(0, r^{*}\right)-\overline{D(0, s / 4)}$ such that $k_{t}^{*}(s / 4)=r_{t}$ and $k_{t}^{*}(S(0, s / 4))$ $=S\left(0, r_{t}\right) ; r^{*}$ is a small number independent of $t$. The reflection principle shows that $k_{t}^{*}$ is analytic in a ring $D\left(0, r^{*}\right)-\overline{D\left(0, s^{2} /\left(16 r^{*}\right)\right)}$ with the identity $k_{t}^{*}(z)=r_{t}^{2} \overline{\left(k_{t}^{*}\left(s^{2} /(16 \bar{z})\right)\right)^{-1}}$ in $D(0, s / 4)-\overline{D\left(0, s^{2} /\left(16 r^{*}\right)\right)}$. Since $k_{t}(\zeta)$ uniformly converges to $\zeta$ on $S\left(0, r^{*}\right)$, we have

$$
\begin{aligned}
k_{t}^{* \prime}(s / 4)= & \frac{1}{2 \pi i} \int_{\partial\left\{D\left(0, r^{*}\right)-D\left(0, s^{2} /\left(16 r^{*}\right)\right)\right\}} \frac{1}{(z-(s / 4))^{2}} k_{t}^{*}(z) d z \\
= & \frac{1}{2 \pi} \int_{0}^{2 \pi} \frac{1}{\left\{r^{*} e^{i \psi}-(s / 4)\right\}^{2}} k_{t}^{*}\left(r^{*} e^{i \psi}\right) r^{*} e^{i \psi} d \psi \\
& -\frac{1}{2 \pi} \int_{0}^{2 \pi} \frac{1}{\left\{\left(s^{2} /\left(16 r^{*}\right)\right) e^{i \psi}-(s / 4)\right\}^{2}} \overline{k_{t}^{*}\left(r^{*} e^{i \psi}\right)^{-1}} \frac{r_{t}^{2} s^{2}}{16 r^{*}} e^{i \psi} d \psi \\
= & \frac{1}{2 \pi r^{*}} \int_{0}^{2 \pi} g\left(r^{*} e^{i \psi}\right) e^{-i \psi} d \psi+o(1)=1+o(1) \quad(t \downarrow 0) .
\end{aligned}
$$

Since

$$
\begin{aligned}
\left|k_{t}^{* \prime}(s / 4)\right| & =\lim _{\varepsilon \downarrow 0} \frac{1}{\varepsilon}\left|k_{t} \circ g\left\{s+4 \varepsilon^{2} / s+o\left(\varepsilon^{2}\right)\right\}-k_{t} \circ g(s)\right| \\
& =\left|b_{t}\right| \lim _{\varepsilon \downarrow 0} \frac{1}{\varepsilon}\left|g\left\{s+4 \varepsilon^{2} / s+o\left(\varepsilon^{2}\right)\right\}-g(s)\right|^{1 / 2}=2\left|b_{t} g^{\prime}(s)\right| \mid \sqrt{s},
\end{aligned}
$$

we have

$$
2\left|b_{t} g^{\prime}(s)\right| / \sqrt{s}=1+o(1) \quad(t \downarrow 0) .
$$

Since $\left|g^{\prime}(0)\right|=1, s_{0}^{\prime}(0)=1$ and $\lim _{t \downarrow 0} t / r_{t}=4$, this shows that $\lim _{t \downarrow 0}\left|b_{t}\right| / \sqrt{r_{t}}$ $=1$. Consequently, $\lim _{t \downarrow 0} a_{t} / r_{t}=1 / 2$.

Lemma 11 . Let $\Gamma \in \mathscr{L}_{0}$. Then $\gamma\left(\Lambda_{t}\right)=(1+t) / 4+o(t) \quad(t \downarrow 0)$.

Since $\Gamma \in \mathscr{L}_{0}$, the diameter of $\Lambda_{t}$ is larger than or equal to $1+t+o(t)$, and hence $\gamma\left(\Lambda_{t}\right) \geq\{1+t+o(t)\} / 4[11$, p. 9]. There exists a constant $C$ such that $\Lambda_{t}$ is contained in a rectangle $\left\{x+i y ;-1 \leq x \leq t,|y| \leq C t^{3 / 2}\right\}$ $(0 \leq t \leq|\Gamma|)$. A simple calculation yields that 


$$
\gamma\left(\left\{x+i y ;-1 \leq x \leq t,|y| \leq C t^{3 / 2}\right\}\right)=\frac{1+t}{4}+o(t)
$$

which gives that $\gamma\left(\Lambda_{t}\right) \leq(1+t) / 4+o(t)$. Thus the required equality holds.

Lemma 12. Let $\Gamma \in \mathscr{L}_{\theta},-\pi<\theta \leq \pi$. For $0 \leq s \leq 4 \gamma([-1,0] \cup \Gamma)-1$, let $t=t^{*}(s)$ be the number defined by $\gamma\left(\Lambda_{t}\right)=(1+s) / 4$. Then, for any compact set $F$ in $([-1,0] \cup \Gamma)^{c}$,

(23) $\sup _{z \in F}\left|q\left(z ; \Lambda_{t^{*}(s)}^{c}\right)-q\left(z ;[-1, s]^{c}\right)\right|=o(s) \quad(s \downarrow 0)$.

To see this, we write simply $q_{s}^{*}(z)=q\left(z ; \Lambda_{t^{*}(s)}^{c}\right), q_{s}^{0}(z)=q\left(z ;[-1, s]^{c}\right)$. Note that

$$
\begin{gathered}
q_{s}^{0}(z)=\frac{2}{s+1}\left\{z-\frac{s-1}{2}+\sqrt{\left.\left(z-\frac{s-1}{2}\right)^{2}-\left(\frac{s+1}{2}\right)^{2}\right\}},\right. \\
\frac{\partial q_{s}^{0}}{\partial s}(z)=\frac{1}{s+1} q_{s}^{0}(z) \frac{1+q_{s}^{0}(z)}{1-q_{s}^{0}(z)} .
\end{gathered}
$$

Substituting the parameter of Löwner's D. E. by a suitable one, we obtain

$$
\frac{\partial q_{s}^{*}}{\partial s}(z)=\frac{1}{s+1} q_{s}^{*}(z) \frac{1+\kappa_{s} q_{s}^{*}(z)}{1-\kappa_{s} q_{s}^{*}(z)} \quad\left(\kappa_{s}=\overline{q_{s}^{*}\left(w_{t^{*}(s)}\right)}\right) .
$$

(See (56).) There exists $0<\varepsilon_{0}<1$ such that $\left|q_{s}^{0}(z)\right| \geq 1+\varepsilon_{0},\left|q_{s}^{*}(z)\right| \geq 1+\varepsilon_{0}$ $(z \in F, 0 \leq s \leq 4 \gamma([-1,0] \cup \Gamma)-1)$. Then

$$
\begin{aligned}
& \left|\frac{\partial}{\partial s}\right| q_{s}^{*}(z)-q_{s}^{0}(z)|| \leq\left|\frac{\partial q_{s}^{*}}{\partial s}(z)-\frac{\partial q_{s}^{0}}{\partial s}(z)\right| \\
& \quad=\frac{1}{s+1}\left|q_{s}^{*}(z) \frac{1+\kappa_{s} q_{s}^{*}(z)}{1-\kappa_{s} q_{s}^{*}(z)}-q_{s}^{0}(z) \frac{1+q_{s}^{0}(z)}{1-q_{s}^{0}(z)}\right| \\
& \quad=\frac{1}{s+1} \mid\left\{q_{s}^{*}(z)-q_{s}^{0}(z)\right\} \frac{1+\kappa_{s} q_{s}^{*}(z)}{1-\kappa_{s} q_{s}^{*}(z)} \\
& \quad+q_{s}^{0}(z)\left(\frac{1+\kappa_{s} q_{s}^{*}(z)}{1-\kappa_{s} q_{s}^{*}(z)}-\frac{1+q_{s}^{0}(z)}{1-q_{s}^{0}(z)}\right) \mid \\
& \quad \leq 3 \varepsilon_{0}^{-1}\left|q_{s}^{*}(z)-q_{s}^{0}(z)\right|+4 \varepsilon_{0}^{-1} \frac{\left|\kappa_{s} q_{s}^{*}(z)-q_{s}^{0}(z)\right|}{\left|q_{s}^{*}(z)\right|-1} \\
& \leq 11 \varepsilon_{0}^{-2}\left\{\left|q_{s}^{*}(z)-q_{s}^{0}(z)\right|+\left|\kappa_{s}-1\right|\right\} .
\end{aligned}
$$

Since $q_{0}^{*}=q_{0}^{0}$, we have

$$
\begin{aligned}
\left|q_{p}^{*}(z)-q_{s}^{0}(z)\right| & \leq 11 \varepsilon_{0}^{-2} \int_{0}^{s}\left\{\left|q_{x}^{*}(z)-q_{x}^{0}(z)\right|+\left|\kappa_{x}-1\right|\right\} d x \\
& \leq 11 \varepsilon_{0}^{-2} \int_{0}^{s}\left\{\rho(x)+\left|\kappa_{x}-1\right|\right\} d x
\end{aligned}
$$


where $\rho(x)=\sup _{z \in F}\left|q_{x}^{*}(z)-q_{x}^{0}(z)\right|$. Thus

$$
\rho(s) \leq 11 \varepsilon_{0}^{-2} \int_{0}^{s}\left\{\rho(x)+\left|\kappa_{x}-1\right|\right\} d x
$$

Let $C=\max \left\{\rho(x)+\left|\kappa_{x}-1\right| ; 0 \leq x \leq 4 \gamma([-1,0] \cup T)-1\right\}$. Then (24) shows that $\rho(s) \leq 11 C \varepsilon_{0}^{-2} s$. Using (24) again, we have

$$
\rho(s) \leq 121 C \varepsilon_{0}^{-4} s^{2}+11 \varepsilon_{0}^{-2} \int_{0}^{s}\left|\kappa_{x}-1\right| d x .
$$

Since $\lim _{s \downarrow 0} \kappa_{s}=1$, this inequality gives (23).

Now we deduce the case II from the case I. Let $\Omega \in \mathscr{G}$ and let $\Gamma$ be a closed analytic arc such that $\Omega^{c} \cap \Gamma$ is at most a singleton. If $\Omega^{c} \cap \Gamma=\left\{w_{0}\right\}$, we denote by $\Lambda$ the component of $\Omega^{c}$ such that $\Lambda \cap \Gamma=\left\{w_{0}\right\}$. The condition $\Omega-\Gamma \in \mathscr{G}$ shows that $\Lambda \cup \Gamma \in \mathscr{A}$. If $\Omega^{c} \cap \Gamma=\emptyset$, we put $\Lambda=\emptyset$. We divide the proof into several steps.

First step. There exists a conformal mapping $h$ from $\Omega \cup \Lambda$ onto a domain bounded by a finite number of analytic Jordan curves. Then $h(\Lambda \cup \Gamma) \in \mathscr{A}$ and $h(\Gamma)$ is a closed analytic arc. Let $w_{s}^{*}(0 \leq s \leq|h(\Gamma)|)$ be the arc-length representation of $h(\Gamma)$ such that $w_{0}^{*}=w_{0}$, and let $s=\hat{s}(t)$ be the function defined by $w_{s}^{*}=h\left(w_{t}\right)$. Then $d s / d t=\left|h^{\prime}\left(w_{t}\right)\right|$ and $d s / d t$ is continuous in $(0,|\Gamma|]$. If the required properties are established for a domain $h(\Omega)$, an $\operatorname{arc} h(\Gamma)$ and $0<s \leq|h(\Gamma)|$, then we have, with $\Omega_{s}^{*}=$ $h(\Omega)-\left\{w_{x}^{*} ; 0 \leq x \leq s\right\}$,

$$
\begin{array}{r}
\frac{\partial K}{\partial t}\left(z, \bar{\zeta} ; \Omega_{t}\right)=\frac{\partial K}{\partial s}\left(h(z), \overline{h(\zeta)} ; \Omega_{s}^{*}\right) \frac{d s}{d t} \sqrt{h^{\prime}(z)} \overline{\sqrt{h^{\prime}(\zeta)}}, \\
D K\left(w_{t}, z, \zeta ; \Omega_{t}\right)=D K\left(w_{s}^{*}, h(z), h(\zeta) ; \Omega_{s}^{*}\right)\left|h^{\prime}\left(w_{t}\right)\right| \sqrt{h^{\prime}(z)} \overline{\sqrt{h^{\prime}(\zeta)}} \\
(0<t \leq|\Gamma|),
\end{array}
$$

which yields the required properties for $\Omega, \Gamma$ and $0<t \leq|\Gamma|$. Thus, from the beginning, we may assume that $\Omega-\Lambda$ is bounded by a finite number of analytic Jordan curves.

Second step. We show that the right-derivative $\partial^{+} K\left(z, \bar{\zeta} ; \Omega_{t}\right) / \partial t$ at $t=0$ exists if $\Lambda=[-1,0]$ and $\Gamma \in \mathscr{L}_{0}$.

Here is an equality necessary for the proof:

$$
K\left(z, \bar{\zeta} ; \Omega_{t}\right)=K(z, \bar{\zeta} ; \Omega-[0, t])+o(t) \quad(t \downarrow 0) .
$$

To see this, we form a conformal mapping $\iota_{s}^{*}(z)=\left(q_{s}^{0}\right)^{-1} \circ q_{s}^{*}(z)$ from 
$\Omega_{t^{*}(s)}$ onto a domain $\Omega_{s}^{*}$ whose boundary consists of $[-1, s]$ and $\partial \iota_{s}^{*}(\Omega \cup \Lambda)$, where $q_{s}^{*}(z)=q\left(z ; \Lambda_{t^{*}(s)}^{c}\right), \quad q_{s}^{0}(z)=q\left(z ;[-1, s]^{c}\right)$ and $t^{*}(s)$ is the number defined in Lemma 12 for $\Gamma \in \mathscr{L}_{0}$. Then

$$
\begin{aligned}
\iota_{s}^{*}(z)= & \frac{s+1}{4}\left\{q_{s}^{*}(z)+\frac{1}{q_{s}^{*}(z)}\right\}+\frac{s-1}{2} \\
= & \frac{s+1}{4}\left\{q_{s}^{0}(z)+\frac{1}{q_{s}^{0}(z)}\right\}+\frac{s-1}{2} \\
& \quad+\frac{s+1}{4}\left\{\left(q_{s}^{*}(z)-q_{s}^{0}(z)\right)+\left(\frac{1}{q_{s}^{*}(z)}-\frac{1}{q_{s}^{0}(z)}\right)\right\} \\
= & z+\frac{s+1}{4}\left(q_{s}^{*}(z)-q_{s}^{0}(z)\right)\left(1-\frac{1}{q_{s}^{*}(z) q_{s}^{0}(z)}\right)
\end{aligned}
$$

and hence (23) shows that

$$
\iota_{s}^{*}(z)=z+o(s), \quad \iota_{s}^{* \prime}(z)=1+o(s) \quad(s \downarrow 0)
$$

locally uniformly with respect to $z$. Thus

$$
\begin{aligned}
K\left(z, \bar{\zeta} ; \Omega_{t^{*}(s)}\right) & =K\left(\iota_{s}^{*}(z), \overline{\iota_{s}^{*}(\zeta)} ; \Omega_{s}^{*}\right) \sqrt{\iota_{s}^{* \prime}(z)} \overline{\sqrt{\iota_{s}^{* \prime}(\zeta)}} \\
& =K\left(z, \zeta ; \Omega_{s}^{*}\right)+o(s) \quad(s \downarrow 0)
\end{aligned}
$$

and

$$
\begin{aligned}
K(z, \bar{\zeta} ; \Omega-[0, s])= & \frac{1}{2 \pi} \int_{\partial \Omega_{s}^{*}} K\left(w, \bar{\zeta} ; \Omega-[0, s] \overline{K\left(w, \bar{z} ; \Omega_{s}^{*}\right) \mid} d w \mid\right. \\
= & \frac{1}{2 \pi} \int_{\partial(\Omega \cup, 1)} K\left(\iota_{s}^{*}(\xi), \bar{\zeta} ; \Omega-[0, s]\right) \overline{K\left(\iota_{s}^{*}(\xi), \bar{z} ; \Omega_{s}^{*}\right)}\left|\iota_{s}^{* \prime}(\xi)\right||d \xi| \\
& +\frac{1}{2 \pi} \int_{\partial\left[-1, s^{c}\right]} K\left(w, \zeta ; \Omega-[0, s] \overline{K\left(w, \bar{z} ; \Omega_{s}^{*}\right)}|d w|\right. \\
= & \frac{1}{2 \pi} \int_{\partial(\Omega-[0, s])} K(w, \bar{\zeta} ; \Omega-[0, s]) \overline{K\left(w, \bar{z} ; \Omega_{s}^{*}\right)}|d w|+o(s) \\
= & K\left(z, \bar{\zeta} ; \Omega_{s}^{*}\right)+o(s) \quad(s \downarrow 0),
\end{aligned}
$$

which shows that

$$
K\left(z, \bar{\zeta} ; \Omega_{t^{*}(s)}\right)=K(z, \bar{\zeta} ; \Omega-[0, s])+o(s) \quad(s \downarrow 0) .
$$

Let $s=s^{*}(t)$ denote the inverse function of $t^{*}(s)$. Then this equality is rewritten as

$$
\text { (26) } K\left(z, \bar{\zeta} ; \Omega_{t}\right)=K\left(z, \bar{\zeta} ; \Omega-\left[0, s^{*}(t)\right]\right)+o\left(s^{*}(t)\right) \quad(t \downarrow 0) .
$$

By Lemma 11, we have 


$$
\begin{aligned}
\frac{1+s^{*}(t)}{4} & =\gamma\left(\Lambda_{t}\right)=\frac{1+t}{4}+o(t) \quad(t \downarrow 0), \quad \text { i.e. , } \\
s^{*}(t) & =t+o(t) \quad(t \downarrow 0) .
\end{aligned}
$$

Using this equality, we have, in the same manner as above,

$$
K\left(z, \bar{\zeta} ; \Omega-\left[0, s^{*}(t)\right]\right)=K(z, \bar{\zeta} ; \Omega-[0, t])+o(t) \quad(t \downarrow 0) .
$$

Combined with (26), this equality yields (25). Consequently, the case I and (25) show that the right-derivative $\partial^{+} K\left(z, \bar{\zeta} ; \Omega_{0}\right) / \partial t$ exists and equals $\left.\frac{\partial K}{\partial t}(z, \bar{\zeta} ; \Omega-[0, t])\right|_{t=0}$.

Third step. We show that the right-derivative $\partial^{+} K\left(z, \bar{\zeta} ; \Omega_{t}\right) / \partial t$ exists in $(0,|\Gamma|]$.

Take the conformal mapping $h_{t}$ from $\Lambda_{t}^{c}$ onto $[-1,0]^{c}$ such that $h_{t}\left(w_{t}\right)=0 \quad(0<t \leq|\Gamma|)$. Then Lemma 9 shows that $h_{t}^{\prime}\left(w_{t}\right)$ exists and $h_{t}\left(\overline{\Gamma-\Gamma_{t}}\right) \in \mathscr{L}_{0}$. Since $h_{t}$ is conformal, we have $h_{t}^{\prime}\left(w_{t}\right)\left(d w_{t} / d t\right)>0$. Since

$$
K\left(z, \bar{\zeta} ; \Omega_{u}\right)=K\left(h_{t}(z), \overline{h_{t}(\zeta)} ; h_{t}\left(\Omega_{u}\right)\right) \sqrt{h_{t}^{\prime}(z)} \sqrt{\sqrt{h_{t}^{\prime}(\zeta)}} \quad(0<t \leq u),
$$

the second step shows that $\partial^{+} K\left(z, \bar{\zeta} ; \Omega_{t}\right) / \partial t$ exists and equals

$$
\text { (27) }\left.\frac{\partial K}{\partial s}\left(h_{t}(z), \overline{h_{t}(\zeta)} ; h_{t}\left(\Omega_{t}\right)-[0, s]\right)\right|_{s=0}\left|h_{t}^{\prime}\left(w_{t}\right)\right| \sqrt{h_{t}^{\prime}(z)} \sqrt{\sqrt{h_{t}^{\prime}(\zeta)}} .
$$

Fourth step. We show that $D K\left(w_{t}, z, \zeta ; \Omega_{t}\right)$ exists and is continuous in $(0,|\Gamma|]$.

Let $k_{t}$ be the conformal mapping in Lemma 10. Then we have, with $\hat{Q}_{t}=k_{t}\left(\Omega_{t}\right)$,

$$
\begin{array}{r}
D K\left(w_{u}, z, \zeta ; \Omega_{t}\right)=D K\left(k_{t}\left(w_{u}\right), k_{t}(z), k_{t}(\zeta) ; \hat{Q}_{t}\right)\left|k_{t}^{\prime}\left(w_{u}\right) k_{t}^{\prime}(z) k_{t}^{\prime}(\zeta)\right| \\
(0<t<u) .
\end{array}
$$

Since $J\left(\xi, \cdot ; \hat{Q}_{t}\right)(J=K, L)$ are analytic in a neighborhood of $r_{t}$ with respect to $\xi$, the right-hand side is rewritten as

$$
\begin{aligned}
& {\left[\left\{L\left(r_{t}, k_{t}(z) ; \hat{\Omega}_{t}\right)+\left(k_{t}\left(w_{u}\right)-r_{t}\right) \frac{\partial L}{\partial \xi}\left(r_{t}, k_{t}(z) ; \hat{\Omega}_{t}\right)+o(\sqrt{u-t})\right\}\right.} \\
& \quad \times \overline{\left\{L\left(r_{t}, k_{t}(\zeta) ; \hat{\Omega}_{t}\right)+\left(k_{t}\left(w_{u}\right)-r_{t}\right) \frac{\partial L}{\partial \xi}\left(r_{t}, k_{t}(\zeta) ; \hat{\Omega}_{t}\right)+o(\sqrt{u-t})\right\}} \\
& \quad-\left\{K\left(r_{t}, \overline{k_{t}(z)} ; \hat{Q}_{t}\right)+\left(k_{t}\left(w_{u}\right)-r_{t}\right) \frac{\partial K}{\partial \xi}\left(r_{t}, \overline{k_{t}(z)} ; \hat{Q}_{t}\right)+o(\sqrt{u-t})\right\}
\end{aligned}
$$




$$
\begin{aligned}
& \times\left\{K\left(r_{t}, \overline{k_{t}(\zeta)} ; \hat{\Omega}_{t}\right)+\left(k_{t}\left(w_{u}\right)-r_{t}\right) \frac{\partial K}{\partial \xi}\left(r_{t}, \overline{k_{t}(\zeta)} ; \hat{\Omega}_{t}\right)+o(\sqrt{u-t})\right\} \\
& \times\left|k_{t}^{\prime}\left(w_{u}\right) k_{t}^{\prime}(z) k_{t}^{\prime}(\zeta)\right| \quad(0<t<u)
\end{aligned}
$$

where $\frac{\partial J}{\partial \xi}\left(r_{t}, \cdot ; \hat{\Omega}_{t}\right)=\left.\frac{\partial J}{\partial \xi}\left(\xi, \cdot ; \hat{\Omega}_{t}\right)\right|_{\xi=r_{t}}(J=K, L)$. Recall the quantity $a_{t}$ in Lemma 10. Since

$$
L\left(r_{t}, k_{t}(z) ; \hat{\Omega}_{t}\right) \overline{L\left(r_{t}, k_{t}(\zeta) ; \hat{\Omega}_{t}\right)}=\overline{K\left(r_{t}, \overline{k_{t}(z)} ; \hat{\Omega}_{t}\right)} K\left(r_{t}, \overline{k_{t}(\zeta)} ; \hat{\Omega}_{t}\right),
$$

Lemma 10 shows that $D K\left(w_{t}, z, \zeta ; \Omega_{t}\right)$ exists and

$$
\text { (28) } \begin{aligned}
D K & \left(w_{t}, z, \zeta ; \Omega_{t}\right)=\left[L\left(r_{t}, k_{t}(z) ; \hat{\Omega}_{t}\right) \overline{\frac{\partial L}{\partial \xi}\left(r_{t}, k_{t}(\zeta) ; \hat{\Omega}_{t}\right)}\right. \\
& +\frac{\partial L}{\partial \xi}\left(r_{t}, k_{t}(z) ; \hat{\Omega}_{t}\right) \overline{L\left(r_{t}, k_{t}(\zeta) ; \hat{\Omega}_{t}\right)} \\
& -\overline{K\left(r_{t}, \overline{k_{t}(z)} ; \hat{Q}_{t}\right)} \frac{\partial K}{\partial \xi}\left(r_{t}, \overline{k_{t}(\zeta)} ; \hat{\Omega}_{t}\right) \\
& \left.-\overline{\frac{\partial K}{\partial \xi}\left(r_{t}, \overline{k_{t}(z)} ; \hat{\Omega}_{t}\right)} K\left(r_{t}, \overline{k_{t}(\zeta)} ; \hat{\Omega}_{t}\right)\right] a_{t}\left|k_{t}^{\prime}(z) k_{t}^{\prime}(\zeta)\right|
\end{aligned}
$$

Since $a_{t}$ is continuous in $(0,|\Gamma|], D K\left(w_{t}, z, \zeta ; \Omega_{t}\right)$ is continuous in $(0,|\Gamma|]$.

Fifth step. We show that $\partial^{+} K\left(z, \bar{\zeta} ; \Omega_{t}\right) / \partial t=D K\left(w_{t}, z, \zeta ; \Omega_{t}\right) / 4 \quad(0<t$ $\leq|\Gamma|)$.

Let $h_{t}$ be the same as in the third step. Then we have

(29) $D K\left(w_{u}, z, \zeta ; \Omega_{t}\right)$

$$
=D K\left(h_{t}\left(w_{u}\right), h_{t}(z), h_{t}(\zeta) ; h_{t}\left(\Omega_{t}\right)\right)\left|h_{t}^{\prime}\left(w_{u}\right)\right| \sqrt{h_{t}^{\prime}(z)} \sqrt{\sqrt{h_{t}^{\prime}(\zeta)}}(0<t<u) .
$$

In the same manner as in the fourth step,

$$
\lim _{u \downarrow t} D K\left(h_{t}\left(w_{u}\right), h_{t}(z), h_{t}(\zeta) ; h_{t}\left(\Omega_{t}\right)\right)=\lim _{s \downarrow 0} D K\left(s, h_{t}(z), h_{t}(\zeta) ; h_{t}\left(\Omega_{t}\right)\right) .
$$

The case I shows that this quantity is equal to

$$
\left.4 \frac{\partial K}{\partial s}\left(h_{t}(z), \overline{h_{t}(\zeta)} ; h_{t}\left(\Omega_{t}\right)-[0, s]\right)\right|_{s=0} .
$$

Lemma 9 shows that $\lim _{u \downarrow t} h_{t}^{\prime}\left(w_{u}\right)=h_{t}^{\prime}\left(w_{t}\right)$. Thus, letting $u$ tend to $t$ in (29), we see that $D K\left(w_{t}, z, \zeta ; \Omega_{t}\right) / 4$ exists and equals (27), which yields the required equality.

Final step. There remains to prove that the left-derivative 
$\partial^{-} K\left(z, \zeta ; \Omega_{t}\right) / \partial t$ exists in $\left(0,\left|I^{\top}\right|\right]$ and equals $\partial^{+} K\left(z, \xi ; \Omega_{t}\right) / \partial t$.

Fixing $0<t_{0}<|\Gamma|$, we form a function

$$
\rho^{*}(t)=K\left(z, \bar{\zeta} ; \Omega_{t}\right)-K\left(z, \bar{\zeta} ; \Omega_{t_{0}}\right)-\int_{t_{0}}^{t} \frac{\partial^{+}}{\partial s} K\left(z, \bar{\zeta} ; \Omega_{s}\right) d s
$$

on $\left[t_{0},|\Gamma|\right]$. Then $\rho^{*}\left(t_{0}\right)=0$ and the right-derivative is identically 0 , which yields that $\rho^{*}(t) \equiv 0$. Thus $\partial^{-} K\left(z, \bar{\zeta} ; \Omega_{t}\right) / \partial t$ exists and equals $\partial^{+} K\left(z, \bar{\zeta} ; \Omega_{t}\right) / \partial t$ in $\left(t_{0},|\Gamma|\right]$, and hence the derivative exists in $\left(t_{0},|\Gamma|\right]$. Since $0<t_{0}<|\Gamma|$ is arbitrary, $\partial K\left(z, \bar{\zeta} ; \Omega_{t}\right) / \partial t$ exists in $(0,|\Gamma|]$. This completes the proof of Theorem 8 in the case II.

\section{$\S 6$. Proof of Case III by the operator $\mathscr{H}$}

In this section, we prove Theorem 8 in the case III. This case corresponds to the variation by cutting a hole [28] and the proof is easier than that of the other cases. There are three methods of the proof. The first method is as follows: For a small number $t>0$, we define a competing function of form $K(z, \bar{\zeta} ; \Omega)+t_{\sigma}(z, \bar{\zeta}, t) D K\left(w_{0}, z, \zeta ; \Omega\right)$ so that the difference from $K\left(z, \zeta ; \Omega_{t}\right)$ is negligible. The second method is as follows: Taking a conformal mapping from $\Gamma_{t}^{c}$ outside a small disk, we use the reflection principle on the boundary of this disk in order to study $K\left(z, \bar{\zeta} ; \Omega_{t}\right)-K(z, \bar{\zeta} ; \Omega)$. In this note, we use the third method, which is an application of the singular integral operator $\mathscr{H}$ and completely different from the methods by conformal mappings. This method is not short, however, this is a powerful tool to study $\gamma(\cdot)$. Evidently, $D J\left(w_{0}, z, \zeta ; \Omega\right)$ $(J=K, L)$ exist. We shall see that (17) is still valid in this case. For $E \in \mathscr{A}, L^{2}(E)$ denotes the $L^{2}$-space of functions $\mu$ on $E$ with norm $\|\mu\|_{E}=$ $\left\{\int_{E}|\mu|^{2}|d z|\right\}^{1 / 2}$. The operator $\mathscr{H}_{E}$ from $L^{2}(E)$ to itself is defined by

$$
\begin{aligned}
\mathscr{H}_{E} \mu(z) & =\frac{1}{\pi} p \cdot v \cdot \int_{E} \frac{\mu}{\zeta-z}|d \zeta| \\
& =\frac{1}{\pi} \lim _{\varepsilon \downarrow 0} \int_{E,|\zeta-z|>\odot} \frac{1}{\zeta-z} \mu(\zeta)|d \zeta| \quad(z \in E) .
\end{aligned}
$$

We define $\overline{\mathscr{H}}_{E}$ by $\overline{\mathscr{H}}_{E} \mu=\overline{\mathscr{H}_{E} \bar{\mu}}$. The operator $\mathscr{H}_{E}$ is anti-symmetric, i.e., $\left(\mathscr{H}_{E} \mu, \nu\right)_{E}=-\left(\mu, \overline{\mathscr{H}}_{E} \nu\right)_{E}$, where $(\cdot, \cdot)_{E}$ is the inner product. The operator Id $-\mathscr{H}_{E} \overline{\mathscr{H}}_{E}$ is invertible and its inverse operator is denoted by $\mathscr{T}_{E}$, where Id is the identity operator. Using $\mathscr{T}_{E}$, we can express $K\left(z, \bar{\zeta} ; E^{c}\right)$, $L\left(z, \zeta ; E^{c}\right)$ in the following forms. 
Proposition 13.

(30) $K\left(z, \bar{\zeta} ; E^{c}\right)=\frac{1}{\pi} \int_{E} \frac{1}{w-z} \overline{\mathscr{T}}_{E}\left(\frac{1}{\cdot-\zeta}\right)(w)|d w|$,

(31) $L\left(z, \zeta ; E^{c}\right)=\frac{1}{z-\zeta}+\frac{1}{\pi} \int_{E} \frac{1}{w-z} \overline{\mathscr{H}}_{E} \mathscr{T}_{E}\left(\frac{1}{\cdot-\zeta}\right)(w)|d w|$.

To see this proposition, we begin by showing

(32) $\quad c\left(z ; E^{c}\right)=\frac{1}{\pi} \int_{E} \frac{1}{w-z} \overline{\mathscr{T}}_{E}\left(\frac{1}{\overline{-z}}\right)(w)|d w| \quad\left(z \in E^{c}-\{\infty\}\right)$.

The dual extremum problem yields that

$$
\begin{aligned}
c\left(z ; E^{c}\right) & =\inf \left\{\frac{1}{2 \pi} \int_{\partial E^{c}}\left|\frac{1}{w-z}+\psi(w)\right|^{2}|d w| ; \psi \in H^{2}\left(E^{c}\right), \psi(\infty)=0\right\} \\
& =\frac{1}{2 \pi} \int_{\partial E^{c}}\left|L\left(w, z ; E^{c}\right)\right|^{2}|d w| \\
& =\frac{1}{2 \pi} K\left(z, \bar{z} ; E^{c}\right) \quad[4, \text { Chap. VII }] .
\end{aligned}
$$

For $\psi \in H^{2}\left(E^{c}\right), \quad \psi(\infty)=0$, there exists $\mu \in L^{2}(E)$ such that $\psi(w)=$ $\frac{1}{\pi} \int_{E} \frac{\mu}{\zeta-w}|d \zeta|\left(w \in E^{c}\right)$. Then

$$
\psi(w)=\mathscr{H}_{E} \mu(w)+i \mu(w) \frac{|d w|}{d w} \text { a.e. on } \partial E^{c} .
$$

To each $w \in E-\left\{\right.$ endpoints of $\left.C_{j}(j=1, \cdots, n)\right\}$, there correspond two points $w^{ \pm} \in \partial E^{c}$ so that $d w^{+}=-d w^{-}$, where $\left\{C_{j}\right\}_{j=1}^{n}$ are closed analytic arcs such that $E=\bigcup_{j=1}^{n} C_{j}$. Since

$$
\begin{aligned}
& \frac{1}{2 \pi} \int_{\partial E^{c}}\left|\frac{1}{w-z}+\psi(w)\right|^{2}|d w| \\
& =\frac{1}{2 \pi} \int_{E}\left(\left|\frac{1}{w-z}+\mathscr{H}_{E} \mu(w)+i \mu(w) \frac{|d w|}{d w^{+}}\right|^{2}\right. \\
& \left.\quad+\left|\frac{1}{w-z}+\mathscr{H}_{E} \mu(w)+i \mu(w) \frac{|d w|}{d w^{-}}\right|^{2}\right)|d w| \\
& =\frac{1}{\pi}\left\{\mid \frac{1}{-z}+\mathscr{H}_{E} \mu\left\|_{E}^{2}+\right\| \mu \|_{E}^{2}\right\}
\end{aligned}
$$

we have

$$
c\left(z ; E^{c}\right)=\inf \left\{\frac{1}{\pi}\left(\left\|\frac{1}{\cdot-z}+\mathscr{H}_{E} \mu\right\|_{E}^{2}+\|\mu\|_{E}^{2}\right) ; \mu \in L^{2}(E)\right\} .
$$


There exists $\mu_{0} \in L^{2}(E)$ attaining $c\left(z ; E^{c}\right)$. Then a variational method shows that

$$
\left(\frac{1}{\cdot-z}+\mathscr{H}_{E} \mu_{0}, \mathscr{H}_{E} \mu\right)_{E}+\left(\mu_{0}, \mu\right)_{E}=0
$$

for all $\mu \in L^{2}(E)$, and hence

$$
\left(\mathrm{Id}-\overline{\mathscr{H}}_{E} \mathscr{H}_{E}\right) \mu_{0}=\overline{\mathscr{H}}_{E}\left\{\frac{1}{\cdot-z}\right\}, \quad \text { i.e., } \quad \mu_{0}=\overline{\mathscr{T}}_{E} \overline{\mathscr{H}}_{E}\left\{\frac{1}{\cdot-z}\right\} .
$$

Since Id $+\mathscr{H}_{E} \overline{\mathscr{T}}_{E} \overline{\mathscr{H}}_{E}=\mathscr{T}_{E}$, we have

$$
\begin{aligned}
c\left(z ; E^{c}\right) & =\frac{1}{\pi}\left\{\left\|\frac{1}{\cdot-z}+\mathscr{H}_{E} \mu_{0}\right\|_{E}^{2}+\left\|\mu_{0}\right\|_{E}^{2}\right\} \\
& =\frac{1}{\pi}\left(\frac{1}{\cdot-z}+\mathscr{H}_{E} \mu_{0}, \frac{1}{\cdot-z}\right)_{E}=\frac{1}{\pi} \int_{E} \frac{1}{w-z} \mathscr{T}_{E}\left\{\frac{1}{\cdot-z}\right\}(w)|d w| \\
& =\frac{1}{\pi} \int_{E} \frac{1}{w-z} \overline{\mathscr{T}}_{E}\left\{\frac{1}{\frac{1}{-z}}\right\}(w)|d w| .
\end{aligned}
$$

Thus (32) holds.

We now deduce (30) from (32). Let $K^{*}(z, \bar{\zeta}) / \pi$ be the kernel in the right-hand side of (30). For each $\zeta \in E^{c}, K^{*}(\cdot, \bar{\zeta})$ is analytic in $E^{c}$. Evidently, $K^{*}(\infty, \bar{\zeta})=0$ and $K^{*}(z, \bar{\zeta})=\overline{K^{*}(\zeta, \bar{z})}$. Equality (32) shows that

$$
\frac{1}{\pi} K\left(z, \bar{z} ; E^{c}\right)=c\left(z ; E^{c}\right)=\frac{1}{\pi} K^{*}(z, \bar{z}) \quad\left(z \in E^{c}\right) .
$$

Let

$$
K\left(z, \bar{\zeta} ; E^{c}\right)=\sum_{n=1}^{\infty} \sum_{m=1}^{\infty} a_{n, m} z^{-n \bar{\zeta}-\bar{\zeta}}, \quad K^{*}(z, \bar{\zeta})=\sum_{n=1}^{\infty} \sum_{m=1}^{\infty} b_{n, m} z^{-n \overline{\zeta^{-m}}}
$$

be the expansions at $\infty$. Then (33) yields that $a_{n, m}=b_{n, m}(m, n=1,2, \cdots)$ (cf. [30]). Thus $K\left(z, \bar{\zeta} ; E^{c}\right)=K^{*}(z, \bar{\zeta})$. We next prove (31). We have

$$
\begin{aligned}
L\left(z, \zeta ; E^{c}\right) & =\frac{1}{z-\zeta}+\frac{1}{2 \pi i} \int_{\partial E^{c}} \frac{1}{w-z}\left\{L\left(w, \zeta ; E^{c}\right)-\frac{1}{w-\zeta}\right\} d w \\
& =\frac{1}{z-\zeta}+\frac{1}{2 \pi} \int_{\partial E^{c}} \frac{1}{w-z} \overline{K\left(w, \bar{\zeta} ; E^{c}\right)}|d w|
\end{aligned}
$$

Equality (30) shows that

$$
K\left(w, \ddot{\zeta} ; E^{c}\right)=\mathscr{H}_{E} \overline{\mathscr{T}}_{E}\left(\frac{1}{\overline{-\zeta \zeta}}\right)(w)+i \overline{\mathscr{T}}_{E}\left(\frac{1}{\overline{-\zeta}}\right)(w) \frac{|d w|}{d w} \quad \text { a.e. on } \partial E^{c} \text {. }
$$


Replace $K\left(w, \bar{\zeta} ; E^{c}\right)$ in (34) by the right-hand side of this equality. Since $d w^{+}=-d w^{-}$a.e. on $E$, we obtain (31). This completes the proof of Propositon 13.

Using this proposition, we prove Theorem 8 in the case III. We divide the proof into three steps.

First step. We show that $\partial K\left(z, \bar{\zeta} ; \Omega_{0}\right) / \partial t$ exists and equals $D K\left(w_{0}, z, \zeta ; \Omega\right) / 4 . \quad\left(\partial K\left(z, \bar{\zeta} ; \Omega_{0}\right) / \partial t\right.$ means the right-derivative at $\left.t=0.\right)$

Let $\Omega \in \mathscr{G}$ and $\Gamma$ be a closed analytic arc in $\Omega$. Considering a suitable conformal mapping if necessary, we may assume that $\Omega \in \mathscr{F}$ and $w_{0}=0$. Let $E=\Omega^{c}, E_{t}=E \cup \Gamma_{t}(0<t \leq|\Gamma|)$. Given a small number $t>0$, we denote by $M$ the multiplier operator $\mu \rightarrow \chi_{E} \mu$ from $L^{2}\left(E_{t}\right)$ to itself, and denote by $N$ the multiplier operator $\mu \rightarrow \chi_{\Gamma_{t}} \mu$, where $\chi_{F}$ is the characteristic function of $F$. We write simply

$$
\mathscr{H}=\mathscr{H}_{E_{t}}, \quad \mathscr{T}=\mathscr{T}_{E_{t}}, \quad S=M \mathscr{T}_{E} M, \quad R=N \mathscr{T}_{T_{t}} N .
$$

Then (30) shows that

$$
K\left(z, \zeta ; \Omega_{t}\right)=\frac{1}{\pi}\left(\mathscr{T} \mu_{z}, \mu_{\zeta}\right), \quad K(z, \zeta ; \Omega)=\frac{1}{\pi}\left(S \mu_{z}, \mu_{b}\right),
$$

where $\mu_{\xi}(w)=1 /(w-\xi)(\xi=z, \zeta)$ and $(\cdot, \cdot)=(\cdot, \cdot)_{E t}$. To study $\mathscr{T}-S$, we rewrite the identity $(\operatorname{Id}-\mathscr{H} \mathscr{\mathscr { H }}) \mathscr{T}=\operatorname{Id}$ as

$$
\{\mathrm{Id}-\mathscr{H} M \overline{\mathscr{H}} M-\mathscr{H} N \overline{\mathscr{H}} N-\mathscr{H} M \overline{\mathscr{H}} N-\mathscr{H} N \overline{\mathscr{H}} M\} \mathscr{T}=\mathrm{Id} .
$$

Composing $S$ from the left-hand side, we have

$$
M \mathscr{T}=S+S \mathscr{H} N \overline{\mathscr{H}} N \mathscr{T}+S \mathscr{H} M \overline{\mathscr{H}} N \mathscr{T}+S \mathscr{H} N \overline{\mathscr{H}} M \mathscr{T} .
$$

Composing $R$ from the left-hand side, we have

$$
N \mathscr{T}=R+R \mathscr{H} M \overline{\mathscr{H}} M \mathscr{T}+R \mathscr{H} M \overline{\mathscr{H}} N \mathscr{T}+R \mathscr{H} N \overline{\mathscr{H}} M \mathscr{T} .
$$

Replace $N \mathscr{T}$ in (35) by the right-hand side of (36). Adding the resulting quantity and (36), we obtain

$$
\begin{aligned}
\mathscr{T}= & S+S \mathscr{H} N \overline{\mathscr{H}} R+S \mathscr{H} N \overline{\mathscr{H}} R \mathscr{H} M \overline{\mathscr{H}} M \mathscr{T}+S \mathscr{H} N \overline{\mathscr{H}} R \mathscr{H} M \overline{\mathscr{H}} N \mathscr{T} \\
& +S \mathscr{H} N \overline{\mathscr{H}} R \mathscr{H} N \overline{\mathscr{H}} M \mathscr{T}+S \mathscr{H} M \overline{\mathscr{H}} R+S \mathscr{H} M \overline{\mathscr{H}} R \mathscr{H} M \overline{\mathscr{H}} M \mathscr{T} \\
& +S \mathscr{H} M \overline{\mathscr{H}} R \mathscr{H} M \overline{\mathscr{H}} N \mathscr{T}+S \mathscr{H} M \overline{\mathscr{H}} R \mathscr{H} N \overline{\mathscr{H}} M \mathscr{T}+S \mathscr{H} N \overline{\mathscr{H}} M \mathscr{T} \\
& +R+R \mathscr{H} M \overline{\mathscr{H}} M \mathscr{T}+R \mathscr{H} M \overline{\mathscr{H}} N \mathscr{T}+R \mathscr{H} N \overline{\mathscr{H}} M \mathscr{T} \\
= & S+K_{1}+\cdots+K_{13}, \text { say. }
\end{aligned}
$$


In the estimates of $K_{j}(j=1, \cdots, 13)$, the following inequality plays an important role:

$$
\begin{aligned}
& \left|\left(\mathscr{H} M \mu, N_{\nu}\right)-\mathscr{H} M \mu(0)\left(1, N_{\nu}\right)\right| \\
& \quad \leq \frac{C}{\pi}\left|\Gamma_{\iota}\right| \int_{E}|\mu||d w| \int_{\Gamma_{t}}|\nu||d w| \quad\left(\mu, \nu \in L^{2}\left(E_{t}\right)\right),
\end{aligned}
$$

where $C=(\text { the distance between } E \text { and } \Gamma)^{-2}$. In fact,

$$
\left(\mathscr{H} M \mu, N_{\nu}\right)-\mathscr{H} M \mu(0)\left(1, N_{\nu}\right)=\frac{1}{\pi} \int_{\Gamma_{t}} \overline{\nu(z)}\left\{\int_{E} \frac{z}{(w-z) w} \mu(w)|d w|\right\}|d z|,
$$

which gives (37). Since $N \overline{\mathscr{H}} R$ is anti-symmetric, we have $(1, N \overline{\mathscr{H}} R 1)=0$, and hence

$$
\begin{aligned}
\left(K_{13} \mu_{z}, \mu_{\zeta}\right) & =-\left(\overline{\mathscr{H}} M \mathscr{T} \mu_{z}, N \overline{\mathscr{H}} R \mu_{\zeta}\right)=-\overline{\mathscr{H}} M \mathscr{T} \mu_{z}(0)\left(1, N \overline{\mathscr{H}} R \mu_{\zeta}\right)+o(t) \\
& =-\overline{\mathscr{H}} M \mathscr{T} \mu_{z}(0) \overline{\mu_{\zeta}}(0)(1, N \overline{\mathscr{H}} R 1)+o(t)=o(t) .
\end{aligned}
$$

Since

$$
(1, R 1)=\pi \gamma\left(\Gamma_{t}\right)=\frac{\pi}{4} t+o(t) \quad[18], \quad \mathscr{H} M \overline{\mathscr{H}} N \mathscr{T} \mu_{z}(0)=o(1)
$$

we have

$$
\begin{aligned}
\left(K_{12} \mu_{z}, \mu_{\xi}\right) & =\left(\mathscr{H} M \overline{\mathscr{H}} N \mathscr{T} \mu_{z}, R \mu_{\zeta}\right)=\mathscr{H} M \overline{\mathscr{H}} N \mathscr{T} \mu_{z}(0)\left(1, R \mu_{\zeta}\right)+o(t) \\
& =\mathscr{H} M \overline{\mathscr{H}} N \mathscr{T} \mu_{z}(0) \overline{\mu_{\zeta}(0)}(1, R 1)+o(t)=o(t) .
\end{aligned}
$$

Since $\mathscr{H} M \overline{\mathscr{H}} M \mathscr{T} \mu_{z}(0)=\mathscr{H} M \overline{\mathscr{H}} S \mu_{z}(0)+o(1)$, we have

$$
\begin{aligned}
\left(K_{11} \mu_{z}, \mu_{\zeta}\right) & =\left(\mathscr{H} M \overline{\mathscr{H}} M \mathscr{T} \mu_{z}, R \mu_{\zeta}\right)=\mathscr{H} M \overline{\mathscr{H}} M \mathscr{T} \mu_{z}(0) \overline{\mu_{\zeta}(0)}(1, R 1)+o(t) \\
& =\frac{\pi}{4} t \mathscr{H} M \overline{\mathscr{H}} S \mu_{z}(0) \overline{\mu_{\zeta}(0)}+o(t) .
\end{aligned}
$$

In the same manner, we have

$$
\begin{aligned}
\left(K_{10} \mu_{z}, \mu_{\zeta}\right) & =\mu_{z}(0) \overline{\mu_{\zeta}(0)}(R 1,1)+o(t)=\frac{\pi}{4} t \mu_{z}(0) \overline{\mu_{\zeta}(0)}+o(t), \\
\left(K_{9} \mu_{z}, \mu_{\zeta}\right) & =-\left(\overline{\mathscr{H}} M \mathscr{T} \mu_{z}, N \overline{\mathscr{H}} S \mu_{\zeta}\right)=-\overline{\mathscr{H}} M \mathscr{T} \mu_{z}(0) \mathscr{H} \overline{S \mu_{\zeta}}(0)(1, N 1)+o(t) \\
& =-t \overline{\mathscr{H}} S \mu_{z}(0) \mathscr{H} \overline{S \mu_{\zeta}}(0)+o(t), \\
\left(K_{6} \mu_{z}, \mu_{\zeta}\right) & =\frac{\pi}{4} t \mathscr{H} M \overline{\mathscr{H}} S \mu_{z}(0) \overline{\mathscr{H}} M \overline{\mathscr{H} S \mu_{\zeta}}(0)+o(t), \\
\left(K_{5} \mu_{z}, \mu_{\zeta}\right) & =\frac{\pi}{4} t \mu_{z}(0) \overline{\mathscr{H}} M \mathscr{H} \overline{S \mu_{\zeta}}(0)+o(t),
\end{aligned}
$$




$$
\begin{aligned}
\left(K_{4} \mu_{z}, \mu_{\zeta}\right) & =-\overline{\mathscr{H}} M \mathscr{T} \mu_{z}(0) \mathscr{H} \overline{S \mu_{\zeta}}(0)(N \overline{\mathscr{H}} R \mathscr{H} N 1,1)+o(t) \\
& =-\left(\frac{\pi}{4}-1\right) t \overline{\mathscr{H}} S \mu_{z}(0) \mathscr{H} \bar{S} \mu_{\zeta}(0)+o(t), \\
\left(K_{j} \mu_{z}, \mu_{\xi}\right) & =o(t) \quad(j=1,2,3,7,8) .
\end{aligned}
$$

Proposition 13 shows that

$$
\overline{\mathscr{H}} S \mu_{\xi}(0)=\overline{K(0, \bar{\xi} ; \Omega)}, \quad \mu_{\xi}(0)+\mathscr{H} M \overline{\mathscr{H}} S \mu_{\xi}(0)=L(0, \xi ; \Omega) \quad(\xi=z, \zeta) .
$$

Thus

$$
\begin{aligned}
& \pi\left\{K\left(z, \bar{\zeta} ; \Omega_{t}\right)-K(z, \bar{\zeta} ; \Omega)\right\} \\
& =\left(\mathscr{T} \mu_{z}, \mu_{\zeta}\right)-\left(S \mu_{z}, \mu_{\zeta}\right)=\sum_{j=1}^{13}\left(K_{j} \mu_{z}, \mu_{\zeta}\right) \\
& =\frac{\pi}{4} t\left\{\mu_{z}(0) \overline{\mu_{\xi}(0)}+\mathscr{H} M \overline{\mathscr{H}} S \mu_{z}(0) \overline{\mu_{\zeta}(0)}+\mu_{z}(0) \overline{\mathscr{H}} M \mathscr{\mathscr { H }} \overline{S \mu_{\zeta}}(0)\right. \\
& \left.+\mathscr{H} M \overline{\mathscr{H}} S \mu_{z}(0) \overline{\mathscr{H}} M \mathscr{H} \overline{S \mu_{\xi}}(0)-\overline{\mathscr{H}} S \mu_{z}(0) \mathscr{H} \overline{S \mu_{\xi}}(\mathrm{C})\right\}+o(t) \\
& =\frac{\pi}{4} t\{(L(0, z ; \Omega) \overline{L(0, \zeta ; \Omega)}-\overline{K(0, \bar{z} ; \Omega)} K(0, \bar{\zeta} ; \Omega)+o(t) \\
& =\frac{\pi}{4} t D K(0, z, \zeta ; \Omega)+o(t),
\end{aligned}
$$

which shows that $\partial K\left(z, \bar{\zeta} ; \Omega_{0}\right) / \partial t$ exists and equals $D K(0, z, \zeta ; \Omega) / 4$.

Second step. We show that $\partial L\left(z, \zeta ; \Omega_{0}\right) / \partial t$ exists and equals $D L(0, z, \zeta ; \Omega) / 4$.

We may assume that $z \neq \zeta$. Equality (31) shows that

$$
\begin{aligned}
& L\left(z, \zeta ; \Omega_{t}\right)=\frac{1}{z-\zeta}+\frac{1}{\pi}\left(\overline{\mathscr{H}} \mathscr{T} \mu_{\zeta}, \bar{\mu}_{z}\right), \\
& L(z, \zeta ; \Omega)=\frac{1}{z-\zeta}+\frac{1}{\pi}\left(M \overline{\mathscr{H}} S \mu_{\zeta}, \bar{\mu}_{z}\right) .
\end{aligned}
$$

To study $\overline{\mathscr{H}} \mathscr{T}-M \overline{\mathscr{H}} S$, we rewrite the identity $(\mathrm{Id}-\overline{\mathscr{H}} \mathscr{H}) \overline{\mathscr{H}} \mathscr{T}=\overline{\mathscr{H}}$ as

$$
\{\mathrm{Id}-\overline{\mathscr{H}} M \mathscr{H} M-\overline{\mathscr{H}} N \mathscr{H} N-\overline{\mathscr{H}} M \mathscr{H} N-\overline{\mathscr{H}} N \mathscr{H} M\} \overline{\mathscr{H}} \mathscr{T}=\overline{\mathscr{H}}
$$

Composing $\bar{S}$ from the left-hand side, we have

$$
M \overline{\mathscr{H}} \mathscr{T}=\overline{S \mathscr{H}}+\overline{S \mathscr{H}} N \mathscr{H} N \overline{\mathscr{H}} \mathscr{T}+\overline{S \mathscr{H}} M \mathscr{H} N \overline{\mathscr{H}} \mathscr{T}+\overline{S \mathscr{H}} N \mathscr{H} M \overline{\mathscr{H}} \mathscr{T} .
$$

Composing $\bar{R}$ from the left-hand side, we have

$$
N \overline{\mathscr{H}} \mathscr{T}=\overline{R \mathscr{H}}+\overline{R \mathscr{H}} M \mathscr{H} M \overline{\mathscr{H}} \mathscr{T}+\overline{R \mathscr{H}} M \mathscr{H} N \overline{\mathscr{H}} \mathscr{T}+\overline{R \mathscr{H}} N \mathscr{H} M \overline{\mathscr{H}} \mathscr{T} .
$$


Using these identities, we obtain, in the same manner as above,

$$
\begin{aligned}
& \overline{\mathscr{H}} \mathscr{T}=\overline{S \mathscr{H}}+\overline{S \mathscr{H}} N \mathscr{H} \overline{R \mathscr{H}}+\overline{S \mathscr{H}} N \mathscr{H} \overline{R \mathscr{H}} M \mathscr{H} M \overline{\mathscr{H}} \mathscr{T} \\
& +\overline{S \mathscr{H}} N \mathscr{H} \overline{R \mathscr{H}} M \mathscr{H} N \overline{\mathscr{H}} \mathscr{T}+\overline{S \mathscr{H}} N \mathscr{H} \overline{R \mathscr{H}} N \mathscr{H} M \overline{\mathscr{H}} \mathscr{T} \\
& +\overline{S \mathscr{H}} M \mathscr{H} \overline{R \mathscr{H}}+\overline{S \mathscr{H}} M \mathscr{\mathscr { H }} \overline{R \mathscr{H}} M \mathscr{H} M \overline{\mathscr{H}} \mathscr{T} \\
& +\overline{S \mathscr{H}} M \mathscr{H} \overline{R \mathscr{H}} M \mathscr{H} N \overline{\mathscr{H}} \mathscr{T}+\overline{S \mathscr{H}} M \mathscr{H} \overline{R \mathscr{H}} N \mathscr{H} M \overline{\mathscr{H}} \mathscr{T} \\
& +\overline{S \mathscr{H}} N \mathscr{H} M \overline{\mathscr{H}} \mathscr{T}+\overline{R \mathscr{H}}+\overline{R \mathscr{H}} M \mathscr{H} M \overline{\mathscr{H}} \mathscr{T}+\overline{R \mathscr{H}} M \mathscr{H} N \overline{\mathscr{H}} \mathscr{T} \\
& +\overline{R \mathscr{H}} N \mathscr{H} M \overline{\mathscr{H}} \mathscr{T}=\overline{S \mathscr{H}}+L_{1}+\cdots+L_{13} \text {, say. }
\end{aligned}
$$

In the same manner as above,

$$
\begin{aligned}
\left(\overline{S \mathscr{H}} \mu_{\zeta}, \mu_{z}\right) & =-\left(\mu_{\zeta}, M \mathscr{\mathscr { H }} \bar{S} \mu_{z}\right)-t \mu_{\zeta}(0) \overline{\mathscr{H}} S \mu_{z}(0)+o(t) \\
& =\left(M \overline{\mathscr{H}} S \mu_{\zeta}, \bar{\mu}_{z}\right)-t \mu_{\zeta}(0) \overline{\mathscr{H}} S \mu_{z}(0)+o(t), \\
\left(L_{1} \mu_{\zeta}, \bar{\mu}_{z}\right) & =-\left(\frac{\pi}{4}-1\right) t \mu_{\zeta}(0) \overline{\mathscr{H}} S \mu_{z}(0)+o(t), \\
\left(L_{4} \mu_{\zeta}, \bar{\mu}_{z}\right) & =-\left(\frac{\pi}{4}-1\right) t \mathscr{H} M \overline{\mathscr{H}} S \mu_{\zeta}(0) \overline{\mathscr{H}} S \mu_{z}(0)+o(t), \\
\left(L_{5} \mu_{\zeta}, \bar{\mu}_{z}\right) & =\frac{\pi}{4} t \overline{\mathscr{H}} M \mu_{\zeta}(0) \mathscr{H} M \overline{\mathscr{H}} S \mu_{z}(0)+o(t), \\
\left(L_{6} \mu_{\zeta}, \bar{\mu}_{z}\right) & =\frac{\pi}{4} t \overline{\mathscr{H}} M \mathscr{H} M \overline{\mathscr{H}} S \mu_{\zeta}(0) \mathscr{H} M \overline{\mathscr{H}} S \mu_{z}(0)+o(t), \\
\left(L_{9} \mu_{\zeta}, \bar{\mu}_{z}\right) & =-t \mathscr{H} M \overline{\mathscr{H}} S \mu_{\zeta}(0) \overline{\mathscr{H}} S \mu_{z}(0)+o(t), \\
\left(L_{10} \mu_{\zeta}, \bar{\mu}_{z}\right) & =\frac{\pi}{4} t \overline{\mathscr{H}} M \mu_{\zeta}(0) \mu_{z}(0)+o(t), \\
\left(L_{11} \mu_{\zeta}, \bar{\mu}_{z}\right) & =\frac{\pi}{4} t \overline{\mathscr{H}} M \mathscr{H} M \mathscr{\mathscr { H }} S \mu_{\zeta}(0) \mu_{z}(0)+o(t), \\
\left(L_{j} \mu_{\zeta}, \bar{\mu}_{z}\right) & =o(t) \quad(j=2,3,7,8,12,13) .
\end{aligned}
$$

Thus, by (38), we obtain

$$
\begin{aligned}
& \pi\left\{L\left(z, \zeta ; \Omega_{t}\right)-L(z, \zeta ; \Omega)\right\} \\
&=\left(\overline{\mathscr{H}} \mathscr{T} \mu_{\zeta}, \bar{\mu}_{z}\right)-\left(M \overline{\mathscr{H}} S \mu_{\zeta}, \bar{\mu}_{z}\right)=-t \mu_{\zeta}(0) \overline{\mathscr{H}} S \mu_{z}(0)+\sum_{j=1}^{13}\left(L_{j} \mu_{\zeta}, \bar{\mu}_{z}\right) \\
&=\frac{\pi}{4} t\left[\left\{\mu_{z}(0)+\mathscr{H} M \overline{\mathscr{H}} S \mu_{z}(0)\right\}\left\{\overline{\mathscr{H}} M \mu_{\zeta}(0)+\overline{\mathscr{H}} M \mathscr{H} M \overline{\mathscr{H}} S \mu_{\zeta}(0)\right\}\right. \\
&\left.-\overline{\mathscr{H}} S \mu_{z}(0)\left\{\mu_{\zeta}(0)+\mathscr{H} M \overline{\mathscr{H}} S \mu_{\zeta}(0)\right\}\right]+o(t) \\
&= \frac{\pi}{4} t\{L(0, z ; \Omega) \overline{K(0, \bar{\zeta} ; \Omega)}-\overline{K(0, \bar{z} ; \Omega)} L(0, \zeta ; \Omega)\}+o(t) \\
&= \frac{\pi}{4} t D L(0, z, \zeta ; \Omega)+o(t),
\end{aligned}
$$


which shows that $\partial L\left(z, \zeta ; \Omega_{0}\right) / \partial t$ exists and equals $D L(0, z, \zeta ; \Omega) / 4$.

Final step. We show that $D J\left(w_{t}, z, \zeta ; \Omega_{t}\right) / \partial t(J=K, L)$ are right-continuous at $t=0$.

We may assume that $w_{0}=0$. Here is a lemma necessary for the proof.

LEMma 14. Let $k_{t}, r_{t}(0<t \leq|\Gamma|)$ be the same as in Lemma 10. Then we have, with $\hat{Q}_{t}=k_{t}\left(\Omega_{t}\right)$,

$$
\begin{aligned}
& \lim _{t \downarrow 0} K\left(r_{t}, \overline{k_{t}(z)} ; \hat{Q}_{t}\right)=K(0, \bar{z} ; \Omega)-\overline{L(0, z ; \Omega)}, \\
& \lim _{t \downarrow 0} L\left(r_{t}, k_{t}(z) ; \hat{Q}_{t}\right)=L(0, z ; \Omega)-\overline{K(0, \bar{z} ; \Omega),} \\
& \lim _{t \downarrow 0} r_{t} \frac{\partial K}{\partial \xi}\left(r_{t}, \overline{k_{t}(z)} ; \hat{Q}_{t}\right)=\overline{L(0, z ; \Omega)}, \\
& \lim _{t \downarrow 0} r_{t} \frac{\partial L}{\partial \xi}\left(r_{t}, k_{t}(z) ; \hat{Q}_{t}\right)=\overline{K(0, \bar{z} ; \Omega),}
\end{aligned}
$$

where $\frac{\partial J}{\partial \xi}\left(r_{\iota}, \cdot ; \hat{Q}_{t}\right)=\left.\frac{\partial J}{\partial \xi}\left(\xi, \cdot ; \hat{Q}_{t}\right)\right|_{\xi=r_{t}} \quad(J=K, L)$.

Choose $\varepsilon_{0}>0$ so that $\overline{D\left(0, \varepsilon_{0}\right)} \subset \Omega$ and $k_{t}(z) \in \Omega-\overline{D\left(0, \varepsilon_{0}\right)}(0<t \leq|\Gamma|)$. Then $K\left(\xi, \overline{k_{t}(z)} ; \hat{Q}_{t}\right), L\left(\xi, k_{t}(z) ; \hat{Q}_{t}\right)$ uniformly converge to $K(\xi, \bar{z} ; \Omega)$, $L(\xi, z ; \Omega)$, respectively, on $\xi \in S\left(0, \varepsilon_{0}\right)$, when $t \downarrow 0$. Form

$$
\mathscr{K}_{t}(\xi)= \begin{cases}K\left(\xi, \overline{k_{t}(z)} ; \hat{Q}_{t}\right) & \left(\xi \in D\left(0, \varepsilon_{0}\right)-D\left(0, r_{t}\right)\right) \\ -\overline{L\left(r_{t}^{2} / \bar{\xi}, k_{t}(z) ; \hat{Q}_{t}\right)} r_{t} / \xi & \left(\xi \in D\left(0, r_{t}\right)-\overline{D\left(0, r_{t}^{2} / \varepsilon_{0}\right)}\right) .\end{cases}
$$

Since

$$
K\left(\xi, \overline{k_{t}(z)} ; \hat{Q}_{t}\right) \xi=-\overline{L\left(\xi, k_{t}(z) ; \hat{Q}_{t}\right)} r_{t} \quad\left(\xi \in S\left(0, r_{t}\right)\right),
$$

$\mathscr{K}_{t}(\xi)$ is analytic in $D\left(0, \varepsilon_{0}\right)-\overline{D\left(0, r_{t}^{2} / \varepsilon_{0}\right)}$. This shows that

$$
\begin{aligned}
& K\left(r_{t}, \overline{k_{t}(z)} ; \hat{\Omega}_{t}\right)=\frac{1}{2 \pi i} \int_{\partial\left\{D\left(0, \varepsilon_{0}\right)-D\left(0, r_{t}^{2} / \varepsilon_{0}\right)\right\}} \frac{1}{\xi-r_{t}} \mathscr{K}_{t}(\xi) d \xi \\
&=\frac{1}{2 \pi} \int_{0}^{2 \pi} \frac{1}{\varepsilon_{0} e^{i \psi}-r_{t}} K\left(\varepsilon_{0} e^{i \psi}, \overline{k_{t}(z)} ; \hat{\Omega}_{t}\right) \varepsilon_{0} e^{i \psi} d \psi \\
& \quad+\frac{1}{2 \pi} \int_{0}^{2 \pi} \frac{1}{\left(r_{t}^{2} / \varepsilon_{0}\right) e^{i \psi}-r_{t}} \overline{L\left(\varepsilon_{0} e^{i \psi}, k_{t}(z) ; \hat{Q}_{t}\right)} r_{t} d \psi \\
&= \frac{1}{2 \pi} \int_{0}^{2 \pi} K\left(\varepsilon_{0} e^{i \psi}, \bar{z} ; \Omega\right) d \psi-\frac{1}{2 \pi} \int_{0}^{2 \pi} L\left(\varepsilon_{0} e^{i \psi}, z ; \Omega\right) d \psi+o(1) \\
&= K(0, \bar{z} ; \Omega)-\overline{L(0, z ; \Omega)}+o(1) \quad(t \downarrow 0),
\end{aligned}
$$


which gives (39). Since

$$
\begin{aligned}
& r_{t} \frac{\partial K}{\partial \xi}\left(r_{t}, \overline{k_{t}(z)} ; \hat{Q}_{t}\right)=\frac{1}{2 \pi i} \int_{\partial\left\{D\left(0, \varepsilon_{0}\right)-D\left(0, r_{t}^{2} / \varepsilon_{0}\right)\right\}} \frac{r_{t}}{\left(\xi-r_{t}\right)^{2}} \mathscr{K}_{t}(\xi) d \xi \\
& =\frac{1}{2 \pi} \int_{0}^{2 \pi} \frac{r_{t}}{\left(\varepsilon_{0} e^{i \psi}-r_{t}\right)^{2}} K\left(\varepsilon_{0} e^{i \psi}, \overline{k_{t}(z)} ; \hat{\Omega}_{t}\right) \varepsilon_{0} e^{i \psi} d \psi \\
& +\frac{1}{2 \pi} \int_{0}^{2 \pi} \frac{r_{t}}{\left\{\left(r_{t}^{2} / \varepsilon_{0}\right) e^{i \psi}-r_{t}\right\}^{2}} \overline{L\left(\varepsilon_{0} e^{i \psi}, k_{t}(z) ; \hat{Q}_{t}\right)} r_{t} d \psi \\
& =\overline{\frac{1}{2 \pi} \int_{0}^{2 \pi} L\left(\varepsilon_{0} e^{i \psi}, z ; \Omega\right) d \psi}+o(1)=\overline{L(0, z ; \Omega)}+o(1) \text {, }
\end{aligned}
$$

which gives (41). In the same manner, we obtain (40) and (42). This completes the proof of Lemma 14 .

Recall (28) and $\lim _{t \downarrow 0} a_{t} / r_{t}=1 / 2$ in Lemma 10. Lemma 14 shows that

$$
\begin{aligned}
& \lim _{\iota \downarrow 0} D K\left(w_{t}, z, \zeta ; \Omega_{t}\right)=\frac{1}{2}[\{L(0, z ; \Omega)-\overline{K(0, \bar{z} ; \Omega)}\} K(0, \bar{\zeta} ; \Omega) \\
& +\overline{K(0, \bar{z} ; \Omega)}\{\overline{L(0, \zeta ; \Omega)}-K(0, \bar{\zeta} ; \Omega)\}-\{\overline{K(0, \bar{z} ; \Omega)}-L(0, z ; \Omega)\} \overline{L(0, \zeta ; \Omega)} \\
& -L(0, z ; \Omega)\{K(0, \bar{\zeta} ; \Omega)-\overline{L(0, \zeta ; \Omega)}\}] \\
& =L(0, z ; \Omega) \overline{L(0, \zeta ; \Omega)}-\overline{K(0, \bar{z} ; \Omega)} K(0, \bar{\zeta} ; \Omega)=D K(0, z, \zeta ; \Omega) \text {. }
\end{aligned}
$$

Thus $D K\left(w_{t}, z, \zeta ; \Omega_{t}\right)$ is continuous at $t=0$, i.e., $\partial K\left(z, \bar{\zeta} ; \Omega_{t}\right) / \partial t$ is continuous at $t=0$. In the same manner as in the proof of (28), we have

$$
\begin{aligned}
& D L\left(w_{t}, z, \zeta ; \Omega_{l}\right)=\left[L\left(r_{t}, k_{t}(z) ; \hat{Q}_{t}\right) \frac{\overline{\partial K}\left(r_{t}, \overline{k_{t}(\zeta)} ; \hat{Q}_{t}\right)}{\partial \xi}\right. \\
& +\frac{\partial L}{\partial \xi}\left(r_{t}, k_{t}(z) ; \hat{Q}_{t}\right) \overline{K\left(r_{t}, \overline{k_{t}(\zeta)} ; \hat{Q}_{t}\right)}-\overline{K\left(r_{t}, \overline{k_{t}(z)} ; \hat{Q}_{t}\right)} \frac{\partial L}{\partial \xi}\left(r_{t}, k_{t}(\zeta) ; \hat{Q}_{t}\right) \\
& \left.-\frac{\partial K}{\partial \xi}\left(r_{t}, \overline{k_{t}(z)} ; \hat{Q}_{t}\right) L\left(r_{t}, k_{t}(\zeta) ; \hat{Q}_{t}\right)\right] a_{t} \sqrt{k_{t}^{\prime}(z)} \sqrt{k_{t}^{\prime}(\zeta)},
\end{aligned}
$$

and hence, using Lemma 14 , we see that $D L\left(w_{t}, z, \zeta ; \Omega_{t}\right)$ is continuous at $t=0$, i.e., $\partial L\left(z, \zeta ; \Omega_{t}\right) / \partial t$ is continuous at $t=0$. This completes the proof of Theorem 8 in the case III.

\section{§ 7. Proof of Case IV}

In this section, we prove Theorem 8 in the case IV. We work only with $K$; the argument for $L$ is analogous. Let $\Omega \in \mathscr{G}$ and let $\Gamma$ be a closed analytic arc with the arc-length representation $w_{t}(0 \leq t \leq|\Gamma|)$ such that $\bar{Q} \supset \Gamma$ and $\partial \Omega \cap \Gamma=\left\{w_{0}\right\}$. Without loss of generality, we may assume 
that $w_{0}=0$. We show that $\partial K\left(z, \bar{\zeta} ; \Omega_{0}\right) / \partial t$ exists and $\partial K\left(z, \bar{\zeta} ; \Omega_{t}\right) / \partial t$ is continuous at $t=0$. There exists a component $\Lambda \in \mathscr{A}$ of $\Omega^{c}$ with an endpoint 0 . Let $h$ be the conformal mapping from $\Lambda^{c}$ onto $[-1,0]^{c}$ such that $h(\infty)=\infty$ and $h(0)=0$. Then Lemma 9 shows that $h(\Gamma) \in \mathscr{L}_{\theta}$ for some $-\pi<\theta \leq \pi$. If $\theta=0$, then the case II yields the required properties. Thus we may assume that $\theta \neq 0$. Let $w_{s}^{*}(0 \leq s \leq|h(\Gamma)|)$ be the arc-length representation of $h(\Gamma)$ such that $w_{0}^{*}=0$, and let $s=\hat{s}(t)$ be the number defined by $w_{s}^{*}=h\left(w_{t}\right)$. If the required properties are established for $h(\Omega)$ and $h(\Gamma)$, then

$$
\begin{aligned}
& \frac{\partial K}{\partial t}\left(z, \bar{\zeta} ; \Omega_{t}\right)=\frac{\partial K}{\partial s}\left(h(z), \overline{h(\zeta)} ; h(\Omega)-\left\{w_{x}^{*} ; 0 \leq x \leq s\right\}\right) \\
& \times h^{\prime}\left(w_{t}\right) \frac{d w_{t}}{d t}\left(\frac{d w_{s}^{*}}{d s}\right)^{-1} \sqrt{h^{\prime}(z)} \overline{\sqrt{h^{\prime}(\zeta)}} \quad(0 \leq t \leq|\Gamma|) .
\end{aligned}
$$

Lemma 9 shows that $h^{\prime}\left(w_{t}\right)\left(d w_{t} / d t\right)\left(d w_{s}^{*} / d s\right)^{-1}$ is right-continuous at $t=0$, which yields that $\partial K\left(z, \bar{\zeta} ; \Omega_{0}\right) / \partial t$ exists and $\partial K\left(z, \bar{\zeta} ; \Omega_{t}\right) / \partial t$ is right-continuous at $t=0$. Thus, from the beginning, we may assume that $\Lambda=[-1,0]$ and $\Gamma \in \mathscr{L}_{\theta}, \theta \neq 0$. We put $\Lambda_{t}=[-1,0] \cup \Gamma_{t}(0 \leq t \leq|\Gamma|)$. Here are some lemmas necessary for the proof.

Lemma 15. For an analytic function $P$ in $\mathbf{D}$, we put

$$
\begin{aligned}
& U_{s}=\left\{\tau_{s}(y) ; 0 \leq y \leq 1\right\}, \quad \tau_{s}(y)=i e^{i \theta / 2} y+\sqrt{s} y^{2} P(\sqrt{s} y), \\
& V_{s}=U_{s} \cup \bar{U}_{s} \quad(0 \leq s<1) .
\end{aligned}
$$

Then

$$
\lim _{s ! 0} \gamma\left(V_{s}\right)=\gamma\left(V_{0}\right)
$$

The sequence $\left\{V_{s}\right\}_{0<s<1}$ converges to $V_{0}$ as $s \downarrow 0$, however, (43) is not obvious. Take, for example,

$$
E_{n}=\bigcup_{k=0}^{n-1}\left\{\left[\frac{2 k}{2 n}, \frac{2 k+1}{2 n}\right] \cup\left(\frac{1+i}{2 n}+\left[\frac{2 k}{2 n}, \frac{2 k+1}{2 n}\right]\right)\right\} \quad(n=1,2, \cdots) .
$$

This sequence converges to $[0,1]$ but $\gamma([0,1])>\lim _{n \rightarrow \infty} \gamma\left(E_{n}\right)$. (See [18]). In the proof of (43), the connectivity of $V_{s}$ is important. Take the Green's function $G_{s}(z, \infty)$ of $V_{s}^{c}$ with pole at $\infty(0 \leq s<1)$. Then we see that $\lim _{s \downarrow 0} G_{s}(z, \infty)=G_{0}(z, \infty)$. The Ahlfors function $f\left(z ; V_{s}^{c}\right)$ of $V_{s}$ is expressed as $-\exp \left\{-G_{s}(z, \infty)-i G_{s}^{*}(z, \infty)\right\}$, where $G_{s}^{*}(z, \infty)$ is the conjugate multivalued harmonic function of $G_{s}(z, \infty)$ such that $G_{s}^{*}(\infty, \infty)=0(\bmod 2 \pi)$. Thus $\lim _{s \downarrow 0} f\left(z ; V_{s}^{c}\right)=f\left(z ; V_{0}^{c}\right)$, which gives (43). 
Lemma 16. Let $V_{s}, \tau_{s}(0 \leq s<1)$ be the same as in Lemma 15. Let

$$
\alpha_{s}=\lim _{y ! 1}\left\{q\left(\tau_{s}(y) ; V_{s}^{c}\right)-q\left(\tau_{s}(1) ; V_{s}^{c}\right)\right\} / \sqrt{y-1} \quad(0 \leq s<1),
$$

where $q$ is the function defined in the beginning of $\S 4$. Then $\lim _{s \downarrow 0} \alpha_{s}=\alpha_{0}$.

To prove this lemma, we write simply $\hat{q}_{s}(z)=q\left(z ; V_{s}^{c}\right)(0 \leq s<1)$. Lemma 15 shows that $\left\{\hat{q}_{s}\right\}_{0<s<1}$ locally uniformly converges to $\hat{q}_{0}$ as $s \downarrow 0$, and hence $\hat{q}_{s}(S(0,2)) \subset \overline{D(0, M)}-D(0,1 / M)(0<s<1)$ for some $M>1$. Since $\hat{q}_{s}\left(V_{s}\right)=S(0,1)$ and $\hat{q}_{s}$ does not take 0 in $V_{s}^{c}$, we have $\hat{q}_{s}\left(\overline{D(0,2)}-V_{s}\right)$ $\subset \overline{D(0, M)}-D(0,1 / M)$. There exists $\varepsilon_{0}>0$ such that the ranges of a semi-disk $D\left(0, \varepsilon_{0}\right) \cap\{\operatorname{Re} z>0\}$ by analytic functions $\tau_{s}\left(1+z^{2}\right)(0<s<1)$ are contained in $\overline{D(0,2)}$. Form $l_{s}(z)=\hat{q}_{s} \circ \tau_{s}\left(1+z^{2}\right)$. Since $l_{s}\left(i\left[-\varepsilon_{0}, \varepsilon_{0}\right]\right)$ $\subset S(0,1)$ and the range of the semi-disk by $\hat{q}_{s}$ is contained in a ring $\overline{D(0, M)}-D(0,1 / M)$, we may regard $l_{s}$ as an analytic function in $D\left(0, \varepsilon_{0}\right)$ whose range is contained in the ring. Thus $\left\{l_{s}\right\}_{0<s<1}$ is a normal family of analytic functions in $D\left(0, \varepsilon_{0}\right)$. Lemma 15 shows that $\left\{\hat{q}_{s}\right\}_{0<s<1}$ converges to $\hat{q}_{0}$ in $D\left(\varepsilon_{0} / 2, \varepsilon_{0} / 4\right)$, which yields that $\left\{l_{s}\right\}_{0<s<1}$ converges to $l_{0}$ in $D\left(0, \varepsilon_{0}\right)$. Thus $\lim _{s \downarrow 0} l_{s}^{\prime}(0)=l_{0}^{\prime}(0)$. Since

$$
\hat{q}_{s} \circ \tau_{s}(1+\delta)=\hat{q}_{s} \circ \tau_{s}(1)+\alpha_{s} \sqrt{\delta}+o(\sqrt{\delta}) \quad(\delta \downarrow 0),
$$

we have

$$
l_{s}(z)=l_{s}(0)+\alpha_{s} z+o(|z|) \quad(z \rightarrow 0),
$$

and hence $\alpha_{s}=l_{s}^{\prime}(0)(0 \leq s<1)$. Thus

$$
\lim _{s \downarrow 0} \alpha_{s}=\lim _{s \downarrow 0} l_{s}^{\prime}(0)=l_{0}^{\prime}(0)=\alpha_{0} .
$$

Lemma 17. Let

$$
\beta_{t}=\lim _{u \downarrow t}\left\{q\left(w_{u} ; \Lambda_{t}^{c}\right)-q\left(w_{t} ; \Lambda_{t}^{c}\right)\right\} / \sqrt{u-t} \quad(0<t \leq|\Gamma|) .
$$

Then $\lim _{t \downarrow 0} \beta_{t}$ exists and the value is common for all $\Gamma \in \mathscr{L}_{\theta}$, i.e., the value depends only on $\theta$. If $\theta=\pi$, then $\lim _{t \downarrow 0} \beta_{t}=0$.

To prove this lemma, we write simply $\tilde{q}_{t}(z)=q\left(z ; \Lambda_{t}^{c}\right) \quad(0<t \leq|\Gamma|)$. Since $\Gamma \in \mathscr{L}_{\theta}$, we can express $\Gamma$ as $e^{i \theta} s+s^{3 / 2} \hat{P}(\sqrt{s}), 0 \leq s<r$ in a small disk $D(0, r)$ with an analytic function $\hat{P}$ in $D\left(0, r^{2}\right)$. Dilating the coordinate axes if necessary, we may assume that $r=1$. Let $\hat{w}_{s}=e^{i \theta} s+$ $s^{3 / 2} \hat{P}(\sqrt{s})(0 \leq s<1)$. This is a parametric representation of $\Gamma \cap D$ different, in general, from $w_{t}$. Define $t=\tilde{t}(s)$ by $w_{t}=\hat{w}_{s}$. Then $d t / d s$ is 
right-continuous at $s=0$ and $\tilde{t}^{\prime}(0)=1$. We have $\hat{\beta}_{s}=\beta_{t} \sqrt{d t / d s}$ with

$$
\hat{\beta}_{s}=\lim _{\varepsilon \downarrow 0}\left\{\tilde{q}_{t}\left(\hat{w}_{s+\varepsilon}\right)-\tilde{q}_{t}\left(\hat{w}_{s}\right)\right\} / \sqrt{\varepsilon} \quad(t=\tilde{t}(s)) .
$$

Thus it is sufficient to show that $\left\{\hat{\beta}_{s}\right\}_{0<s<1}$ converges to a quantity depending only on $\theta$. Take a conformal mapping

$$
Q(z)=i \frac{q\left(z ;[-1,0]^{c}\right)-1}{q\left(z ;[-1,0]^{c}\right)+1}
$$

from $[-1,0]^{c}$ onto the upper halfplane $\mathbf{C}_{+}$. Then $Q(0)=0$ and $Q(\infty)=i$. We can write $Q(z)=i \sqrt{z}+z P^{*}(\sqrt{z})$ with an analytic function $P^{*}$ in D, and hence we can write

$$
i\left(\hat{w}_{s}\right)^{1 / 2}+\hat{w}_{s} P^{*}\left(\left(\hat{w}_{s}\right)^{1 / 2}\right)=i e^{i \theta / 2} \sqrt{s}+s P(\sqrt{s})
$$

with an analytic function $P$ in $\mathbf{D}$. Thus $Q(\Gamma \cap \mathbf{D})$ is expressed as $i e^{i \theta / 2} y$ $+y^{2} P(y), 0 \leq y<1$. Let $U_{s}, V_{s}, \tau_{s}, \alpha_{s}, \hat{q}_{s}=q\left(\cdot ; V_{s}^{c}\right)(0 \leq s<1)$ be the same as in Lemmas 15 and 16 . Then $Q\left(\Gamma_{t}\right)=\sqrt{s} U_{s}(t=\tilde{t}(s),(0 \leq s<1)$. Since $\hat{q}_{s}^{\prime}(\infty)>0$ and $V_{s}$ is symmetric with respect to the real line $\mathbf{R}, \hat{q}_{s}$ is a conformal mapping from $\mathbf{C}_{+}-U_{s}$ onto $\mathbf{C}_{+}-\left\{\mathbf{C}_{+} \cap \overline{\mathbf{D}}\right\}$, and hence

$$
m_{s}(z)=\frac{1}{2}\left\{\hat{q}_{s}(Q(z) / \sqrt{s})+\hat{q}_{s}(Q(z) / \sqrt{s})^{-1}\right\}
$$

is a conformal mapping from $\Lambda_{t}^{c}(t=\tilde{t}(s))$ onto $\mathbf{C}_{+}$. Thus

$$
\left\{m_{s}(z)-\overline{m_{s}(\infty)}\right\} /\left\{m_{s}(z)-m_{s}(\infty)\right\}
$$

is a conformal mapping from $\Lambda_{t}^{c}$ onto $\overline{\mathbf{D}}^{c}$, and hence

$$
\tilde{q}_{t}(z)=e^{i \phi_{s}}\left\{m_{s}(z)-\overline{m_{s}(\infty)}\right\} /\left\{m_{s}(z)-m_{s}(\infty)\right\} \quad(t=\tilde{t}(s))
$$

for some $0 \leq \phi_{s}<2 \pi$. Since $Q\left(\hat{w}_{s+\varepsilon}\right) / \sqrt{s}=\tau_{s}(\sqrt{1+(\varepsilon / s)})$, we have

$$
\begin{aligned}
\tilde{q}_{t}\left(\hat{w}_{s+\varepsilon}\right)-\tilde{q}_{t}\left(\hat{w}_{s}\right)= & -\frac{e^{i \phi_{s}} \frac{\left\{m_{s}(\infty)-\overline{m_{s}(\infty)}\right\}\left\{m_{s}\left(\hat{w}_{s+\varepsilon}\right)-m_{s}\left(\hat{w}_{s}\right)\right\}}{\left\{m_{s}\left(\hat{w}_{s+\varepsilon}\right)-m_{s}(\infty)\right\}\left\{m_{s}\left(\hat{w}_{s}\right)-m_{s}(\infty)\right\}}}{=} \\
& -e^{i \phi_{s}} \frac{\left\{m_{s}(\infty)-\overline{m_{s}(\infty)}\right\}\left\{1-\left[\hat{q}_{s} \circ \tau_{s}(\sqrt{1+(\varepsilon / s)}) \hat{q}_{s} \circ \tau_{s}(1)\right]^{-1}\right\}}{2\left\{m_{s}\left(\hat{w}_{s+\varepsilon}\right)-m_{s}(\infty)\right\}\left\{m_{s}\left(\hat{w}_{s}\right)-m_{s}(\infty)\right\}} \\
& \times\left\{\hat{q}_{s} \circ \tau_{s}(\sqrt{1+(\varepsilon / s)})-\hat{q}_{s} \circ \tau_{s}(1)\right\} \quad(t=\tilde{t}(s)) .
\end{aligned}
$$

Thus

$$
\hat{\beta}_{s}=-e^{i \phi_{s}} \frac{\alpha_{s}\left\{m_{s}(\infty)-\overline{m_{s}(\infty)}\right\}\left\{1-\hat{q}_{s} \circ \tau_{s}(1)^{-2}\right\}}{2 \sqrt{2 s}\left\{m_{s}\left(\hat{w}_{s}\right)-m_{s}(\infty)\right\}^{2}} .
$$


To study the right-hand side, we write

$$
\hat{q}_{s}(\zeta)=\gamma\left(V_{s}\right)^{-1} \zeta+\sum_{n=0}^{\infty} a_{s, n} \zeta^{-n} \quad(\zeta \rightarrow \infty) .
$$

Then $\left|a_{s, n}\right| \leq M^{n}(n=0,1, \cdots ; 0<s<1)$ for some $M>1$. Lemma 15 shows that

$$
\lim _{s \downarrow 0} \sqrt{s} m_{s}(\infty)=\lim _{s \downarrow 0} \frac{\sqrt{s}}{2}\left\{\hat{q}_{s}(i / \sqrt{s})+\hat{q}_{s}(i / \sqrt{s})^{-1}\right\}=\frac{i}{2} \gamma\left(V_{0}\right)^{-1} .
$$

Since $Q(z)=i-i /(2 z)+O\left(|z|^{-2}\right)(z \rightarrow \infty)$, we have

$$
\begin{aligned}
\hat{q}_{s}(Q(z) / \sqrt{s})= & \gamma\left(V_{s}\right)^{-1}(i / \sqrt{s})\left\{1-\frac{1}{2 z}+O\left(|z|^{-2}\right)\right\} \\
& +\sum_{n=0}^{\infty} a_{s, n}(-i)^{n} s^{n / 2}\left\{1-\frac{1}{2 z}+O\left(|z|^{-2}\right)\right\}^{-n} \\
= & \hat{q}_{s}(i / \sqrt{s})-\left\{\frac{i}{2 \sqrt{s}} \gamma\left(V_{s}\right)^{-1}-\sum_{n=1}^{\infty} \frac{n}{2} a_{s, n}(-i)^{n} s^{n / 2}\right\} \frac{1}{z}+\begin{array}{r}
O\left(|z|^{-2}\right) \\
(z \rightarrow \infty),
\end{array}
\end{aligned}
$$

and hence

$$
\begin{aligned}
\tilde{q}_{t}^{\prime}(\infty)= & e^{i \phi_{s}}\left\{m_{s}(\infty)-\overline{m_{s}(\infty)}\right\} \lim _{z \rightarrow \infty} \frac{1}{z\left\{m_{s}(z)-m_{s}(\infty)\right\}} \\
= & 2 e^{i \phi_{s}} \frac{m_{s}(\infty)-\overline{m_{s}(\infty)}}{1-\hat{q}_{s}(i / \sqrt{s})^{-2}} \lim _{z \rightarrow \infty} \frac{1}{z\left\{\hat{q}_{s}(Q(z) / \sqrt{s})-\hat{q}_{s}(i / \sqrt{s})\right\}} \\
= & -2 e^{i \phi_{s}} \frac{m_{s}(\infty)-\overline{m_{s}(\infty)}}{1-\hat{q}_{s}(i / \sqrt{s})^{-2}} \\
& \times\left\{\frac{i}{2 \sqrt{s}} \gamma\left(V_{s}\right)^{-1}-\sum_{n=1}^{\infty} \frac{n}{2} a_{s, n}(-i)^{n} s^{n / 2}\right\}^{-1} \quad(t=\tilde{t}(s)) .
\end{aligned}
$$

Since $\lim _{t \downarrow 0} \tilde{q}_{t}^{\prime}(\infty)=4$ and $\lim _{s \downarrow 0} \sqrt{s} m_{s}(\infty)=i_{\gamma}\left(V_{0}\right)^{-1} / 2$, we have $\lim _{s ! 0} e^{i \phi_{s}}=-1$. Consequently, Lemma 16 and (44) yield

$$
\lim _{s \downarrow 0} \hat{\beta}_{s}=-\sqrt{2} i \alpha_{0} \gamma\left(V_{0}\right)\left\{1-\hat{q}_{0} \circ \tau_{0}(1)^{-2}\right\} .
$$

The expression in the right-hand side shows that $\lim _{s \downarrow 0} \hat{\beta}_{s}$ depends only on $\theta$, i.e., $\lim _{t \downarrow 0} \beta_{t}$ depends only on $\theta$. If $\theta=\pi$, then $\hat{q}_{0} \circ \tau_{0}(1)=-1$, which gives $\lim _{s \downarrow 0} \hat{\beta}_{s}=0$, i.e., $\lim _{t \downarrow 0} \beta_{t}=0$.

Lemma 18. Let $n_{t}$ be the conformal mapping from $\Lambda_{t}^{c}$ onto $\left[-1, \delta_{t}\right]^{c}$ $\left(\delta_{t}>0\right)$ such that $n_{t}(\infty)=\infty,\left|n_{t}^{\prime}(\infty)\right|=1$ and $n_{t}\left(w_{t}\right)=\delta_{t}(0<t \leq|\Gamma|)$. Then 


$$
\lim _{t \downarrow 0}\left|n_{t}^{\prime}\left(w_{t}\right)\right|=\lim _{t \downarrow 0} \delta_{t} / t=\left(\frac{\pi-\theta}{\pi+\theta}\right)^{\theta / \pi} .
$$

Suppose that $0<\theta<\pi$. We begin by showing that $\lim _{t \downarrow 0} \delta_{t} / t$ exists and depends only on $\theta$. Recall $\tilde{q}_{t}(z)=q\left(z ; \Lambda_{t}^{c}\right)(0 \leq t \leq|\Gamma|)$. The function $\tilde{q}_{t}\left(w_{t}\right)^{-1} \tilde{q}_{t}(z)+\tilde{q}_{t}\left(w_{t}\right) \tilde{q}_{t}(z)^{-1}$ maps $\Lambda_{t}^{c}$ onto $[-2,2]^{c}$ so that the image of $w_{t}$ is 2. Thus

$$
n_{t}(z)=\gamma\left(\Lambda_{t}\right)\left\{\tilde{q}_{t}\left(w_{t}\right)^{-1} \tilde{q}_{t}(z)+\tilde{q}_{t}\left(w_{t}\right) \tilde{q}_{t}(z)^{-1}+2\right\}-1,
$$

which shows that $\delta_{t}=4 \gamma\left(\Lambda_{t}\right)-1$. Since $\tilde{q}_{t}^{\prime}(\infty)=1 / \gamma\left(\Lambda_{t}\right)$, (46) shows that

$$
\gamma\left(\Lambda_{t}\right)=\frac{\left|1-\hat{q}_{s}(i / \sqrt{s})^{-2}\right|}{2 \mid m_{s}(\infty)-\overline{m_{s}(\infty) \mid}}\left|\frac{i}{2 \sqrt{s}} \gamma\left(V_{s}\right)^{-1}-\sum_{n=1}^{\infty} \frac{n}{2} a_{s, n}(-i)^{n} s^{n / 2}\right| \quad(t=\tilde{t}(s)),
$$

where $\hat{q}_{s}, m_{s}, \tilde{t}(s)$ are the functions in the proof of Lemma 17. Recall (45). Since $\hat{q}_{s}^{\prime}(\infty)>0$ and $V_{s}$ is symmetric with respect to $\mathbf{R}, a_{s, n}(n=0,1)$ are real-valued, and hence

$$
\begin{aligned}
& \sqrt{s}\left|m_{s}(\infty)-\overline{m_{s}(\infty)}\right| \\
& =\frac{\sqrt{s}}{2}\left|\hat{q}_{s}(i / \sqrt{s})-\overline{\hat{q}_{s}(i / \sqrt{s})}+\hat{q}_{s}(i / \sqrt{s})^{-1}-\overline{\hat{q}_{s}(i / \sqrt{s})^{-1}}\right| \\
& =\gamma\left(V_{s}\right)^{-1}-\left\{a_{s, 1}+\gamma\left(V_{s}\right)\right\} s+o(s) \quad(s \downarrow 0) \text {. }
\end{aligned}
$$

Thus

$$
\begin{aligned}
\gamma\left(\Lambda_{t}\right) & =\frac{1}{4} \frac{\left\{1+\gamma\left(V_{s}\right)^{2} s\right\}\left\{\gamma\left(V_{s}\right)^{-1}+a_{s, 1} s\right\}}{\gamma\left(V_{s}\right)^{-1}-\left\{a_{s, 1}+\gamma\left(V_{s}\right)\right\} s}+o(s) \\
& =\frac{1}{4}+\frac{1}{2} \gamma\left(V_{s}\right)\left\{\gamma\left(V_{s}\right)+a_{s, 1}\right\} s+o(s) \quad(t=\tilde{t}(s), s \downarrow 0) .
\end{aligned}
$$

Since $\left\{\hat{q}_{s}\right\}_{0<s<1}$ converges to $\hat{q}_{0}$, we have $\lim _{s ! 0} a_{s, 1}=a_{0,1}$. Consequently,

$$
\begin{aligned}
\lim _{t ! 0} \delta_{l} / t & =\lim _{t \downarrow 0}\left\{4 \gamma\left(\Lambda_{t}\right)-1\right\} / t \\
& =\lim _{s \downarrow 0} \frac{2 \gamma\left(V_{s}\right)\left\{\gamma\left(V_{s}\right)+a_{s, 1}\right\}}{\tilde{t}^{\prime}(s)}=2 \gamma\left(V_{0}\right)\left\{\gamma\left(V_{0}\right)+a_{0,1}\right\} .
\end{aligned}
$$

The last expression shows that $\lim _{t \downarrow 0} \delta_{t} / t$ depends only on $\theta$. Next we show that $\lim _{t \downarrow 0}\left|n_{t}^{\prime}\left(w_{t}\right)\right|$ exists and depends only on $\theta$. Since

$$
n_{t}\left(w_{u}\right)-n_{t}\left(w_{t}\right)=\gamma\left(V_{t}\right) \tilde{q}_{t}\left(w_{u}\right)^{-1} \tilde{q}_{t}\left(w_{t}\right)^{-1}\left\{\tilde{q}_{t}\left(w_{u}\right)-\tilde{q}_{t}\left(w_{t}\right)\right\}^{2} \quad(0<t<u),
$$

we have

$$
n_{t}^{\prime}\left(w_{t}\right)=\left(\frac{d w_{t}}{d t}\right)^{-1} \lim _{u \downarrow t} \frac{n_{t}\left(w_{u}\right)-n_{t}\left(w_{t}\right)}{u-t}=\gamma\left(V_{t}\right)\left(\frac{d w_{t}}{d t}\right)^{-1} \tilde{q}_{t}\left(w_{t}\right)^{-2} \beta_{t}^{2}
$$


Thus

$$
\lim _{t \downarrow 0}\left|n_{t}^{\prime}\left(w_{t}\right)\right|=\gamma\left(V_{0}\right) \lim _{t \downarrow 0}\left|\beta_{t}\right|^{2} .
$$

Lemma 17 shows that $\lim _{t \downarrow 0}\left|n_{t}^{\prime}\left(w_{t}\right)\right|$ depends only on $\theta$. Since both $\lim _{t \downarrow 0}\left|n_{t}^{\prime}\left(w_{t}\right)\right|$ and $\lim _{t \downarrow 0} \delta_{t} / t$ depend only on $\theta$, it is sufficient to prove (47) in the case $\Gamma=e^{i \theta}[0,1]$. For $0<\varepsilon<1$, we take the Schwarz-Christoffel transformation

$$
\rho_{\varepsilon}(z)=\frac{1}{z}\left(z-e^{-i \varepsilon}\right)^{1+\phi}\left(z-e^{i \varepsilon}\right)^{1-\phi} \quad(\phi=\theta / \pi)
$$

from $\overline{\mathbf{D}}^{c}$ onto a domain $F_{\varepsilon}^{c}$ of form

$$
e^{i \Downarrow \varepsilon} F_{\varepsilon}=\left[-\sigma_{\varepsilon}, 0\right] \cup\left(e^{i \theta}\left[0, \eta_{\varepsilon}\right]\right)
$$

for some triple $\left(\psi_{\varepsilon}, \sigma_{\varepsilon}, \eta_{\varepsilon}\right)$ of non-negative numbers. Let $a$ be the number in $(-\varepsilon, \varepsilon)$ such that $\left.\frac{\partial}{\partial x}\left|\rho_{\varepsilon}\left(e^{i x}\right)\right|\right|_{x=a}=0$. Then

$$
(1+\phi) \tan \frac{\varepsilon-\mathfrak{a}}{2}=(1-\phi) \tan \frac{\varepsilon+\mathfrak{a}}{2},
$$

and hence $\mathfrak{a}$ is analytic in a neighborhood of 0 with respect to $\varepsilon$ and $\mathfrak{a}=\phi \varepsilon+O\left(\varepsilon^{3}\right)(\varepsilon \downarrow 0)$. Since $\rho_{\varepsilon}\left(e^{ \pm i \varepsilon}\right)=0$, we have

$$
\eta_{\varepsilon}=\left|\rho_{\varepsilon}\left(e^{i \mathfrak{a}}\right)\right|=4 \sin ^{1+\phi} \frac{\varepsilon+\mathfrak{a}}{2} \sin ^{1-\phi} \frac{\varepsilon-\mathfrak{a}}{2},
$$

and hence $\eta_{\varepsilon}$ is analytic in a neighborhood of 0 with respect to $\varepsilon$ and

$$
\eta_{\varepsilon}=(1+\phi)^{1+\phi}(1-\phi)^{1-\phi} \varepsilon^{2}+O\left(\varepsilon^{4}\right) \quad(\varepsilon \downarrow 0) .
$$

Let $\mathfrak{b}$ be the number in $(-\varepsilon, \varepsilon)$ such that $\left.\frac{\partial}{\partial x}\left|\rho_{\varepsilon}\left(-e^{i x}\right)\right|\right|_{x=6}=0$. Then

$$
(1+\phi) \tan \frac{\varepsilon+\mathfrak{b}}{2}=(1-\phi) \tan \frac{\varepsilon-\mathfrak{b}}{2},
$$

and hence $\mathfrak{b}$ is analytic in a neighborhood of 0 with respect to $\varepsilon$ and $\mathfrak{b}=-\phi \varepsilon+O\left(\varepsilon^{3}\right)(\varepsilon \downarrow 0)$. We have

$$
\sigma_{\varepsilon}=\left|\rho_{\varepsilon}\left(-e^{i \mathfrak{b}}\right)\right|=4 \cos ^{1+\phi} \frac{\varepsilon+\mathfrak{b}}{2} \cos ^{1-\phi} \frac{\varepsilon-\mathfrak{b}}{2},
$$

and hence $\sigma_{\varepsilon}$ is analytic in a neighborhood of 0 with respect to $\varepsilon$ and 


$$
\sigma_{\varepsilon}=4-(1+\phi)(1-\phi) \varepsilon^{2}+O\left(\varepsilon^{4}\right) \quad(\varepsilon \downarrow 0) .
$$

Considering a conformal mapping $e^{i \psi \varepsilon} \sigma_{\varepsilon}^{-1} \rho_{\varepsilon}(z)$ from $\bar{D}^{c}$ onto $\left\{[-1,0] \cup\left(e^{i \theta}\left[0, \eta_{\varepsilon} \sigma_{\varepsilon}^{-1}\right]\right)\right\}^{c}$, we obtain

$$
\gamma\left([-1,0] \cup\left(e^{i \theta}\left[0, \eta_{\varepsilon} \sigma_{s}^{-1}\right]\right)\right)=\sigma_{\varepsilon}^{-1} .
$$

Given a small number $t>0$, we define $\varepsilon=\varepsilon(t)$ by

$$
t=\eta_{\varepsilon} \sigma_{\varepsilon}^{-1}=\frac{1}{4}(1+\phi)^{1+\phi}(1-\phi)^{1-\phi} \varepsilon^{2}+\cdots
$$

Since

$$
\begin{aligned}
\sigma_{\varepsilon}^{-1} & =\frac{1}{4}+\frac{(1+\phi)(1-\phi)}{16} \varepsilon^{2}+\cdots \\
& =\frac{1}{4}+\frac{1}{4}\left(\frac{1-\phi}{1+\phi}\right)^{\phi} t+o(t) \quad(t \downarrow 0),
\end{aligned}
$$

we have

$$
\lim _{t \downarrow 0} \delta_{t} / t=4 \lim _{t \downarrow 0} \frac{1}{t}\left\{\gamma\left(\Lambda_{t}\right)-\gamma([-1,0])\right\}=\left(\frac{1-\phi}{1+\phi}\right)^{\phi},
$$

which shows the second equality in (47). Since $\delta_{t}$ is analytic in a neighborhood of 0 with respect to $t$, we have

$$
\lim _{t \downarrow 0} \frac{d \delta_{t}}{d t}=4 \lim _{t \downarrow 0} \frac{\partial \gamma}{\partial t}\left(\Lambda_{t}\right)=\left(\frac{1-\phi}{1+\phi}\right)^{\phi} .
$$

We can write

$$
\begin{aligned}
n_{t}\left(w_{u}\right) & =\delta_{t}+n_{t}^{\prime}\left(w_{t}\right)\left(w_{u}-w_{t}\right)+O\left(\left|w_{u}-w_{t}\right|^{3 / 2}\right) \\
& =\delta_{t}+n_{t}^{\prime}\left(w_{t}\right) \frac{d w_{t}}{d t}(u-t)+O\left((u-t)^{3 / 2}\right) \quad(u \downarrow t)
\end{aligned}
$$

with $n_{t}^{\prime}\left(w_{t}\right) d w_{t} / d t>0$. Since $\left|n_{t}^{\prime}(\infty)\right|=1$, Lemma 11 shows that

$$
\begin{aligned}
\gamma\left(\Lambda_{u}\right)-\gamma\left(\Lambda_{t}\right) & =\gamma\left(n_{t}\left(\Lambda_{u}\right)\right)-\gamma\left(\left[-1, \delta_{t}\right]\right) \\
& =\frac{1}{4}\left|n_{t}^{\prime}\left(w_{t}\right)\right|(u-t)+o(u-t) \quad(u \downarrow t),
\end{aligned}
$$

which gives that $d \delta_{t} / d t=\left|n_{t}^{\prime}\left(w_{t}\right)\right|$. Combined with (49), the first equality in (47) follows. In the same manner as above, we obtain (47) in the case where $-\pi<\theta<0$. Suppose that $\theta=\pi$. Since $\Gamma \in \mathscr{L}_{\pi}$, we have 
$\Lambda_{t} \subset\left\{x+i y ;-1 \leq x \leq 0,|y| \leq C t^{3 / 2}\right\}(t \downarrow 0)$ for some constant $C$. Since $\gamma\left(\left\{x+i y ;-1 \leq x \leq 0,|y| \leq C t^{3 / 2}\right\}\right)=1 / 4+o(t) \quad(t \downarrow 0)$, we have $\lim _{t \downarrow 0} \delta_{t} / t$ $=0$. Since $\lim _{t \downarrow 0} \beta_{t}=0$, (48) yields that $\lim _{t \downarrow 0}\left|n_{t}^{\prime}\left(w_{t}\right)\right|=0$. This completes the proof of Lemma 18.

Now we return to the proof of the existence of $\partial K\left(z, \bar{\zeta} ; \Omega_{0}\right) / \partial t$ and the continuity of $\partial K\left(z, \bar{\zeta} ; \Omega_{t}\right) / \partial t$ at $t=0$ (in the case where $\Lambda=[-1,0]$ and $\Gamma \in \mathscr{L}_{\theta}, \theta \neq 0$.) Let $n_{t}$ be the conformal mapping in Lemma 18 $(0<t \leq|\Gamma|)$. Then

$$
\begin{aligned}
& \frac{\partial K}{\partial t}\left(z, \zeta ; \Omega_{t}\right)=\left.\frac{\partial K}{\partial \varepsilon}\left(n_{t}(z), \overline{n_{t}(\zeta)} ; n_{t}\left(\Omega_{t+\varepsilon}\right)\right)\right|_{\varepsilon=0} \sqrt{n_{t}^{\prime}(z)} \sqrt{\sqrt{n_{t}^{\prime}(\zeta)}} \\
& =\left.\frac{\partial K}{\partial \varepsilon}\left(n_{t}(z), \overline{n_{t}(\zeta)} ; n_{\iota}\left(\Omega_{t}\right)-[0, \varepsilon]\right)\right|_{\iota=\bar{o}_{t}}\left|n_{t}^{\prime}\left(w_{t}\right)\right| \sqrt{n_{t}^{\prime}(z)} \sqrt{n_{t}^{\prime}(\zeta)} \\
& (0<t \leq|\Gamma|) .
\end{aligned}
$$

The argument in the case I shows that

$$
\left.\lim _{\iota, 0} \frac{\partial K}{\partial \varepsilon}\left(n_{t}(z), \overline{n_{t}(\bar{\zeta})} ; n_{l}\left(\Omega_{l}\right)-[0, \varepsilon]\right)\right|_{\varepsilon=\tilde{o}_{l}}=\left.\frac{\partial K}{\partial \varepsilon}(z, \zeta ; \Omega-[0, \varepsilon])\right|_{\iota=0},
$$

and hence Lemma 18 yields that

$$
\lim _{t \downarrow 0} \frac{\partial K}{\partial t}\left(z, \bar{\zeta} ; \Omega_{t}\right)=\left.\left(\frac{\pi-\theta}{\pi+\theta}\right)^{o / \pi} \frac{\partial K}{\partial \varepsilon}(z, \zeta ; \Omega-[0, \varepsilon])\right|_{\iota=0} .
$$

Let $n_{t}^{*}$ be the conformal mapping from $\Lambda_{\iota}^{c}$ onto $\left[-1, \delta_{t}\right]^{c}$ such that $n_{t}^{*}(\infty)$ $=\infty$ and $n_{t}^{* \prime}(\infty)=1(0<t \leq|\Gamma|)$. (Note that $n_{t}^{*}\left(w_{t}\right) \neq \delta_{t}$.) Lemma 12 shows that $n_{t}^{* \prime}(z)=o(t)(t \downarrow 0, z \in \Omega-\Gamma)$. Thus

$$
\begin{aligned}
K\left(z, \bar{\zeta} ; \Omega_{t}\right) & =K\left(n_{t}^{*}(z), \overline{n_{t}^{*}(\zeta)} ; n_{t}^{*}\left(\Omega_{t}\right)\right) \sqrt{\overline{n_{t}^{* \prime}(z)} \sqrt{\sqrt{n_{t}^{* \prime}(\zeta)}}} \\
& =K\left(z, \bar{\zeta} ; \Omega-\left[0, \delta_{t}\right]\right)+o(t) \\
& =K(z, \bar{\zeta} ; \Omega)+\left.\delta_{t} \frac{\partial K}{\partial \varepsilon}(z, \bar{\zeta} ; \Omega-[0, \varepsilon])\right|_{\varepsilon=0}+o(t) \quad(t \downarrow 0) .
\end{aligned}
$$

By Lemma 18, we have

$$
\frac{\partial K}{\partial t}\left(z, \bar{\zeta} ; \Omega_{0}\right)=\left.\left(\frac{\pi-\theta}{\pi+\theta}\right)^{\theta / \pi} \frac{\partial K}{\partial \varepsilon}(z, \bar{\zeta} ; \Omega-[0, \varepsilon])\right|_{\varepsilon=0} .
$$

Consequently, $\partial K\left(z, \bar{\zeta} ; \Omega_{0}\right) / \partial t$ exists and $\partial K\left(z, \bar{\zeta} ; \Omega_{t}\right) / \partial t$ is continuous at $t=0$. This completes the proof of Theorem 8 in the case IV.

Remark 19. We easily see that $\lim _{\iota \downarrow 0} D c\left(w_{\iota}, \infty ; \Omega_{0}\right)\left(=D c\left(w_{0}, \infty ; \Omega_{0}\right)\right.$, 
say) exists even if $w_{0} \in \partial \Omega$. We here note that the right-derivative $\partial c\left(\infty ; \Omega_{0}\right) / \partial t$ at $t=0$ is not, in general, equal to $D c\left(w_{0}, \infty ; \Omega_{0}\right) / 4$. Take $\Omega=[-1,0]^{c}$ and $\Gamma=e^{i \theta}[0,1](0<\theta<\pi)$, for example. Then the second equality in (47) shows that

$$
\frac{\partial c}{\partial t}\left(\infty ; \Omega_{0}\right)=\frac{1}{4}\left(\frac{\pi-\theta}{\pi+\theta}\right)^{\theta / \pi} .
$$

We have

$$
D c\left(w_{\iota}, \infty ; \Omega_{0}\right)=\cos \left\{\frac{1}{2} \operatorname{Im} \int_{-1}^{0} \frac{d x}{x-w_{t}}\right\}=\cos \left\{\frac{1}{2} \psi\left(w_{t}\right)\right\},
$$

where $\psi\left(w_{t}\right)$ is the angle at $w_{t}$ of the triangle with vertexes $-1,0, w_{t}$. (See Proposition 24.) Letting $t$ tend to 0 , we obtain

$$
D c\left(w_{0}, \infty ; \Omega_{0}\right)=\cos \frac{\theta}{2} \text {. }
$$

Since $\{(\pi-\theta) /(\pi+\theta)\}^{\theta / \pi} \neq \cos (\theta / 2)$, we have $\partial c\left(\infty ; \Omega_{0}\right) / \partial t \neq D c\left(w_{0}, \infty ; \Omega_{0}\right) / 4$.

\section{§ 8. Application to simply-connected domains}

In this section, we mainly study $D^{2} c(w, z, \zeta ; \Omega)$ for simply-connected domains $\Omega$. Prior to the study, we give some remarks to $D c(z, \infty ; \Omega)$ and $D^{2} c(z, \zeta, \infty ; \Omega)$ for general multiply-connected domains $\Omega$. Let $h$ be a conformal mapping from a domain $\Omega$ containing $\infty$ onto a radial slit domain $\left[21\right.$, p. 335] $\Omega^{*}$ such that $h(\infty)=\infty,\left|h^{\prime}(\infty)\right|=1$. Then $D c\left(z_{0}, \infty ; \Omega\right)$ $\leq D c\left(0, \infty ; \Omega^{*}\right)$, where $z_{0}$ is the point in $\Omega$ such that $h\left(z_{0}\right)=0$. In fact, Rengel's inequality [33, p. 393] shows that $\left|h^{\prime}\left(z_{0}\right)\right| \leq 1$. Since $\operatorname{Dc}(z, \zeta ; \Omega)$ $\times|d z||d \zeta|$ is conformally invariant, we obtain the required inequality. If $\Omega^{*}$ is a circular slit domain $\left[21\right.$, p. 335], we have $D c\left(z_{0}, \infty ; \Omega\right) \geq D c\left(0, \infty ; \Omega^{*}\right)$. Thus radial slit domains and circular slit domains are very important to estimate $D c(z, \infty ; \Omega)$. A simple calculation shows that $D c(z, \infty ; \Omega)$ is superharmonic in $\Omega$ and, if $\Omega$ is bounded by analytic Jordan curves, then $D c(z, \infty ; \Omega)=0$ on $\partial \Omega$. The behaviour of $D^{2} c(z, \zeta, \infty ; \Omega)$ near $z=\zeta$ is as follows. Recall the definition of $D^{2} c(z, \zeta, \infty ; \Omega)$. We can rewrite this quantity as

$$
\begin{aligned}
D^{2} c(z, \zeta, \infty ; \Omega)= & 2 \operatorname{Re}[\{-\phi(z ; \Omega) g(\zeta ; \Omega)+g(z ; \Omega) \phi(\zeta ; \Omega)\} \overline{L(z, \zeta ; \Omega)} \\
& -\{\phi(z ; \Omega) \overline{\phi(\zeta ; \Omega)}-g(z ; \Omega) \overline{g(\zeta ; \Omega)}\} \overline{K(z, \bar{\zeta} ; \Omega)}]
\end{aligned}
$$

Since the Ahlfors function $f(z ; \Omega)$ of $\Omega^{c}$ equals $g(z ; \Omega) / \phi(z ; \Omega)$, we have 


$$
\begin{aligned}
\lim _{\varepsilon \downarrow 0} D^{2} c & \left(\zeta+\varepsilon e^{i \theta}, \zeta, \infty ; \Omega\right)=2 \operatorname{Re}\left[\operatorname { l i m } _ { s \downarrow 0 } \left\{-\phi\left(\zeta+\varepsilon e^{i \theta} ; \Omega\right) g(\zeta ; \Omega)\right.\right. \\
& \left.\left.+g\left(\zeta+\varepsilon e^{i \theta} ; \Omega\right) \phi(\zeta ; \Omega)\right\} \frac{e^{i \theta}}{\varepsilon}-\left\{|\phi(\zeta ; \Omega)|^{2}-|g(\zeta ; \Omega)|^{2}\right\} \overline{K(\zeta, \zeta ; \Omega)}\right] \\
= & 2 \operatorname{Re}\left[e^{2 i \theta}\left\{-\phi^{\prime}(\zeta ; \Omega) g(\zeta ; \Omega)+g^{\prime}(\zeta ; \Omega) \phi(\zeta ; \Omega)\right\}\right. \\
& \left.-|\phi(\zeta ; \Omega)|^{2}\left\{1-|f(\zeta ; \Omega)|^{2}\right\} K(\zeta, \zeta ; \Omega)\right] \\
= & 2|\phi(\zeta ; \Omega)|^{2}\left\{1-|f(\zeta ; \Omega)|^{2}\right\} \\
& \times \operatorname{Re}\left(e^{2 i \theta} \frac{\phi(\zeta ; \Omega)}{\overline{\phi(\zeta ; \Omega)}} \frac{f^{\prime}(\zeta ; \Omega)}{1-|f(\zeta ; \Omega)|^{2}}-K(\zeta, \bar{\zeta} ; \Omega)\right) \\
(= & D_{\theta}^{2} c(\zeta, \zeta, \infty ; \Omega), \text { say) }(0 \leq \theta<2 \pi) .
\end{aligned}
$$

Note that $K(\zeta, \zeta ; \Omega)$ is the supremum of $\left|f^{\prime}(\zeta)\right|$ over all $f \in H^{\infty}(\Omega)$, $\|f\|_{H^{\infty}} \leq 1$. The function $\{f(z ; \Omega)-f(\zeta ; \Omega)\} /\{1-f(z ; \Omega) \overline{f(\zeta ; \Omega)}\}$ belongs to $H^{\infty}(\Omega)$, the norm is equal to 1 and the derivative at $z=\zeta$ is equal to $f^{\prime}(\zeta ; \Omega)$ $\times\left\{1-|f(\zeta ; \Omega)|^{2}\right\}^{-1}$. Thus $D_{\theta}^{2} c(\zeta, \zeta, \infty ; \Omega) \leq 0$. Since $D_{\theta}^{2} c(\zeta, \zeta, \infty ; \Omega)$ is not a constant with respect to $\theta, D^{2} c(z, \zeta, \infty ; \Omega)$ cannot be extended as a function in $(z, \zeta) \in \Omega \times \Omega$. If $\Omega$ is simply-connected, then there uniquely exists $0 \leq \theta<2 \pi$ such that $D_{\theta}^{2} c(\zeta, \zeta, \infty ; \Omega)=0$. In the case where $\Omega$ is simply-connected, it is easy to study $D^{2} c(w, z, \zeta ; \Omega)$. We have

Proposition 20. If $\Omega$ is simply-connected, then $D^{2} c(w, z, \zeta ; \Omega) \leq 0$. In particular,

$$
\begin{aligned}
& D^{2} c(0, z, \zeta ; \mathbf{D})=-\frac{(1+\mid z \zeta)^{4}|1+z \bar{\zeta}|^{2}}{\left(1-|z|^{2}\right)\left(1-|\zeta|^{2}\right)|z \zeta|^{2}|z-\zeta|^{2}} \\
& \quad \times\left\{\left(\frac{|z|+|\zeta|}{1+|z \zeta|}\right)^{2}-\left|\frac{z+\zeta}{1+z \zeta}\right|^{2}\right\}\left\{\left(\frac{|z|+|\zeta|}{1+|z \zeta|}\right)^{2}-\left|\frac{z-\zeta}{1-z \bar{\zeta}}\right|^{2}\right\}
\end{aligned}
$$

The equality $D^{2} c(0, z, \zeta ; \mathbf{D})=0$ holds if and only if $z \bar{\zeta}$ is real.

The hyperbolic distance in $\mathbf{D}$ is defined by

$$
d(z, \zeta)=\operatorname{arctanh}\left|\frac{z-\zeta}{1-z \bar{\zeta}}\right| \quad(z, \zeta \in \mathbf{D})
$$

We have

$$
d(z, 0)+d(0, \zeta)=\operatorname{arctanh} \frac{|z|+|\zeta|}{1+|z \zeta|} .
$$

Thus the expression in the right-hand side of (50) shows that $D^{2} c$ is closely related to the triangle inequality with respect to the hyperbolic distance. The proof of this proposition is as follows. By Proposition 2, 
we may assume that $\Omega=\mathbf{D}$ and $w=0$. Since

$$
K(z, \zeta ; \mathbf{D})=\frac{1}{1-z \zeta}, \quad L(z, \zeta ; \mathbf{D})=\frac{1}{z-\zeta} \quad[21, \text { p. 391] }
$$

we have

$$
\begin{aligned}
D^{2} c(w, z, \zeta ; \mathbf{D})= & 2 \operatorname{Re}\left[\left\{\frac{1}{w-z} \frac{1}{1-\bar{w} \zeta}-\frac{1}{1-\bar{w} z} \frac{1}{w-\zeta}\right\} \frac{1}{\bar{z}-\bar{\zeta}}\right. \\
& \left.-\left\{\frac{1}{w-z \bar{w}-\zeta}-\frac{1}{1-\bar{w} z} \frac{1}{1-w \bar{\zeta}}\right\} \frac{1}{1-\bar{z} \zeta}\right]
\end{aligned}
$$

and hence

$$
\begin{aligned}
& D^{2} c(0, z, \zeta ; \mathbf{D})=2 \operatorname{Re}\left\{\frac{z-\zeta}{z \zeta(\bar{z}-\bar{\zeta})}-\frac{1-z \bar{\zeta}}{z \bar{\zeta}(1-\bar{z} \zeta)}\right\} \\
&=\frac{1}{|z \zeta|^{2}|z-\zeta|^{2}}\left\{\left(|z|^{2}-|\zeta|^{2}\right)^{2}-|z-\zeta|^{2}\left(|z|^{2}+|\zeta|^{2}\right)\right\} \\
&-\frac{1}{|z \zeta|^{2}|1-z \bar{\zeta}|^{2}}\left\{\left(1-|z \zeta|^{2}\right)^{2}-|1-z \bar{\zeta}|^{2}\left(1+|z \zeta|^{2}\right)\right\} \\
&= \frac{1}{|z \zeta|^{2}}\left\{\frac{\left(|z|^{2}-|\zeta|^{2}\right)^{2}}{|z-\zeta|^{2}}-\frac{\left(1-|z \zeta|^{2}\right)^{2}}{|1-z \bar{\zeta}|^{2}}+\left(1-|z|^{2}\right)\left(1-|\zeta|^{2}\right)\right\} \\
&= \frac{1}{|z \zeta|^{2}}\left\{\frac{\left(|z|^{2}-|\zeta|^{2}\right)^{2}}{|z-\zeta|^{2}}-\frac{\left(1-|z \zeta|^{2}\right)^{2}}{|1-z \bar{\zeta}|^{2}}-\frac{\left(|z|^{2}-|\zeta|^{2}\right)^{2}}{(|z|-\mid \zeta)^{2}}+\frac{\left(1-|z \zeta|^{2}\right)^{2}}{(1-\mid z \zeta)^{2}}\right\} \\
&= \frac{\left(|z|^{2}-|\zeta|^{2}\right)^{2}}{|z \zeta|^{2}}\left\{\frac{1}{|z-\zeta|^{2}}-\frac{1}{(|z|-|\zeta|)^{2}}\right\} \\
&-\frac{\left(1-|z \zeta|^{2}\right)^{2}}{|z \zeta|^{2}}\left\{\frac{1}{|1-z \bar{\zeta}|^{2}}-\frac{1}{(1-|z \zeta|)^{2}}\right\} \\
&=-\frac{(|z|+|\zeta|)^{2}(2|z \zeta|-z \bar{\zeta}-\bar{z} \zeta)}{|z \zeta|^{2}|z-\zeta|^{2}}+\frac{(1+|z \zeta|)^{2}(2|z \zeta|-z \bar{\zeta}-\bar{z} \zeta)}{|z \zeta|^{2}|1-z \bar{\zeta}|^{2}} \\
&=-\frac{(2|z \zeta|-z \bar{\zeta}-\bar{z} \zeta)(1+|z \zeta|)^{2}}{|z \zeta|^{2}|z-\zeta|^{2}}\left\{\left(\frac{|z|+|\zeta|}{1+|z \zeta|}\right)^{2}-\mid \frac{z-\zeta}{\left.1-\left.z \bar{\zeta}\right|^{2}\right\} .}\right.
\end{aligned}
$$

A calculation shows that

$$
\begin{aligned}
& \left(\frac{|z|+|\zeta|}{1+|z \zeta|}\right)^{2}-\left|\frac{z+\zeta}{1+z \bar{\zeta}}\right|^{2}=\frac{(2|z \zeta|-z \bar{\zeta}-\bar{z} \zeta)\left(1-|z|^{2}\right)\left(1-|\zeta|^{2}\right)}{(1+\mid z \zeta)^{2}|1+z \bar{\zeta}|^{2}}, \text { i.e., } \\
& 2|z \zeta|-z \bar{\zeta}-\bar{z} \zeta=\frac{(1+|z \zeta|)^{2}|1+z \bar{\zeta}|^{2}}{\left(1-|z|^{2}\right)\left(1-|\zeta|^{2}\right)}\left\{\left(\frac{|z|+|\zeta|}{1+|z \zeta|}\right)^{2}-\left|\frac{z+\zeta}{1+z \bar{\zeta}}\right|^{2}\right\} .
\end{aligned}
$$

Replacing $2|z \zeta|-z \bar{\zeta}-\bar{z} \zeta$ by the expression in the right-hand side, we obtain (50). Equality (50) shows that $D^{2} c(0, z, \zeta ; \mathbf{D})=0$ if and only if 


$$
\text { "either } \frac{|z|+|\zeta|}{1+|z \zeta|}=\left|\frac{z+\zeta}{1+z \bar{\zeta}}\right| \text { or } \quad \frac{|z|+|\zeta|}{1+|z \zeta|}=\left|\frac{z-\zeta}{1-z \bar{\zeta}}\right| " \text {. }
$$

Thus $D^{2} c(0, z, \zeta ; \mathbf{D})=0$ if and only if $z \zeta$ is real. This completes the proof of Proposition 20.

Corollary 21. Let $\mathrm{Cap}(\cdot)$ denote the logarithmic capacity [35, p. 132]. Then for any two bounded continua $A$ and $B$ such that $A \cap B \neq \emptyset$,

$$
\operatorname{Cap}(A \cup B) \leq \operatorname{Cap}(A)+\operatorname{Cap}(B) .
$$

Note that $\operatorname{Cap}(\cdot)$ is not subadditive. If $E$ is connected, then $\operatorname{Cap}(E)$ $=\gamma(E)[35$, p. 132]. Hence it is sufficient to show that $\gamma(A \cup B) \leq \gamma(A)+$ $\gamma(B)$. Without loss of generality, we may assume that $A, B$ are bounded by Jordan curves, $A^{c}, B^{c} \in \mathscr{G}$ and $\partial A \cap \partial B$ consists of finite points. Let $w_{0} \in\left(\partial \Omega_{A \cup B}\right) \cap(\partial A \cap \partial B)$, where $\Omega_{A \cup B}$ is the component of $(A \cup B)^{c}$ containing $\infty$. For $0<\varepsilon<|\partial A|, A_{\varepsilon}$ denotes an arc with an endpoint $w_{0}$ such that $A_{\varepsilon} \subset \partial A$ and $\left|A_{\varepsilon}\right|=|\partial A|-\varepsilon$. We define $B_{\varepsilon}$ in the same manner. If $\gamma\left(A_{\varepsilon} \cup B_{\varepsilon}\right) \leq \gamma\left(A_{\varepsilon}\right)+\gamma\left(B_{\varepsilon}\right)$ for all sufficiently small numbers $\varepsilon>0$, then the required inequality is immediately deduced. Thus, from the beginning, we may assume that $A, B$ are arcs in $\mathscr{A}$ with a common endpoint $w_{0}$ contained in $\partial \Omega_{A \cup B}$ and $A \cap B$ consists of finite points. We define an arclength representation $\zeta_{t}(0 \leq t \leq|B|)$ of $B$ so that $\zeta_{0}=w_{0}$. Let $B_{t}=$ $\left\{\zeta_{s} ; 0 \leq s \leq t\right\} \quad(0 \leq t \leq|B|)$ and

$$
I=\left\{0 \leq t \leq|B| ; \zeta_{l} \in A \cap B \text { or } \zeta_{l} \notin \partial \Omega_{A \cup B_{l}}\right\} .
$$

We define a function on $[0,|B|]$ by

$$
D_{0} c\left(\zeta_{t}, \infty ; \Omega_{A \cup B_{t}}\right)= \begin{cases}D c\left(\zeta_{t}, \infty ; \Omega_{A \cup B_{t}}\right) & (t \in[0,|B|]-I) \\ 0 & (t \in I) .\end{cases}
$$

Then $D_{0} c\left(\zeta_{t}, \infty ; \Omega_{A \cup B_{t}}\right)$ is bounded on $[0,|B|]$. Since $\gamma\left(A \cup B_{t}\right)=\gamma\left(\Omega_{A \cup B_{t}}^{c}\right)$, we have

$$
\frac{\partial \gamma}{\partial t}\left(A \cup B_{t}\right)=0=\frac{1}{4} D_{0} c\left(\zeta_{t}, \infty ; \Omega_{A \cup B_{t}}\right) \quad\left(\zeta_{t} \notin \partial \Omega_{A \cup B_{t}}\right),
$$

and hence

$$
\gamma(A \cup B)=\gamma(A)+\frac{1}{4} \int_{0}^{|B|} D_{0} c\left(\zeta_{t}, \infty ; \Omega_{A \cup B_{t}}\right) d t
$$

We show that 
(51) $D_{0} c\left(\zeta_{t}, \infty ; \Omega_{A \cup B_{t}}\right) \leq D c\left(\zeta_{t}, \infty ; B_{t}^{c}\right) \quad(t \in[0,|B|])$.

If $t \in I$, (51) evidently holds. Suppose that $t \notin I$. Given $\varepsilon>0$, we can choose $\zeta_{t}^{*} \in \Omega_{A \cup B_{t}}$ so that

$$
\begin{aligned}
& \left|D c\left(\zeta_{t}, \infty ; \Omega_{A \cup B_{t}}\right)-D c\left(\zeta_{t}^{*}, \infty ; \Omega_{A \cup B_{t}}\right)\right|<\varepsilon, \\
& \left|D c\left(\zeta_{t}, \infty ; B_{t}^{c}\right)-D c\left(\zeta_{t}^{*}, \infty ; B_{t}^{c}\right)\right|<\varepsilon .
\end{aligned}
$$

We define an arc-length representation $z_{s}(0 \leq s \leq|A|)$ of $A$ so that $z_{0}=\zeta_{0}$. Let $A_{s}=\left\{z_{x} ; 0 \leq x \leq s\right\}(0 \leq s \leq|A|)$ and

$$
J=\left\{0 \leq s \leq|A| ; z_{s} \in A \cap B \text { or } z_{s} \notin \partial \Omega_{A_{s} \cup B_{t}}\right\} .
$$

We define a function on $[0,|A|]$ by

$$
D_{0}^{*} c\left(z_{s}, \zeta_{t}^{*}, \infty ; \Omega_{A_{s} \cup B_{\ell}}\right)= \begin{cases}D^{2} c\left(z_{s}, \zeta_{t}^{*}, \infty ; \Omega_{A_{s} \cup B_{t}}\right) & (s \in[0,|A|]-J) \\ 0 & (s \in J) .\end{cases}
$$

Since

$$
\frac{\partial}{\partial s} D c\left(\zeta_{t}^{*}, \infty ; \Omega_{A_{s} \cup B_{t}}\right)=0 \quad\left(z_{s} \notin \partial \Omega_{A_{s} \cup B_{t}}\right)
$$

we have

$$
D c\left(\zeta_{t}^{*}, \infty ; \Omega_{A \cup B_{t}}\right)=D c\left(\zeta_{t}^{*}, \infty ; B_{t}^{c}\right)+\frac{1}{4} \int_{0}^{|A|} D_{0}^{2} c\left(z_{s}, \zeta_{t}^{*}, \infty ; \Omega_{A_{s} \cup B_{t}}\right) d s .
$$

Since $\Omega_{A_{s} \cup B_{t}}$ is simply-connected, Proposition 20 shows that

$D_{0}^{2} c\left(z_{s}, \zeta_{t}^{*}, \infty ; \Omega_{A_{s} \cup B_{t}}\right) \leq 0$, and hence $D c\left(\zeta_{t}^{*}, \infty ; \Omega_{A \cup B_{t}}\right) \leq D c\left(\zeta_{t}^{*}, \infty ; B_{t}^{c}\right)$. Thus

$$
D c\left(\zeta_{t}, \infty ; \Omega_{A \cup B_{t}}\right) \leq D c\left(\zeta_{t}, \infty ; B_{t}^{c}\right)+2 \varepsilon .
$$

Since $\varepsilon>0$ is arbitrary, we obtain (51). Inequality (51) shows that

$$
\gamma(A \cup B) \leq \gamma(A)+\frac{1}{4} \int_{0}^{|B|} D c\left(\zeta_{t}, \infty ; B_{t}^{c}\right) d t=\gamma(A)+\gamma(B) .
$$

This completes the proof.

Here is another expression of $D^{2} c$ from the point of view of Löwner's D. E. for simply-connected domains [33, p. 387].

Proposition 22. Let $\Gamma$ be an arc in $\mathscr{A}$ with the arc-length representation $w_{t}(0 \leq t \leq|\Gamma|)$ and let $p_{t}$ be the conformal mapping from $E_{t}^{c}$ onto $\overline{\mathbf{D}}^{c}$ such that $p_{t}(\infty)=\infty$ and $p_{t}\left(w_{t}\right)=1$. Then

$$
D^{2} c\left(w_{t}, z, \infty ; \Gamma_{t}^{c}\right)=-\frac{4}{\gamma\left(\Gamma_{t}\right)} \frac{\partial \gamma}{\partial t}\left(\Gamma_{t}\right) \lambda_{t}(z) D c\left(z, \infty ; \Gamma_{t}^{c}\right)(0<t \leq|\Gamma|),
$$


where $\lambda_{t}(z)=\left\{\operatorname{Im} p_{t}(z)\right\}^{2} /\left|1-p_{t}(z)\right|^{4}$.

Corollary 23. Let $\Gamma$ and $\lambda_{t}(0 \leq t \leq|\Gamma|)$ be the same as above. Then

$$
\gamma(\Gamma)=\frac{1}{4} \int_{0}^{|\Gamma|} \exp \left\{-\int_{0}^{t} \frac{1}{\gamma\left(\Gamma_{s}\right)} \frac{\partial \gamma}{\partial s}\left(\Gamma_{s}\right) \lambda_{s}\left(w_{t}\right) d s\right\} d t
$$

Since $D c\left(z, \infty ; \Gamma_{t}^{c}\right) \geq 0$, (52) also shows that $D^{2} c\left(w_{t}, z, \infty ; \Gamma_{t}^{c}\right) \leq 0$. Let $q_{t}^{\dagger}(z)=q\left(z ; \Gamma_{t}^{c}\right)(0 \leq t \leq|\Gamma|)$. Then $q_{t}^{\dagger \prime}(\infty)=1 / \gamma\left(\Gamma_{t}\right)$. Since

$$
\begin{aligned}
-\frac{1}{i} \gamma\left(\Gamma_{t}\right) \frac{q_{t}^{\dagger}(z)}{q_{t}^{\dagger}(z)} d z & =-\frac{1}{i} \gamma\left(\Gamma_{t}\right) d \log q_{t}^{\dagger}(z) \\
& =-\gamma\left(\Gamma_{t}\right) d \arg q_{t}^{\dagger}(z) \geq 0 \text { on } \partial \Gamma_{t}^{c},
\end{aligned}
$$

we have

(54) $g\left(z ; \Gamma_{t}^{c}\right)=-\sqrt{\gamma\left(\Gamma_{t}\right) q_{t}^{\dagger}(z)} / q_{t}^{\dagger}(z), \quad \phi\left(z ; \Gamma_{t}^{c}\right)=\sqrt{\gamma\left(\Gamma_{t}\right) q_{t}^{\dagger}(z)}$, where a branch of $\sqrt{ }$ is chosen so that $\sqrt{q_{t}^{\dagger \prime}(\infty)}=1 / \sqrt{\gamma\left(\Gamma_{t}\right)}$. Thus

(55) $D c\left(z, \infty ; \Gamma_{t}^{c}\right)=\gamma\left(\Gamma_{t}\right)\left|q_{t}^{\dagger \prime}(z)\right|\left\{1-\left|q_{t}^{\dagger}(z)\right|^{-2}\right\}$.

Choosing a suitable parametric representation of Löwner's D. E., we obtain

$$
\text { (56) } \frac{\partial q_{t}^{\dagger}}{\partial t}(z)=\frac{1}{\gamma\left(\Gamma_{t}\right)} \frac{\partial \gamma}{\partial t}\left(\Gamma_{t}\right) q_{t}^{\dagger}(z) \frac{1+\kappa_{t}^{\dagger} q_{t}^{\dagger}(z)}{1-\kappa_{t}^{\dagger} q_{t}^{\dagger}(z)} \quad\left(\kappa_{t}^{\dagger}=\overline{\left.q_{t}^{\dagger}\left(w_{t}\right)\right)}\right. \text {. }
$$

In fact, for a pair $t<u$, there exists a conformal mapping $\Phi_{t, u}$ from $\overline{\mathbf{D}}^{c}$ onto a subdomain of $\overline{\mathbf{D}}^{c}$ such that $\Phi_{t, u}(\infty)=\infty, \Phi_{t, u}^{\prime}(\infty)>0$ and $q_{t}^{\dagger}=$ $\Phi_{t, u} \circ q_{u}^{\dagger}$. Poisson's formula shows that

$$
\log \frac{q_{t}^{\dagger}}{q_{u}^{\dagger}}=\log \frac{\Phi_{t, u}\left(q_{u}^{\dagger}\right)}{q_{u}^{\dagger}}=-\frac{1}{2 \pi} \int_{\psi t, u}^{\psi_{t, u}} \log \left|\Phi_{t, u}\left(e^{i \psi}\right)\right| \frac{e^{i \psi}+q_{u}^{\dagger}}{e^{i \psi}-q_{u}^{\dagger}} d \psi,
$$

where $\left(\psi_{t, u}, \psi_{t, u}^{\prime}\right)=\left\{0 \leq \psi<2 \pi ;\left|\Phi_{t, u}\left(e^{i \psi}\right)\right|>1\right\}$. Analogously,

$$
\log \frac{\gamma\left(\Gamma_{u}\right)}{\gamma\left(\Gamma_{t}\right)}=\log \frac{q_{t}^{\dagger \prime}(\infty)}{q_{u}^{\dagger \prime}(\infty)}=\frac{1}{2 \pi} \int_{\psi_{t, u}}^{\psi_{t}^{\prime}, u} \log \left|\Phi_{t, u}\left(e^{i \psi}\right)\right| d \psi .
$$

Note that $\lim _{u \downarrow t} \psi_{t, u}=\lim _{u \downarrow t} \psi_{t, u}^{\prime}=q_{t}^{\dagger}\left(w_{t}\right)$. Letting $u$ tend to $t$, we obtain (56). Equality (55) shows that

$$
\begin{aligned}
\frac{\partial}{\partial t} D c\left(\cdot, \infty ; \Gamma_{t}^{c}\right)= & \frac{\partial \gamma}{\partial t}\left(\Gamma_{t}\right)\left|q_{t}^{\dagger}\right|\left\{1-\left|q_{t}^{\dagger}\right|^{-2}\right\} \\
& +\gamma\left(\Gamma_{t}\right) \frac{\partial\left|q_{t}^{\dagger \prime}\right|}{\partial t}\left\{1-\left|q_{t}^{\dagger}\right|^{-2}\right\}+2 \gamma\left(\Gamma_{t}\right)\left|q_{t}^{\dagger \prime}\right|\left|q_{t}^{\dagger}\right|^{-3} \frac{\partial\left|q_{t}^{\dagger}\right|}{\partial t} .
\end{aligned}
$$


Equality (56) yields that

$$
\begin{aligned}
\frac{\partial\left|q_{t}^{\dagger}\right|}{\partial t} & =\frac{1}{\gamma\left(\Gamma_{t}\right)} \frac{\partial \gamma}{\partial t}\left(\Gamma_{t}\right)\left|q_{t}^{\dagger}\right| \frac{1-\left|q_{t}^{\dagger}\right|^{2}}{\left|1-\kappa_{t}^{\dagger} q_{t}^{\dagger}\right|^{2}}, \\
\frac{\partial\left|q_{t}^{\dagger \prime}\right|}{\partial t} & =\frac{1}{\gamma\left(\Gamma_{t}\right)} \frac{\partial \gamma}{\partial t}\left(\Gamma_{t}\right)\left|q_{t}^{\dagger}\right| \operatorname{Re} \frac{1+2 \kappa_{t}^{\dagger} q_{t}^{\dagger}-\kappa_{t}^{\dagger 2} q_{t}^{\dagger 2}}{\left(1-\kappa_{t}^{\dagger} q_{t}^{\dagger}\right)^{2}}
\end{aligned}
$$

and hence

$$
\begin{array}{rl}
\frac{\partial}{\partial t} D & c\left(\cdot, \infty ; \Gamma_{t}^{c}\right)=\frac{\partial \gamma}{\partial t}\left(\Gamma_{t}\right)\left|q_{t}^{\dagger}\right|\left\{1-\left|q_{t}^{\dagger}\right|^{-2}\right\} \\
& +\frac{\partial \gamma}{\partial t}\left(\Gamma_{t}\right)\left|q_{t}^{\dagger}\right|\left\{1-\left|q_{t}^{\dagger}\right|^{-2}\right\} \operatorname{Re} \frac{1+2 \kappa_{t}^{\dagger} q_{t}^{\dagger}-\kappa_{t}^{\dagger 2} q_{t}^{\dagger 2}}{\left(1-\kappa_{t}^{\dagger} q_{t}^{\dagger}\right)^{2}} \\
& +2 \frac{\partial \gamma}{\partial t}\left(\Gamma_{t}\right)\left|q_{t}^{\dagger}\right|\left|q_{t}^{\dagger}\right|^{-2} \frac{1-\left|q_{t}^{\dagger}\right|^{2}}{\left|1-\kappa_{t}^{\dagger} q_{t}^{\dagger}\right|^{2}} \\
= & \frac{\partial \gamma}{\partial t}\left(\Gamma_{t}\right)\left|q_{t}^{\dagger}\right|\left\{1-\left|q_{t}^{\dagger}\right|^{-2}\right\}\left(1+\operatorname{Re} \frac{1+2 \kappa_{t}^{\dagger} q_{t}^{\dagger}-\kappa_{t}^{\dagger 2} q_{t}^{\dagger 2}}{\left(1-\kappa_{t}^{\dagger} q_{t}^{\dagger}\right)^{2}}-\frac{2}{\left|1-\kappa_{t}^{\dagger} q_{t}^{\dagger}\right|^{2}}\right) \\
= & \frac{2}{\gamma\left(\Gamma_{t}\right)} \frac{\partial \gamma}{\partial t}\left(I_{t}\right) D c\left(\cdot, \infty ; \Gamma_{t}^{c}\right)\left(\operatorname{Re} \frac{1}{\left(1-\kappa_{t}^{\dagger} q_{t}^{\dagger}\right)^{2}}-\frac{1}{\left|1-\kappa_{t}^{\dagger} q_{t}^{\dagger}\right|^{2}}\right) \\
= & -\frac{4}{\gamma\left(\Gamma_{t}\right)} \frac{\partial \gamma}{\partial t}\left(\Gamma_{t}\right) D c\left(\cdot, \infty ; \Gamma_{t}^{c}\right) \frac{\left(\operatorname{Im} \kappa_{t}^{\dagger} q_{t}^{\dagger}\right)^{2}}{\left|1-\kappa_{t}^{\dagger} q_{t}^{\dagger}\right|^{4}} \cdot
\end{array}
$$

Since $p_{t}=\kappa_{t}^{\dagger} q_{t}^{\dagger}$ and $\partial D c\left(z, \infty ; \Gamma_{t}^{c}\right) / \partial t=D^{2} c\left(w_{t}, z, \infty ; \Gamma_{t}^{c}\right) / 4$, we obtain (52). Combined with Theorem 1, (52) immediately yields (53).

It is interesting to compare our variational formula with Löwner's D. E.. We here point out that (56) is deduced from Theorem 8. Recall (54). Since $q_{t}^{\dagger}(z)^{-1}=-g\left(z ; \Gamma_{t}^{c}\right) \phi\left(z ; \Gamma_{t}^{c}\right)^{-1}$, Theorem 8 shows that

$$
\begin{aligned}
& q_{t}^{\dagger}(z)^{-2} \frac{\partial q_{t}^{\dagger}}{\partial t}(z)=\left\{\frac{\partial g}{\partial t}\left(z ; \Gamma_{t}^{c}\right)+q_{t}^{\dagger}(z)^{-1} \frac{\partial \phi}{\partial t}\left(z ; \Gamma_{t}^{c}\right)\right\} \phi\left(z ; \Gamma_{t}^{c}\right)^{-1} \\
& \quad=-\frac{1}{4}\left\{D K\left(w_{t}, z, \infty ; \Gamma_{t}^{c}\right)+q_{t}^{\dagger}(z)^{-1} D L\left(w_{t}, z, \infty ; \Gamma_{t}^{c}\right)\right\} \phi\left(z ; \Gamma_{t}^{c}\right)^{-1}
\end{aligned}
$$

Since

$$
\begin{aligned}
& K\left(w, \bar{z} ; \Gamma_{t}^{c}\right)=-\frac{1}{1-q_{t}^{\dagger}(w) q_{t}^{\dagger}(\boldsymbol{z})} \sqrt{q_{t}^{\dagger \prime}(w)} \sqrt{\overline{q_{t}^{\dagger}(\boldsymbol{z})}} \\
& L\left(w, z ; \Gamma_{t}^{c}\right)=\frac{1}{q_{t}^{\dagger}(w)-q_{t}^{\dagger}(\boldsymbol{z})} \sqrt{q_{t}^{\dagger \prime}(w)} \sqrt{q_{t}^{\dagger \prime}(\boldsymbol{z})}
\end{aligned}
$$

we have 


$$
\begin{aligned}
& -\frac{1}{4}\left\{D K\left(w, z, \infty ; \Gamma_{t}^{c}\right)+q_{t}^{\dagger}(z)^{-1} D L\left(w, z, \infty ; \Gamma_{t}^{c}\right)\right\} \phi\left(z ; \Gamma_{t}^{c}\right)^{-1} \\
& =\frac{1}{4}\left(L\left(w, z ; \Gamma_{t}^{c}\right) \overline{\phi\left(w ; \Gamma_{t}^{c}\right)}-\overline{K\left(w, \bar{z} ; \Gamma_{t}^{c}\right)} g\left(w ; \Gamma_{t}^{c}\right)\right. \\
& \left.+q_{t}^{\dagger}(z)^{-1}\left\{L\left(w, z ; \Gamma_{t}^{c}\right) \overline{g\left(w ; \Gamma_{t}^{c}\right)}-\bar{K} \overline{\left(w, \bar{z} ; \Gamma_{t}^{c}\right)} \phi\left(w ; \Gamma_{t}^{c}\right)\right\}\right) \phi\left(z ; \Gamma_{t}^{c}\right)^{-1} \\
& =\frac{1}{4}\left|q_{t}^{\dagger}(w)\right|\left(\frac{1}{q_{t}^{\dagger}(w)-q_{t}^{\dagger}(z)}-\frac{1}{q_{t}^{\dagger}(w)\left\{1-\overline{q_{t}^{\dagger}(w)} q_{t}^{\dagger}(z)\right\}}\right. \\
& \left.-\frac{1}{\overline{q_{t}^{\dagger}(w)} q_{t}^{\dagger}(z)\left\{q_{t}^{\dagger}(w)-q_{t}^{\dagger}(z)\right\}}+\frac{1}{q_{t}^{\dagger}(z)\left\{1-\overline{q_{t}^{\dagger}(w)} q_{t}^{\dagger}(z)\right\}}\right) \\
& =\frac{1}{4}\left|q_{t}^{\dagger}(w)\right|\left(\frac{-1+\overline{q_{t}^{\dagger}(w)} q_{t}^{\dagger}(z)}{\overline{q_{t}^{\dagger}(w)} q_{t}^{\dagger}(z)\left\{q_{t}^{\dagger}(w)-q_{t}^{\dagger}(z)\right\}}+\frac{q_{t}^{\dagger}(w)-q_{t}^{\dagger}(z)}{q_{t}^{\dagger}(w) q_{t}^{\dagger}(z)\left\{1-\overline{q_{t}^{\dagger}(w)} q_{t}^{\dagger}(z)\right\}}\right) \\
& =-\frac{\left|q_{t}^{\dagger \prime}(w)\right|}{4\left|q_{t}^{\dagger}(w)\right|^{2} q_{t}^{\dagger}(z)\left\{q_{t}^{\dagger}(w)-q_{t}^{\dagger}(z)\right\}\left\{1-\overline{q_{t}^{\dagger}(w)} q_{t}^{\dagger}(z)\right\}} \\
& \times\left(-q_{t}^{\dagger}(w)\left\{1-\overline{q_{t}^{\dagger}(w)} q_{t}^{\dagger}(z)\right\}^{2}+\overline{q_{t}^{\dagger}(w)}\left\{q_{t}^{\dagger}(w)-q_{t}^{\dagger}(z)\right\}^{2}\right) \\
& =\frac{\left|q_{t}^{\dagger}(w)\right|\left\{\left|q_{t}^{\dagger}(w)\right|^{2}-1\right\}\left\{q_{t}^{\dagger}(w)^{2}-\left|q_{t}^{\dagger}(w)\right|^{2} q_{t}^{\dagger}(z)^{2}\right\}}{4 q_{t}^{\dagger}(w)\left|q_{t}^{\dagger}(w)\right|^{2} q_{t}^{\dagger}(z)\left\{q_{t}^{\dagger}(w)-q_{t}^{\dagger}(z)\right\}\left\{1-\overline{\left.q_{t}^{\dagger}(w) q_{t}^{\dagger}(z)\right\}}\right.} .
\end{aligned}
$$

Put $w=w_{u}(0<t<u)$. Letting $u$ tend to $t$, we have

$$
\frac{\partial q_{t}^{\dagger}}{\partial t}(z)=\gamma_{t}^{\dagger} q_{t}^{\dagger}(z) \frac{1+\overline{q_{t}^{\dagger}\left(w_{t}\right)} q_{t}^{\dagger}(z)}{1-\overline{q_{t}^{\dagger}\left(w_{t}\right)} q_{t}^{\dagger}(z)}
$$

with $\gamma_{t}^{\dagger}=\lim _{u ! t}\left|q_{t}^{\dagger \prime}\left(w_{u}\right)\right|\left\{\left|q_{t}^{\dagger}\left(w_{u}\right)\right|^{2}-1\right\} / 4$. Since $q_{t}^{\dagger \prime}(\infty)=1 / \gamma\left(\Gamma_{t}\right)$, this equality shows that

$$
-\gamma\left(\Gamma_{t}\right)^{-2} \frac{\partial \gamma}{\partial t}\left(\Gamma_{t}\right)=-\gamma_{t}^{\dagger} \gamma\left(\Gamma_{t}\right)^{-1}, \text { i.e., } \gamma_{t}^{\dagger}=\gamma\left(\Gamma_{t}\right)^{-1} \frac{\partial \gamma}{\partial t}\left(\Gamma_{t}\right)
$$

Thus we obtain (56).

\section{§9. Application to doubly-connected domains}

In this section, we study $D^{2} c(w, z, \zeta ; \Omega)$ for doubly-connected domains $\Omega$. We begin by showing some examples of the computation of $D^{2} c$. Let $R_{\rho}=\{\rho<|z|<1\}(0<\rho<1)$. Then

$$
\begin{aligned}
& K\left(z, \bar{\zeta} ; R_{\rho}\right)=\sum_{n=-\infty}^{\infty} \frac{(z \bar{\zeta})^{n}}{1+\rho^{2 n+1}}, \\
& L\left(z, \zeta ; R_{\rho}\right)=\frac{1}{z-\zeta}+\sum_{n=0}^{\infty} \frac{\rho^{2 n+1}\left(z^{2 n+1}-\zeta^{2 n+1}\right)}{(z \zeta)^{n+1}\left(1+\rho^{2 n+1}\right)} \quad[21, \text { p. 391]}
\end{aligned}
$$

Thus, in the case of $R_{\rho}$, we can write $D^{2} c$, explicitly. Using Jacobian 
elliptic functions [12], we can rewrite $D^{2} c$ as follows. Define a modulus $0<k<1$ of elliptic functions by $\rho=e^{-\pi K^{\prime} / K}$, where $K=K(k)$ is the complete elliptic integral of the first kind and $K^{\prime}=K\left(\sqrt{1-k^{2}}\right)$. Let $s n$ and $d n$ denote the Jacobian elliptic functions with modulus $k$. Thus

$$
\begin{aligned}
& D^{2} c\left(w, z, \zeta ; R_{\rho}\right)=\frac{2 K^{3}}{\pi^{3}|w z \zeta|} \\
& \quad \times \operatorname{Im}\left[\left(\frac{d n(\xi-u)}{s n(\xi-u)} \frac{d n(\bar{\xi}-v)}{s n(\bar{\xi}-v)}-\frac{d n(\bar{\xi}-u)}{s n(\bar{\xi}-u)} \frac{d n(\xi-v)}{s n(\xi-v)}\right) \frac{d n(\bar{u}-\bar{v})}{s n(\bar{u}-\bar{v})}\right. \\
& \left.-\left(\frac{d n(\xi-u)}{s n(\xi-u)} \frac{d n(\bar{\xi}-\bar{v})}{s n(\bar{\xi}-\bar{v})}-\frac{d n(\bar{\xi}-u)}{s n(\bar{\xi}-u)} \frac{d n(\xi-\bar{v})}{s n(\xi-\bar{v})}\right) \frac{d n(\bar{u}-v)}{s n(\bar{u}-v)}\right], \\
& \xi=\frac{K}{i \pi} \log w, \quad u=\frac{K}{i \pi} \log z, \quad v=\frac{K}{i \pi} \log \zeta,
\end{aligned}
$$

where a branch of $\log$ is chosen so that $\log x \geq 0(x \geq 1)$. In fact, put

$$
\begin{aligned}
K^{*}(z, \bar{\zeta}) & =-\frac{K}{i \pi} \frac{d n(u-\bar{v})}{\operatorname{sn}(u-\bar{v})} \frac{1}{\sqrt{z} \sqrt{\bar{\zeta}}}, \\
L^{*}(z, \zeta) & =\frac{K}{i \pi} \frac{d n(u-v)}{\operatorname{sn}(u-v)} \frac{1}{\sqrt{z} \sqrt{\zeta}} .
\end{aligned}
$$

Since $s n(u+2 K)=-s n u, d n(u+2 K)=d n u$ and $\sqrt{e^{2 \pi i}}=-1$, these functions are single-valued in $R_{\rho}$ as functions of $z$. Since $s n u=u+o(u)$, $d n u=1+o(u)(u \rightarrow 0)$, we have $L^{*}(z, \zeta)=1 /(z-\zeta)+O(1)(z \rightarrow \zeta)$. If $|z|=1$, then $u$ is real-valued, and hence $L^{*}(z, \zeta) \sqrt{z}=\overline{K^{*}(z, \bar{\zeta}) \sqrt{ } \bar{z}}$. Since $(1 / i)(|z| / z) d z=|d z|$ on $|z|=1$, we have $(1 / i) L^{*}(z, \zeta) d z=\overline{K^{*}(z, \zeta) \mid}|d z|$ on $|z|$ $=1$. If $|z|=\rho$, then $u=i K^{\prime}+(K / \pi) \arg z$. Since $\operatorname{sn}\left(u+i K^{\prime}\right)=\operatorname{sn}\left(u-i K^{\prime}\right)$, $d n\left(u+i K^{\prime}\right)=-d n\left(u-i K^{\prime}\right)$, we have $L^{*}(z, \zeta) \sqrt{z}=-\overline{K^{*}(z, \bar{\zeta}) \sqrt{z}}$ on $|z|=\rho$. Since $(1 / i)(|z| \mid z) d z=-|d z|$ on $|z|=\rho$, we have $(1 / i) L^{*}(z, \zeta) d z=$ $\overline{K^{*}(z, \bar{\zeta})}|d z|$ on $|z|=\rho$. Thus $K^{*}(z, \bar{\zeta})=K\left(z, \bar{\zeta} ; R_{\rho}\right)$ and $L^{*}(z, \zeta)=L\left(z, \zeta ; R_{\rho}\right)$, which gives (57). Let $\Omega_{\rho}$ be a radial slit domain which is conformally equivalent to $R_{\rho}$. Then

$$
\begin{aligned}
Q_{\rho, z, \zeta}(w) & =\exp \left\{\mathscr{N}_{\rho}(w, \zeta)-\mathscr{N}_{\rho}(w, z)\right\} \\
& =\frac{w-z}{w-\zeta} \exp \left(-\sum_{n=1}^{\infty} \frac{X_{n}(w)+Y_{n}(w) \rho^{2 n}}{n\left(1-\rho^{2 n}\right)}\right) \\
X_{n}(w) & =(\bar{z} w)^{n}-(\bar{\zeta} w)^{n}, \\
Y_{n}(w) & =(w / z)^{n}-(w / \zeta)^{n}-(z / w)^{n}+(\zeta / w)^{n}
\end{aligned}
$$

is a conformal mapping from $R_{\rho}$ onto $\Omega_{\rho}$ such that $Q_{\rho, z, \xi}(z)=0$ and $Q_{\rho, z, \xi}(\zeta)=\infty$, where $\mathscr{N}_{\rho}(w, \xi)$ is a multi-valued analytic function in 
$R_{\rho}-\{\xi\}$ whose real part is the Neumann function with pole at $\xi$ [21, p. 377]. Hence, in the case of $\Omega_{\rho}$ also, we can write $D^{2} c$ explicitly. Here is an example of $D^{2} c$ which is expressed by elementary functions.

Proposition 24. Let $\Omega=\left\{\bigcup_{k=1}^{n}\left[a_{k}, b_{k}\right]\right\}^{c}\left(a_{1}<b_{1}<\cdots<a_{n}<b_{n}\right)$. Then

$$
\begin{aligned}
& D^{2} c(w, z, \zeta ; \Omega)=\frac{1}{4|M(w) M(z) M(\zeta)|} \\
& \times \operatorname{Re}\left[\left(\frac{M(w)+\overline{M(z)}}{\bar{w}-z} \frac{\overline{M(w)}-M(\zeta)}{\bar{w}-\zeta}\right.\right. \\
& \left.\quad-\frac{\overline{M(w)}-M(z)}{\bar{w}-z} \frac{M(w)+M(\zeta)}{w-\zeta}\right) \frac{\overline{M(z)}+\overline{M(\zeta)}}{\bar{z}-\bar{\zeta}} \\
& -\left(\frac{M(w)+M(z)}{w-z} \frac{\overline{M(w)}+\overline{M(\zeta)}}{\bar{w}-\bar{\zeta}}\right. \\
& \left.\left.\quad-\frac{\overline{M(w)}-M(z)}{\bar{w}-z} \frac{M(w)-\overline{M(\zeta)}}{w-\bar{\zeta}}\right) \frac{\overline{M(z)}-M(\zeta)}{\bar{z}-\zeta}\right], \\
& M(\xi)=\prod_{k=1}^{n} \sqrt{\left(b_{k}-\xi\right) /\left(a_{k}-\xi\right)} \quad(\xi=w, z, \zeta) .
\end{aligned}
$$

Note that $\sqrt{M(z)}$ is single-valued. Since

$$
\begin{aligned}
K(z, \bar{\zeta} ; \Omega) & =\frac{1}{z-\bar{\zeta}} \sinh \left(h_{z}-\bar{h}_{\zeta}\right), \quad L(z, \zeta ; \Omega)=\frac{1}{z-\zeta} \cosh \left(h_{z}-h_{\zeta}\right), \\
h_{\xi} & =\frac{1}{4} \sum_{k=1}^{n} \int_{a_{k}}^{b_{k}} \frac{1}{x-\xi} d x \quad(\xi=z, \zeta) \quad([3],[20]),
\end{aligned}
$$

we have

$$
\begin{aligned}
D^{2} c(w, & z, \zeta ; \Omega) \\
= & 2 \operatorname{Re}\left[\frac{1}{(w-z)(\bar{w}-\zeta)(\bar{z}-\bar{\zeta})} \cosh \left(h_{w}-h_{z}\right) \sinh \left(\bar{h}_{w}-h_{\zeta}\right) \cosh \left(\bar{h}_{z}-\bar{h}_{\zeta}\right)\right. \\
& -\frac{1}{(\bar{w}-z)(w-\zeta)(\bar{z}-\bar{\zeta})} \sinh \left(\bar{h}_{w}-h_{z}\right) \cosh \left(h_{w}-h_{\zeta}\right) \cosh \left(\bar{h}_{z}-\bar{h}_{\zeta}\right) \\
& -\frac{1}{(w-z)(\bar{w}-\bar{\zeta})(\bar{z}-\zeta)} \cosh \left(h_{w}-h_{z}\right) \cosh \left(\bar{h}_{w}-\bar{h}_{\zeta}\right) \sinh \left(\bar{h}_{z}-h_{\zeta}\right) \\
& \left.+\frac{1}{(\bar{w}-z)(w-\bar{\zeta})(\bar{z}-\zeta)} \sinh \left(\bar{h}_{w}-h_{z}\right) \sinh \left(h_{w}-\bar{h}_{\zeta}\right) \sinh \left(\bar{h}_{z}-h_{\zeta}\right)\right] \\
= & \frac{1}{4} \operatorname{Re}\left[\frac{1}{(w-z)(\bar{w}-\zeta)(\bar{z}-\bar{\zeta})}\right. \\
& \times\left(\frac{\sqrt{M(w)}}{\sqrt{M(z)}}+\frac{\sqrt{M(z)}}{\sqrt{M(w)}}\right)\left(\frac{(\sqrt{\overline{M(w)})}}{\sqrt{M(\zeta)}}-\frac{\sqrt{M(\zeta)}}{(\sqrt{\bar{M}(w)})}\right)\left(\frac{(\sqrt{\overline{M(z)}})}{(\overline{\sqrt{M(\zeta)})}}+\frac{(\sqrt{\overline{M(\zeta)})}}{(\sqrt{\overline{M(z)})})}\right.
\end{aligned}
$$




$$
\begin{aligned}
& -\frac{1}{(\bar{w}-z)(w-\zeta)(\bar{z}-\bar{\zeta})} \\
& \times\left(\frac{(\sqrt{\overline{M(w)})}}{\sqrt{\overline{M(z)}}}-\frac{\sqrt{M(z)}}{(\overline{\sqrt{M(w)})}}\right)\left(\frac{\sqrt{M(w)}}{\sqrt{M(\zeta)}}+\frac{\sqrt{M(z)}}{\sqrt{M(w)}}\right)\left(\frac{(\sqrt{\overline{M(z)}})}{(\overline{\sqrt{M(\zeta)})}}+\frac{(\sqrt{\bar{M}(\zeta)})}{(\overline{\sqrt{M(z)})}}\right) \\
& -\frac{1}{(w-z)(\bar{w}-\bar{\zeta})(\bar{z}-\zeta)} \\
& \times\left(\frac{\sqrt{M(w)}}{\sqrt{M(z)}}+\frac{\sqrt{M(z)}}{\sqrt{M(w)}}\right)\left(\frac{(\sqrt{\bar{M}(w)})}{\overline{(\sqrt{M(\zeta)})}}+\frac{(\sqrt{\overline{M(\zeta)})}}{(\sqrt{M(w)})}\right)\left(\frac{(\sqrt{M(z)})}{\sqrt{M(\zeta)}}-\frac{\sqrt{M(\zeta)}}{(\sqrt{M(z))}}\right) \\
& +\frac{1}{(\bar{w}-z)(w-\bar{\zeta})(\bar{z}-\zeta)} \\
& \left.\times\left(\frac{(\sqrt{M(w)})}{\sqrt{M(z)}}-\frac{\sqrt{M(z)}}{(\sqrt{M(w)})}\right)\left(\frac{\sqrt{M(w)}}{\overline{(\sqrt{M(\zeta))}}}-\frac{\overline{(\sqrt{M(\zeta)})}}{\sqrt{M(w)}}\right)\left(\frac{(\sqrt{\overline{M(z)})}}{\sqrt{M(\zeta)}}-\frac{\sqrt{M(\zeta)}}{(\sqrt{M(z)})}\right)\right] .
\end{aligned}
$$

which yields (58).

Note that any triply-connected domain is conformally equivalent to a radial slit ring [33, p. 413] which is equivalent to a domain of form in this proposition. Thus (58) is applicable to simply, doubly, and triply connected domains.

There remains to prove Theorem 3 in the case where $\Omega$ is doublyconnected. There are two methods of the proof of this theorem; a geometric method by (58) and an analytic method by theta functions [12] and (57). In this note, we use the first method. We shall see that the required inequality is deduced from an inequality of Möbius type in elementary geometry. It is sufficient to show that

(59) $\quad D^{2} c\left(x, i s,-i t ; E^{c}\right) \leq 0$

for all $s>0, t>0, x \in \mathbf{R}-E$ and closed sets $E$ of form:

$$
\begin{aligned}
& E=[a, b] \cup\left[a^{\prime}, b^{\prime}\right] \quad\left(a<b<a^{\prime}<b^{\prime}\right), \\
& E=\left(-\infty, b^{\prime}\right] \cup[a, b] \cup\left[a^{\prime}, \infty\right] \quad\left(b^{\prime}<a<b<a^{\prime}\right) .
\end{aligned}
$$

In fact, once (59) has been shown, the required inequality is deduced as follows. By Proposition 2, we may assume that $\Omega$ is a ring $R_{\rho}$. Since $D^{2} c\left(w, z, \zeta ; R_{\rho}\right)$ is continuous in $R_{\rho} \times R_{\rho} \times R_{\rho}-\{w \neq z, w \neq \zeta, z \neq \zeta\}$, we may assume that any straight line passing through 0 contains at most one point in $\{w, z, \zeta\}$. Rotating the coordinate axes if necessary, we may assume that $w \in \mathbf{R}$. We may also assume that $\operatorname{Im} z>0$ and $\operatorname{Im} \zeta<0$; this 
is possible because $D^{2} c\left(w, z, \zeta ; R_{\rho}\right)$ is invariant for any permutation of a triple $(w, z, \zeta)$. Using elliptic functions, we can define a conformal mapping $f$ from $R_{\rho}$ onto a domain $F^{c}$ of form $F=\left[a^{\prime \prime}, b^{\prime \prime}\right] \cup\left[a^{*}, b^{*}\right] \subset \mathbf{R}$ so that $f(w) \in \mathbf{R}, \operatorname{Im} f(z)>0$ and $\operatorname{Im} f(\zeta)<0$. Take a linear transformation $g$ such that $g \circ f(z) / i>0, g \circ f(\zeta) / i<0$ and $g(\mathbf{R} \cup\{\infty\})=\mathbf{R} \cup\{\infty\}$. Then $g(F)$ is a union of two continua on $\mathbf{R} \cup\{\infty\}$. Thus we obtain a conformal mapping $g \circ f$ from $R_{\rho}$ onto $E^{c}=g(F)^{c}$ of type (59). We have $g \circ f(w) \in \mathbf{R} \cup\{\infty\}$. Choosing a point in $R_{\rho}$ sufficiently near to $w$ if necessary, we may assume that $g \circ f(w) \neq \infty$. Applying (59) to $x=g \circ f(w), s=g \circ f(z) / i$ and $t=$ $-g \circ f(\zeta) / i$, we obtain $D^{2} c\left(w, z, \zeta ; R_{\rho}\right) \leq 0$, by Proposition 2 .

To prove (59), we assume, for a while, that $E=[a, b] \cup\left[a^{\prime}, b^{\prime}\right](a<$ $b<0<a^{\prime}<b^{\prime}$ ). Applying (58) to $\Omega=E^{c}$ and $w=x \in \mathbf{R}-E$, we have

$$
\begin{aligned}
D^{2} c\left(x, z, \zeta ; E^{c}\right)=\frac{1}{4|M(x) M(z) M(\zeta)|} \operatorname{Re}\left[\frac{\overline{M(z)}+\overline{M(\zeta)}}{(x-z)(x-\zeta)(\bar{z}-\bar{\zeta})}\right. \\
\quad \times\{(M(x)+M(z))(M(x)-M(\zeta))-(M(x)-M(z))(M(x)+M(\zeta))\} \\
\quad-\frac{\overline{M(z)}-M(\zeta)}{(x-z)(x-\bar{\zeta})(\bar{z}-\zeta)} \\
\quad \times\{(M(x)+M(z))(M(x)+\overline{M(\zeta)}-(M(x)-M(z))(M(x)-\overline{M(\zeta))\}}] \\
=\frac{1}{2|M(z) M(\zeta)||(x-z)(x-\zeta)|^{2}} \\
\quad \times \operatorname{Re}\left[\frac{(x-\bar{z})(x-\bar{\zeta}) \overline{(M(z)}+\overline{M(\zeta))}(M(z)-M(\zeta))}{\bar{z}-\bar{\zeta}}\right. \\
\quad-\frac{(x-\bar{z})(x-\zeta)}{\bar{z}-\zeta}(\overline{M(z)}-M(\zeta))(M(z)+\overline{M(\zeta))}] \\
=\frac{A x^{2}+B x+C}{2|M(z) M(\zeta)||(x-z)(x-\zeta)|^{2}}
\end{aligned}
$$

with

$$
\begin{array}{r}
A=\operatorname{Re}\left\{\frac{1}{\bar{z}-\bar{\zeta}}(\overline{M(z)}+\overline{M(\zeta)})(M(z)-M(\zeta))\right. \\
-\frac{1}{\bar{z}-\zeta}(\overline{M(z)}-M(\zeta))(M(z)+\overline{M(\zeta))}\}, \\
B=\operatorname{Re}\left\{-\frac{\bar{z}+\bar{\zeta}}{\bar{z}-\bar{\zeta}} \overline{(M(z)}+\overline{M(\zeta)}\right)(M(z)-M(\zeta)) \\
+\frac{\bar{z}+\zeta}{\bar{z}-\zeta}(\overline{M(z)}-M(\zeta))(M(z)+\overline{M(\zeta))}\},
\end{array}
$$




$$
\begin{aligned}
C=\operatorname{Re}\left\{\frac{\overline{z \zeta}}{\bar{z}-\zeta}\right. & (\overline{M(z)}+\overline{M(\zeta)})(M(z)-M(\zeta)) \\
& -\frac{\bar{z} \zeta}{\bar{z}-\zeta}(\overline{M(z)}-M(\zeta))(M(z)+\overline{M(\zeta))}\} .
\end{aligned}
$$

(Here we used $M(x)>0(x \in \mathbf{R}-E)$.) Since $A x^{2}+B x+C$ is quadratic, it is sufficient to show that

$$
B^{2}-4 A C<0, \quad A<0 .
$$

First we show $B^{2}-4 A C<0$. To express $B^{2}-4 A C$ in terms of geometry, we write

$$
r_{\xi} e^{i \theta_{\xi}}=M(\xi)^{2}=\frac{(b-\xi)\left(b^{\prime}-\xi\right)}{(a-\xi)\left(a^{\prime}-\xi\right)} \quad\left(r_{\xi}>0,\left|\theta_{\xi}\right|<\pi, \xi=z, \zeta\right) .
$$

Then, putting $z=i s, \zeta=-i t$, we have

$$
\begin{aligned}
A & =-2 \sqrt{r_{z} r_{\zeta}}\left\{\frac{1}{s+t} \sin \frac{\theta_{z}-\theta_{\zeta}}{2}+\frac{1}{s-t} \sin \frac{\theta_{z}+\theta_{\zeta}}{2}\right\} \\
B & =-\frac{s-t}{s+t}\left(r_{z}-r_{\zeta}\right)+\frac{s+t}{s-t}\left(r_{z}-r_{\zeta}\right) \\
& =\frac{4 s t}{(s+t)(s-t)} \sqrt{r_{z} r_{\zeta}}\left\{\sqrt{r_{z} / r_{\zeta}}-\sqrt{r_{\zeta} / r_{z}}\right\}, \\
C & =-2 \sqrt{r_{z} r_{\zeta}}\left\{\frac{s t}{s+t} \sin \frac{\theta_{z}-\theta_{\zeta}}{2}-\frac{s t}{s-t} \sin \frac{\theta_{z}+\theta_{\zeta}}{2}\right\},
\end{aligned}
$$

and hence

$$
\begin{aligned}
\frac{B^{2}-4}{16 r_{z}} r_{\zeta} & =\left\{\frac{s t}{(s+t)(s-t)}\right\}^{2}\left\{r_{z} r_{\zeta}^{-1}+r_{z}^{-1} r_{\zeta}-2\right\} \\
& -\left\{\frac{1}{s+t} \sin \frac{\theta_{z}-\theta_{\zeta}}{2}+\frac{1}{s-t} \sin \frac{\theta_{z}+\theta_{\zeta}}{2}\right\} \\
& \times\left\{\frac{s t}{s+t} \sin \frac{\theta_{z}-\theta_{\zeta}}{2}-\frac{s t}{s-t} \sin \frac{\theta_{z}+\theta_{\zeta}}{2}\right\} \\
= & \left\{\frac{s t}{(s+t)(s-t)}\right\}^{2}\left\{r_{z} r_{\zeta}^{-1}+r_{z}^{-1} r_{\zeta}-2\right\} \\
& -\frac{s t}{2(s+t)^{2}}\left\{1-\cos \left(\theta_{z}-\theta_{\zeta}\right)\right\}+\frac{s t}{2(s-t)^{2}}\left\{1-\cos \left(\theta_{z}+\theta_{\zeta}\right)\right\} \\
= & \left\{\frac{s t}{(s+t)(s-t)}\right\}^{2}\left\{r_{z} r_{\zeta}^{-1}+r_{z}^{-1} r_{\zeta}-2 \cos \theta_{z} \cos \theta_{\zeta}+\frac{s^{2}+t^{2}}{s t} \sin \theta_{z} \sin \theta_{\zeta}\right\} \\
= & \left\{\frac{s t}{(s+t)(s-t)}\right\}^{2} D, \quad \text { say. }
\end{aligned}
$$


Note that $0<\theta_{z}<\pi,-\pi<\theta_{\zeta}<0$. Let $R, Q, R^{\prime}, Q^{\prime}, r, q, r^{\prime}, q^{\prime}, \alpha, \alpha^{\prime}, \beta, \beta^{\prime}$ be the positive numbers in the figure in $\S 1$. Then

$$
\begin{aligned}
D= & \frac{Q Q^{\prime} r r^{\prime}}{R R^{\prime} q q^{\prime}}+\frac{R R^{\prime} q q^{\prime}}{Q Q^{\prime} r r^{\prime}}-2 \cos \left(\alpha+\alpha^{\prime}\right) \cos \left(\beta+\beta^{\prime}\right) \\
& -\frac{s^{2}+t^{2}}{s t} \sin \left(\alpha+\alpha^{\prime}\right) \sin \left(\beta+\beta^{\prime}\right) .
\end{aligned}
$$

A calculation yields that

$$
D=-\frac{1}{R Q R^{\prime} Q^{\prime} r q r^{\prime} q^{\prime}}(b-a)\left(b^{\prime}-a^{\prime}\right)\left(b^{\prime}-a\right)\left(a^{\prime}-b\right)\left(s^{2}-t^{2}\right)^{2}
$$

Thus $B^{2}-4 A C<0$. For the sake of completeness, we show our computation of $D$. We have

$$
\begin{aligned}
\frac{Q Q^{\prime} r r^{\prime}}{R R^{\prime} q q^{\prime}}+ & \frac{R R^{\prime} q q^{\prime}}{Q Q^{\prime} r r^{\prime}}=\frac{Q^{2} Q^{\prime 2} r^{2} r^{\prime 2}+R^{2} R^{\prime 2} q^{2} q^{2}}{R Q R^{\prime} Q^{\prime} r q r^{\prime} q^{\prime}} \\
= & \frac{1}{R Q R^{\prime} Q^{\prime} r q r^{\prime} q^{\prime}}\left\{\left(s^{2}+b^{2}\right)\left(s^{2}+b^{\prime 2}\right)\left(t^{2}+a^{2}\right)\left(t^{2}+a^{\prime 2}\right)\right. \\
& \left.+\left(s^{2}+a^{2}\right)\left(s^{2}+a^{\prime 2}\right)\left(t^{2}+b^{2}\right)\left(t^{2}+b^{\prime 2}\right)\right\} \\
= & \frac{1}{R Q R^{\prime} Q^{\prime} r q r^{\prime} q^{\prime}}\left\{2 s^{4} t^{4}+c_{1}\left(s^{4} t^{2}+s^{2} t^{4}\right)+c_{2}\left(s^{4}+t^{4}\right)\right. \\
& \left.+c_{3} s^{2} t^{2}+c_{4}\left(s^{2}+t^{2}\right)+2 a^{2} b^{2} a^{\prime 2} b^{\prime 2}\right\}
\end{aligned}
$$

with

$$
\begin{array}{ll}
c_{1}=a^{2}+b^{2}+a^{\prime 2}+b^{\prime 2}, & c_{2}=a^{2} a^{\prime 2}+b^{2} b^{\prime 2}, \\
c_{3}=2\left(a^{2}+a^{\prime 2}\right)\left(b^{2}+b^{\prime 2}\right), & c_{4}=a^{2} a^{\prime 2}\left(b^{2}+b^{\prime 2}\right)+\left(a^{2}+a^{\prime 2}\right) b^{2} b^{\prime 2} .
\end{array}
$$

We have

$$
\begin{aligned}
2 \cos ( & \left.\alpha+\alpha^{\prime}\right) \cos \left(\beta+\beta^{\prime}\right)+\frac{s^{2}+t^{2}}{s t} \sin \left(\alpha+\alpha^{\prime}\right) \sin \left(\beta+\beta^{\prime}\right) \\
= & 2\left\{\cos \alpha \cos \alpha^{\prime}-\sin \alpha \sin \alpha^{\prime}\right\}\left\{\cos \beta \cos \beta^{\prime}-\sin \beta \sin \beta^{\prime}\right\} \\
& +\frac{s^{2}+t^{2}}{s t}\left\{\sin \alpha \cos \alpha^{\prime}+\cos \alpha \sin \alpha^{\prime}\right\}\left\{\sin \beta \cos \beta^{\prime}+\cos \beta \sin \beta^{\prime}\right\} \\
= & 2\left\{\frac{\left(s^{2}+a b\right)\left(s^{2}+a^{\prime} b^{\prime}\right)}{R Q R^{\prime} Q^{\prime}}-\frac{s^{2}(b-a)\left(b^{\prime}-a^{\prime}\right)}{R Q R^{\prime} Q^{\prime}}\right\} \\
& \times\left\{\frac{\left(t^{2}+a b\right)\left(t^{2}+a^{\prime} b^{\prime}\right)}{r q r^{\prime} q^{\prime}}-\frac{t^{2}(b-a)\left(b^{\prime}-a^{\prime}\right)}{r q r^{\prime} q^{\prime}}\right\} \\
& +\frac{s^{2}+t^{2}}{s t}\left\{\frac{s(b-a)\left(s^{2}+a^{\prime} b^{\prime}\right)}{R Q R^{\prime} Q^{\prime}}+\frac{\left(s^{2}+a b\right) s\left(b^{\prime}-a^{\prime}\right)}{R Q R^{\prime} Q^{\prime}}\right\}
\end{aligned}
$$




$$
\begin{aligned}
& \times\left\{\frac{t(b-a)\left(t^{2}+a^{\prime} b^{\prime}\right)}{r q r^{\prime} q^{\prime}}+\frac{\left(t^{2}+a b\right) t\left(b^{\prime}-a^{\prime}\right)}{r q r^{\prime} q^{\prime}}\right\} \\
= & \frac{1}{R Q R^{\prime} Q^{\prime} r q r^{\prime} q^{\prime}}\left[2\left\{\left(s^{2}+a b\right)\left(s^{2}+a^{\prime} b^{\prime}\right)-s^{2}(b-a)\left(b^{\prime}-a^{\prime}\right)\right\}\right. \\
& \times\left\{\left(t^{2}+a b\right)\left(t^{2}+a^{\prime} b^{\prime}\right)-t^{2}(b-a)\left(b^{\prime}-a^{\prime}\right)\right\} \\
& +\left(s^{2}+t^{2}\right)\left\{(b-a)\left(s^{2}+a^{\prime} b^{\prime}\right)+\left(s^{2}+a b\right)\left(b^{\prime}-a^{\prime}\right)\right\} \\
& \left.\times\left\{(b-a)\left(t^{2}+a^{\prime} b^{\prime}\right)+\left(t^{2}+a b\right)\left(b^{\prime}-a^{\prime}\right)\right\}\right] \\
= & \frac{1}{R Q R^{\prime} Q^{\prime} r q r^{\prime} q^{\prime}}\left\{2 s^{4} t^{4}+d_{1}\left(s^{4} t^{2}+s^{2} t^{4}\right)+d_{2}\left(s^{4}+t^{4}\right)+d_{3} s^{2} t^{2}\right. \\
& \left.+d_{4}\left(s^{2}+t^{2}\right)+2 a^{2} b^{2} a^{\prime 2} b^{\prime 2}\right\}
\end{aligned}
$$

with

$$
\begin{aligned}
& d_{1}=2\left\{a b+a^{\prime} b^{\prime}-(b-a)\left(b^{\prime}-a^{\prime}\right)\right\}+\left(b-a+b^{\prime}-a^{\prime}\right)^{2} \\
& d_{2}=2 a b a^{\prime} b^{\prime}+\left(b-a+b^{\prime}-a^{\prime}\right)\left\{(b-a) a^{\prime} b^{\prime}+a b\left(b^{\prime}-a^{\prime}\right)\right\} \\
& d_{3}=2\left\{a b+a^{\prime} b^{\prime}-(b-a)\left(b^{\prime}-a^{\prime}\right)\right\}^{2} \\
& \quad \quad+2\left(b-a+b^{\prime}-a^{\prime}\right)\left\{(b-a) a^{\prime} b^{\prime}+a b\left(b^{\prime}-a^{\prime}\right)\right\} \\
& d_{4}=2 a b a^{\prime} b^{\prime}\left\{a b+a^{\prime} b^{\prime}-(b-a)\left(b^{\prime}-a^{\prime}\right)\right\}+\left\{(b-a) a^{\prime} b^{\prime}+a b\left(b^{\prime}-a^{\prime}\right)\right\}^{2} .
\end{aligned}
$$

Since

$$
\begin{aligned}
& d_{1}=c_{1}, \quad d_{4}=c_{4}, \quad d_{2}-c_{2}=(b-a)\left(b^{\prime}-a^{\prime}\right)\left(b^{\prime}-a\right)\left(a^{\prime}-b\right), \\
& d_{3}-c_{3}=-2(b-a)\left(b^{\prime}-a^{\prime}\right)\left(b^{\prime}-a\right)\left(a^{\prime}-b\right),
\end{aligned}
$$

we have (61). Next we show $A<0$. Since

$$
0 \leq B^{2}<4 A C=16 r_{z} r_{\xi} s t\left\{\frac{1}{(s+t)^{2}} \sin ^{2} \frac{\theta_{z}-\theta_{\xi}}{2}-\frac{1}{(s-t)^{2}} \sin ^{2} \frac{\theta_{z}+\theta_{\xi}}{2}\right\},
$$

we have

$$
\left|\frac{1}{s-t} \sin \frac{\theta_{z}+\theta_{\zeta}}{2}\right|<\frac{1}{s+t} \sin \frac{\theta_{z}-\theta_{\zeta}}{2} \quad\left(-\pi<\theta_{\zeta}<0<\theta_{z}<\pi\right),
$$

and hence

$$
A=-2 \sqrt{r_{z} r_{\zeta}}\left\{\frac{1}{s+t} \sin \frac{\theta_{z}-\theta_{\zeta}}{2}+\frac{1}{s-t} \sin \frac{\theta_{z}+\theta_{\zeta}}{2}\right\}<0 .
$$

Thus (60) holds, which yields (59) $\left(a<b<0<a^{\prime}<b^{\prime}\right)$. In the above argument, the signs of $a, b, a^{\prime}, b^{\prime}$ are not essential. We have (59) as long as $a<b<a^{\prime}<b^{\prime}$. In the case where $E=\left(-\infty, b^{\prime}\right] \cup[a, b] \cup\left[a^{\prime}, \infty\right]$ $\left(b^{\prime}<a<b<a^{\prime}\right)$, we apply (58) to $\Omega=\left\{\left[-y, b^{\prime}\right] \cup[a, b] \cup\left[a^{\prime}, y\right]\right\}^{c}(y>$ 
$\left.\left|a^{\prime}\right|+\left|b^{\prime}\right|\right)$. Letting $y$ tend to $\infty$, we obtain

$$
D^{2} c\left(x, z, \zeta ; E^{c}\right)=\frac{A^{\prime} x^{2}+B^{\prime} x+C^{\prime}}{2|M(z) M(\zeta) \|(x-z)(x-\zeta)|^{2}},
$$

where $M(\xi)(\xi=z, \zeta), A^{\prime}, B^{\prime}, C^{\prime}$ are defined, in the same manner as above, by

$$
\begin{aligned}
& M(\xi)=\lim _{y \uparrow \infty}\left(\frac{\left(b^{\prime}-\xi\right)(b-\xi)(y-\xi)}{(-y-\xi)(a-\xi)\left(a^{\prime}-\xi\right)}\right)^{1 / 2}, \\
& r_{\xi} e^{i \theta \xi}=-\frac{\left(b^{\prime}-\xi\right)(b-\xi)}{(a-\xi)\left(a^{\prime}-\xi\right)} \quad\left(r_{\xi}>0,\left|\theta_{\xi}\right|<\pi, \xi=z, \zeta\right) .
\end{aligned}
$$

Note that $B^{\prime 2}-4 A^{\prime} C^{\prime}$ is equal to the discriminant with respect to $\left[-a^{\prime},-b\right] \cup\left[-a,-b^{\prime}\right], z=i s, \zeta=-i t$. Thus $B^{\prime 2}-4 A^{\prime} C^{\prime}<0$. Since $A^{\prime} C^{\prime}>0$ and $0<\left(\theta_{z}-\theta_{\zeta}\right) / 2<\pi$, we have, in the same manner as above, $A^{\prime}<0$. Thus (59) holds for $b^{\prime}<a<b<a^{\prime}$. This completes the proof of $D^{2} c(\cdot, \cdot, \cdot ; \Omega) \leq 0$ for doubly-connected domains $\Omega$. Combined with Proposition 20, Theorem 3 follows.

Using Theorem 3, we can study $\gamma(A)+\gamma(B)-\gamma(A \cup B)$ for two continua $A, B$ with a quantitative estimate. Moreover, let $A$ be a union of two continua and let $B$ a continuum such that $A \cap B \neq \emptyset$. Then, in the same manner as in $\S 8$, we have $\gamma(A \cup B) \leq \gamma(A)+\gamma(B)$, by Theorem 3 .

Remark 25. The function $c\left(\infty ; \Omega_{t}\right)$ is not, in general, concave. Take $\Omega=[-1,0]$ and $\Gamma=i y+[0,1](y>0)$, for example. Then Proposition 24 shows that

$$
D c\left(w_{0}, \infty ; \Omega_{0}\right)=\left(\frac{1+y / \sqrt{1+y^{2}}}{2}\right)^{1 / 2}
$$

Choose $y>0$ sufficienty small so that $c\left(\infty ; \Omega_{1}\right) \geq 0.49$ and $D c\left(w_{0}, \infty ; \Omega_{0}\right)$ $\leq 0.7$. Then, if $D c\left(w_{t}, \infty ; \Omega_{t}\right) \leq D c\left(w_{0}, \infty ; \Omega_{0}\right)(0 \leq t \leq 1)$, we have

$$
0.49 \leq c\left(\infty ; \Omega_{1}\right) \leq c\left(\infty ; \Omega_{0}\right)+\frac{1}{4} D c\left(w_{0}, \infty ; \Omega_{0}\right) \leq \frac{1.7}{4} \leq 0.43,
$$

which is a contradiction. Thus $\partial c\left(\infty ; \Omega_{t}\right) / \partial t=D c\left(w_{t}, \infty ; \Omega_{t}\right) / 4$ is not decreasing.

\section{REFERENCES}

[1] L. Ahlfors, Bounded analytic functions, Duke. Math. J., 14 (1947), 1-11.

[2] L. Ahlfors and A. Beurling, Conformal invariants and function-theoretic null sets, Acta Math., 83 (1950), 100-134. 
[ 3 ] W. Baker II, Kernel functions on domains with hyperelliptic double, Trans. Amer. Math. Soc., 231 (1977), 339-347.

[4] S. Bergman, The kernel function and conformal mapping, Math. Surveys V, Amer. Math. Soc., New York, 1950.

[5] A. Davie, Analytic capacity and approximation problems, Trans. Amer. Math. Soc., 171 (1972), 409-444.

[6] J. D. Fay, Theta functions on Riemann surfaces, Lecture Notes in Math. 352, Springer-Verlag, Berlin, 1973.

[ 7 ] T. Gamelin, Uniform Algebras, Chelsea, New York, 1984.

[8] P. Garabedian, Schwarz's lemma and the Szegö kernel function, Trans. Amer. Math. Soc., 67 (1949), 1-35.

[ 9 ] _ - Distortion of length in conformal mapping, Duke Math. J., 16 (1949) , 439459.

[10] P. Garabedian and M. Schiffer, Identities in the theory of conformal mapping, Trans. Amer. Math. Soc., 65 (1949), 187-238.

[11] J. Garnett, Analytic capacity and measure, Lecture Notes in Math. 297, SpringerVerlag, Berlin, 1972.

[12] H. Hancock, Lectures on the theory of elliptic functions, Dover, New York, 1958.

[13] S. Ya. Havinson, Analytic capacity of sets, joint non-triviality of various classes of analytic functions and the Schwarz lemma in arbitrary domains, (Russian) Mat. Sb., 54 (1961), 3-50.

[14] D. A. Hejhal, Theta functions, kernel functions and Abel integrals, Mem. Amer. Math. Soc., 129 (1972).

[15] Y. Komatu, Conformal mapping II, (Japanese) Kyoritsu, Tokyo, 1947.

[16] L. M. Milne-Thomson, Theoretical hydrodynamics, Fifth edition, Macmillan, London, 1968.

[17] T. Murai, A real variable method for the Cauchy transform, and analytic capacity, Lecture Notes in Math. 1307, Springer-Verlag, Berlin, 1988.

[18] - The power $3 / 2$ appearing in the estimate of analytic capacity, Pacific J. Math., 143 (1990), 313-340.

[19] —- Analytic capacity for two segments, Nagoya Math. J., 122 (1991), 19-42.

[20] - A formula for analytic separation capacity, Kōdal Math. J., 13 (1990), 265-288.

[21] Z. Nehari, Conformal mapping, Dover, New York, 1952.

[22] Ch. Pommerenke, Über die analytische Kapazität, Arch. Math., 11 (1960), 270 277.

[23] L. Sario and K. Oikawa, Capacity functions, Springer-Verlag, Berlin, 1969.

[24] M. Schiffer, On various types of orthogonalization, Duke Math. J., 17 (1950), 329366.

[25] - Some recent developments in the theory of conformal mapping; in R. Courant, Dirichlet's principle, 249-318, Pure Appl. Math. III, Interscience, New York, 1967.

[26] M. Schiffer and N. S. Hawley, Connections and conformal mapping, Acta Math., 107 (1962), 175-274.

[27] —_, Half-order differentials on Riemann surfaces, Acta Math., 115 (1966), 199236.

[28] M. Schiffer and D. C. Spencer, Functionals of finite Riemann surfaces, Princeton Univ. Press, New Jersey, 1954.

[29] E. P. Smith, The Garabedian function of an arbitrary compact set, Pacific J. Math., 51 (1974), 289-300.

[30] N. Suita, On a metric induced by analytic capacity, Kōdai Math. Sem. Rep., 25 (1973), 215-218. 
[31] - On a metric induced by analytic capacity II, Kōdai Math. Sem. Rep., 27 (1976), 159-162.

[32] - On subadditivity of analytic capacity for two continua, Kōdai Math. J., 7 (1984), 73-75.

[33] M. Tsuji, Potential theory in modern function theory, Maruzen, Tokyo, 1975.

[34] A. G. Vitushkin, Analytic capacity of sets in problems of approximation theory, (Russian) Uspehi Mat. Nauk, 22 (1967), 141-199.

[35] L. Zalcman, Analytic capacity and rational approximation, Lecture Notes in Math. 50, Springer-Verlag, Berlin, 1968.

Department of Mathematics

School of Science

Nagoya University

Chikusa-ku, Nagoya, 464-01

Japan 